\title{
ARRANGEMENTS OF AN $M$-QUINTIC WITH RESPECT TO A CONIC THAT MAXIMALLY INTERSECTS ITS ODD BRANCH
}

\author{
S. YU. OREVKOV
}

\begin{abstract}
Under certain assumptions, the arrangements mentioned in the title are classified up to isotopy. Their algebraic realizability is discussed.
\end{abstract}

\section{$\S 0$. INTRODUCTION}

0.1. Statement of main results. The connected components of the set of real points of a plane projective real curve are called branches. A branch is even (or an oval) if it is zero-homologous in $\mathbb{R}^{2}$. Otherwise it is odd (or a pseudoline).

Theorem 1. a) Let $\mathcal{J}$ be a tame almost complex structure in $\mathbb{C P}^{2}$ that is invariant under complex conjugation, and let $C_{5}$ and $C_{2}$ be nonsingular real $\mathcal{J}$-holomorphic $M$-curves in $\mathbb{R P}^{2}$ of degrees 5 and 2 , respectively. Let $J_{5}$ be the odd branch of $C_{5}$. Suppose that $J_{5}$ intersects $C_{2}$ at ten distinct real points. Then the arrangement of $C_{5} \cup C_{2}$ in $\mathbb{R P}^{2}$ is one of those listed in Subsection 0.5 up to isotopy. All these arrangements are realizable.

b) All the arrangements except the six of them labeled by “\#* alg." or " $¥$ alg." are realizable by real algebraic curves of degrees 5 and 2 .

c) The two arrangements labeled by "\# alg." are unrealizable by real algebraic curves of degrees 5 and 2.

Theorem 2. a) Let $\mathcal{J}$ be a tame almost complex structure in $\mathbb{C P}^{2}$ that is invariant under complex conjugation, and let $C_{5}, L_{1}$, and $L_{2}$ be nonsingular real $\mathcal{J}$-holomorphic $M$-curves in $\mathbb{R P}^{2}$ of degrees 5,1 , and 1 , respectively. Suppose that the odd branch $J_{5}$ of $C_{5}$ intersects each of the lines $L_{1}$ and $L_{2}$ at five distinct real points. Then either the arrangement of $C_{5} \cup L_{1} \cup L_{2}$ in $\mathbb{R P}^{2}$ is one of those listed in Subsections 0.6, 0.7 and in Figures 16.1-16.22, or $C_{5} \cup L_{1} \cup L_{2}$ realizes one of the sixteen arrangements such that $L_{1}=\{x=0\}$ and $L_{2}=\{x+\varepsilon y=0\}$, where $L_{1}$ is a line intersecting $J_{5}$ at five points and $0<\varepsilon \ll 1$.

All these arrangements are realizable.

b) All of them are algebraically realizable except the arrangement in Figure 16.12 and the five arrangements in Subsection 0.7 labeled by “尹* alg." or “尹 alg.".

c) The three arrangements in Subsection 0.7 labeled by "\# alg." and that in Figure 16.12 are unrealizable by real algebraic curves.

Theorems 1 and 2 are proved in $\S \S 2-7$. A general outline of the proof is given in Subsections 0.2 and 0.4 .

Remark 1. I know a proof of the algebraic unrealizability of the four arrangements in Subsection 0.5 and of the two in Subsection 0.7 that are labeled by " $\nexists^{*}$ alg.", but this paper is so long that I decided not to include it. Maybe, I shall write it somewhere else.

2000 Mathematics Subject Classification. Primary 57R52, 57R19.

Key words and phrases. Plane projective real curve, almost complex structure, isotopy, algebraic (un)realizability. 
Remark 2. An essential part of the classification described by Theorem 2 was obtained earlier in [9, 10] (see more details at the end of Subsection 0.4). However, it happens that most of the unrealizability statements (restrictions) proved there can be obtained "for free" as corollaries to other results of the present paper (see Subsection 7.1). So, for the reader's convenience, I added Subsection 7.2 (only half a page long) where I give another proof of the remaining restrictions from 9, 10, making the restriction part of Theorems 1 and 2 self-contained. To the contrary, here I give proofs of realizability (constructions) only when they were not given in [9, 10].

A particular case of Theorem 1 admits the following generalization to an arbitrary degree. This statement is used essentially in the proof of Theorems 1 and 2 (see Subsection 6.5). Let $O$ be a simple closed curve in $\mathbb{R P}^{2}$. It splits $\mathbb{R P}^{2}$ into a disk $D$ and a Möbius band $M$. We say that $n$ pairwise disjoint smoothly embedded segments $I_{1}, \ldots, I_{n}$ with endpoints on $O$ form a nest of depth $n$ inside $O$ (respectively, outside $O$ ) if there exist disks

$$
D_{1} \subset D_{2} \subset \cdots \subset D_{n} \subset \mathbb{R P}^{2}
$$

such that $I_{j}=D \cap\left(\partial D_{j}\right)$ (respectively, $\left.I_{j}=M \cap\left(\partial D_{j}\right)\right)$.

Theorem 3. Let $C_{2}$ and $C_{n}$ be real pseudoholomorphic (for example, real algebraic) nonsingular $M$-curves in $\mathbb{R P}^{2}$ of degrees 2 and $n$, respectively. Suppose that $C_{2}$ intersects a branch $B_{n}$ of $C_{n}$ at $2 n$ distinct real points and that all other branches of $C_{n}$ are contained in the exterior of $C_{2}$. Suppose that $B_{n}$ has the parity of $n$ (i.e., either $n$ is even, or $n$ is odd and then $B_{n}$ is the odd branch of $\left.C_{n}\right)$. Then there exists a nest of depth $n$ inside $C_{2}$ formed by arcs of $B_{n}$.

This result is proved in $\S 1$. The proof is based on the complex orientations formula for curves on a 2-sphere and on a generalization for quadrics of Fiedler's theorem [3 on symmetric $M$-curves. The rest of the introduction is not needed for its understanding.

Remark 3. The following question arises from comparing Theorem 3 with the classification of arrangements of an $M$-quintic with respect to a conic that maximally intersects one of its ovals 14 .

Suppose that $B_{n}$ and $n$ have different parities (i.e., $n$ is odd and $B_{n}$ is an oval of $C_{n}$ ) but all the other conditions of Theorem 3 are satisfied. Does this imply that there exists a nest of depth $n-1$ inside $C_{2}$ formed by arcs of $B_{n}$ ?

The proof of Theorem 3 given below does not extend to this case for the following reason. The lift of the odd branch of $C_{n}$ to the double covering branched along $C_{2}$ is connected, so that the lift of $C_{n}$ is an $(M-1)$-curve rather than an $M$-curve on the hyperboloid, and the rest of the proof fails. On the other hand, this is an additional informal argument for the plausibility of the affirmative answer, because many properties of $(M-1)$-curves "differ by one" (whatever it means) from the corresponding properties of $M$-curves.

I am grateful to G.M. Polotovskiu for useful discussions and information about the main results of the paper [10] before its writing had been finished.

0.2. Arrangements realizable pseudoholomorphically but not algebraically. As is seen in the statements of Theorems 1 and 2, some arrangements (those labeled by " $\nexists$ alg." in Subsections 0.5, 0.7 and that in Figure 16.12) contribute to the collection of known examples of isotopy types that are realizable by real pseudoholomorphic curves but unrealizable by real algebraic curves. 
The algebraic unrealizability of two of them (in Figures 21.1 and 16.12) is proved in Subsections 4.1 and 4.4, respectively. The remaining cases are reduced to these two. Namely, the unrealizability of Figure 21.2 and Figure 21.3 reduces to that of Figure 16.12 and of Figure 21.1; see Subsection 4.2 and Subsection 4.3, respectively. The unrealizability of the two arrangements of a quintic and a conic labeled by " $\nexists$ alg." reduces to the corresponding arrangements of a quintic and a pair of lines in accordance with Corollary 5.2 .

The proof of the algebraic unrealizability of Figure 21.1 repeats almost word-forword the end of the proof of the nonexistence of any real algebraic affine sextic of type $C_{2}(1,3,6)$ in [22, §3.4]. However, the proof for Figure 16.12 in Subsection 4.4 involves a new approach, more precisely, a new combination of two old ones: 1) consideration of the cubic resolvent $R$ of a curve of bidegree $(4, n)$ on a ruled surface and its arrangement with respect to the core $L$ (this idea was already used by the author in [18, $\S 6]$ and in 22 , $\S 3]) ; 2$ ) application of the Burau representation of the groupoid of colored braids to the braid corresponding to $R \cup L$ (this idea was used in [15]). In Remark 4.7, a more detailed description (compared to [15]) is given for the Burau representation of the groupoid of colored braids. In my opinion, it is more convenient for practical computations.

In Appendix A, this approach is applied in order to obtain a new "and a more reliable" proof of one of the main results of [21] (see the discussion after Proposition A.1).

In Appendix C, we apply the method of cubic resolvents to prove the algebraic unrealizability of some (not fiberwise) isotopy types of curves on the ruled surface $\mathcal{F}_{4}$. Moreover, these isotopy types are pseudoholomorphically realizable in a tame almost complex structure with the exceptional curve.

0.3. Zigzag removal. All the pseudoholomorphic unrealizability statements not provided by Theorem 3 are proved in this paper by using the method proposed in [13, 16, i.e., by reducing the question about the realizability of a curve to the question about the quasipositivity of some braids (but the collection of quasipositivity criterions is enriched here by a result of Florens [5]; see Theorem 6.6).

The braids are determined by the arrangements of the curve with respect to a pencil of lines (fiberwise arrangements). To exclude a given isotopy type, one should treat all corresponding fiberwise arrangements. The number of cases can be reduced considerably by using the fact that the unrealizability of some fiberwise arrangements follows from the unrealizability of the arrangements obtained from them by certain elementary moves (for example, by rectifying S-like zigzags).

In all previous papers where this method was used by myself or by other authors, the reduction of this sort of all fiberwise arrangements to some restricted subset of them was assumed evident, and the details (consisting in routine case-by-case considerations) were omitted. Usually, it was really evident, but in the case of a quintic and a conic (as well as of a quartic and a cubic) the number of cases to be considered becomes too large.

One very particular arrangement of a quintic and a conic was claimed to be excluded in [13, Theorem 1.2A], as an example of an application of the method of braids. However, this statement is wrong and the mistake appeared because I missed one possibility for the fiberwise arrangement, the one that is realizable (this mistake was discussed in [9]). Some other similar restrictions were formulated in [7, but most of them are erroneous for the same reason.

To avoid repetition of this kind of error, some rigorous statements about reduction of fiberwise arrangements to others are formulated and proved in Subsections 6.3 and 6.4.

Note that the statement on removing S-like zigzags is valid only for pseudoholomorphic curves. An example given in Appendix B shows that, in general, it is wrong for algebraic curves. 
0.4. Types and series. General outline of proofs of Theorems 1 and 2. Following Polotovskiı, we divide all arrangements mentioned in Theorems 1 and 2 into eight types $(8=3+5)$ as described below.

Let $C_{5}, J_{5}$, and $C_{2}$ be as in Theorem 1 . Let $\Gamma_{\infty}$ be a pseudoline (i.e., an embedded circle not contractible in $\mathbb{R P}^{2}$ ) disjoint from $C_{2}$ and intersecting $J_{5}$ at the minimal possible number $n$ of points. Then the intersection points are called passages through infinity, and $n$ is called the type of the arrangement of $C_{5}$ with respect to $C_{2}$. It is clear that $n$ can only take the values 1,3 , or 5 .

Let $C_{5}, J_{5}, L_{1}$, and $L_{2}$ be as in Theorem 2. The lines $L_{1}$ and $L_{2}$ split $\mathbb{R P}^{2}$ into two domains. We denote them by $D_{1}$ and $D_{2}$. The lines cut $J_{5}$ into ten arcs. Let $d_{j}$, $j=1,2$, be the number of the arcs lying in $D_{j}$ and connecting one line to the other. The unordered pair $\left(d_{1}, d_{2}\right)$ is called the type of the arrangement of $C_{5}$ with respect to $L_{1}$ and $L_{2}$. It is clear that each of $d_{1}, d_{2}$ can only take the values 1,3 , and 5 , the type $(5,5)$ being impossible. Thus, all the arrangements are divided naturally into five types: $(1,1),(1,3),(3,3),(1,5)$, and $(3,5)$.

We shall subdivide each type into series in accordance with the isotopy types of the arrangements of $J_{5}$ with respect to $C_{2}$ or $L_{1} \cup L_{2}$. In the lists in Subsections 0.5-0.7 and throughout the paper, the series are referred to by numbers typed in boldface.

Clearly, the objects described by Theorems 1 and 2 are very close to each other. Indeed, when a pair of lines is perturbed into a nonsingular conic, a curve $C_{5} \cup L_{1} \cup L_{2}$ satisfying the hypothesis of Theorem 2 is transformed into a curve $C_{5} \cup C_{2}$ satisfying the hypothesis of Theorem 1. Moreover, in many cases (see $\S 5$ ) a curve $C_{5} \cup C_{2}$ satisfying the hypothesis of Theorem 1 can degenerate into a curve satisfying the hypothesis of Theorem 2. For this reason, many cases of the classification of curves of the form $C_{5} \cup C_{2}$ may be reduced to the classification of $C_{5} \cup L_{1} \cup L_{2}$ and vice versa. This concerns equally the algebraic and the pseudoholomorphic curves.

In constructions we use only perturbations $L_{1} \cup L_{2} \rightarrow C_{2}$. However, in restrictions we use such reductions both ways. Therefore, to eliminate the reader's possible suspicion about a vicious circle, we present the main steps of the proof of the restrictions in the pseudoholomorphic case (the algebraic case was discussed in Subsection 0.2).

Step 1. In the following cases, both algebraic and pseudoholomorphic classifications are reduced to classification of the arrangements of $C_{5} \cup C_{1}$, i.e., to the case of a quintic and a single line intersecting maximally:

(1.1) the arrangements of $C_{5} \cup L_{1} \cup L_{2}$ of the types $(1,5)$ and $(3,5)$;

(1.2) the arrangements of $C_{5} \cup C_{2}$ with five passages through infinity;

(1.3) the arrangements of $C_{5} \cup C_{2}$ with one or three passages through infinity that have five nested arcs inside $C_{2}$ (see the definition in Subsection 0.1).

Originally, the classification of $C_{5} \cup C_{1}$ was obtained by Polotovskiu in 23], by using methods working only for algebraic curves. However, it can also be derived from the Kharlamov-Viro congruence [8] (which has been found partially due to this classification). This congruence applies to the pseudoholomorphic curves as well.

Step 2 (§3). Complete classification of the arrangements of $C_{5} \cup L_{1} \cup L_{2}$ of the type

Step $3(\S 6)$. Restrictions for the arrangements of $C_{5} \cup C_{2}$ with one passage through infinity of the series listed in Subsection 6.1.

Step 4 (Subsection 7.1). Restrictions for the arrangements of $C_{5} \cup L_{1} \cup L_{2}$ of the types $(1,1)$ and $(1,3)$, which are reduced to the restrictions for $C_{5} \cup C_{2}$ proved at Steps 1.2, 1.3 , and 3 . 
Step 5 (Subsection 7.2 or a reference to [9, 10]). Restrictions for the arrangements of $C_{5} \cup L_{1} \cup L_{2}$ of the types $(1,1)$ and $(1,3)$ that were not proved at Step 4. This completes the proof of Theorem 2 .

Step 6. By Corollary 5.2, all the remaining restrictions for the arrangements of $C_{5} \cup C_{2}$ (i.e., for the series with one passage through infinity that were not considered at Step 3 and for all arrangements with three passages through infinity) are reduced to Theorem 2.

In the following table, we present the distribution of the realizable arrangements over the types (in accordance with Remark 1 in Subsection 0.1, the last two lines may be summed).

\begin{tabular}{|c|c|c|c|c|c|c|c|c|}
\hline \multirow[b]{2}{*}{ Type of arrangement } & \multicolumn{4}{|c|}{$\overline{C_{5} \cup C_{2}}$} & \multicolumn{4}{|c|}{ 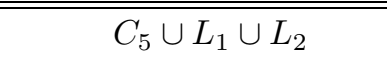 } \\
\hline & 1 & 3 & 5 & $(1,1)$ & $(1,3)$ & $(3,3)$ & $(1,5)$ & $(3,5)$ \\
\hline number of series & 33 & 15 & 2 & 8 & 19 & 7 & 3 & 1 \\
\hline number of ps-hol. arr. & 124 & 48 & 4 & 20 & 56 & 22 & 12 & 4 \\
\hline number of “尹 alg." & - & 2 & - & - & 3 & 1 & - & - \\
\hline number of “尹*alg." & 3 & 1 & - & - & 2 & - & - & - \\
\hline
\end{tabular}

A complete classification of the arrangements of $C_{5} \cup L_{1} \cup L_{2}$ for the type $(1,1)$ and an almost complete classification (except about 15 cases) for the type $(1,3)$ were obtained in [9, 10. Almost all proofs of restrictions in 9, 10] extend automatically to the case of pseudoholomorphic curves (if we take into account the remark at Step 1 concerning the classification of $C_{5} \cup C_{1}$ ). The only exception is the arrangement denoted in [9] by $D 4$. Its unrealizability was derived in 9 ] from the algebraic unrealizability of affine sextics of the isotopy type $B_{2}(1,4,5)$, proved in $[4$. This argument cannot work in the pseudoholomorphic case for the simple reason that such an affine pseudoholomorphic sextic was constructed in 13] (see also [4). All proofs in 9 become valid in the pseudoholomorphic case if the following line is added to Table 2 in the paper [9]:

$$
D 4 \quad \text { - - } \quad X_{2} X_{3} X_{4} \supset_{3} \zeta_{3} \varepsilon_{4} X_{3} X_{2} X_{2} X_{2} X_{2} X_{2} \varepsilon_{3} \subset_{4} X_{5} X_{5} \quad 6 \quad 2 ; 4
$$

0.5. The list of all pseudoholomorphically realizable arrangements of an $M$ quintic and a nonsingular conic maximally intersecting the odd branch.

0.5.1. Encoding of the series. The list is organized by the same rules as the list in [18, §5]. To identify a series (the isotopy type of a mutual arrangement of the intersecting branches), we shall use the encoding proposed by Polotovskil. Namely, we enumerate the points of $J_{5} \cap C_{2}$ by $0,1, \ldots, 9$ in their order along $C_{2}$, so that the point 0 is the endpoint of a component of $J_{5} \backslash C_{2}$ crossing $\Gamma_{\infty}$ and the point 1 is not $\left(\Gamma_{\infty}\right.$ means the same as at the beginning of Subsection 0.4). The isotopy type of $J_{5} \cup C_{2}$ will be encoded by a word $i_{0} i_{1} \ldots i_{9}$ composed of the digits $0, \ldots, 9$ appearing in the same order as the corresponding points appear on $J_{5}$. Moreover, the $\operatorname{arc}\left(i_{9}, i_{0}\right)$ of the quintic must pass through infinity (i.e., it must intersect $\Gamma_{\infty}$ ). Among all words encoding the same isotopy type (if there are no symmetries, the number of them is twice the number of the passages through infinity), we shall always choose the word minimal with respect to the lexicographic order. For the reader's convenience, we shall denote the passages through infinity by the slash "/".

First, we list the arrangements with one passage through infinity, then those with three passages, and then those with five passages. Series of the same type are ordered lexicographically (ignoring the symbol "/"). Arrangements of the same series are placed in a random order. The points $0, \ldots, 9$ are not labeled in pictures, but we mean that they 
are placed on $C_{2}$ clockwise, the point " 0 " being always the uppermost. The numbers written in the pictures denote the number of ovals of the quintic in the corresponding regions.

0.5.2. Encoding of the constructions. Under each arrangement, a reference to its construction(s) is given. This is either the number of a figure with a singular curve to be perturbed (an expression of the form 16.2,7 being an abbreviation for 16.2, 16.7), or one of the following.

$5+1$. This means that $C_{2}=\left\{c_{1}^{2}=\varepsilon f\right\}$, where $\left\{c_{1}=0\right\}$ is a line intersecting $J_{5}$ at five distinct points and $0<\varepsilon \ll 1$.

$m_{n}$. This means that $C_{2}$ is a perturbation of $L_{1} \cup L_{2}$ in the $n$th arrangement of $C_{5} \cup L_{1} \cup L_{2}$ of the type $(1,3)$ in Subsection 0.7 .

References of the form A1, B5, etc. mean that $C_{2}$ is a perturbation of $L_{1} \cup L_{2}$ in the corresponding arrangement of $C_{5} \cup L_{1} \cup L_{2}$ of the type $(1,1)$ constructed in [9] (see also Subsection 0.6).

\subsubsection{Arrangements of $C_{5} \cup C_{2}$ with one passage through infinity.}

1. 0123456789

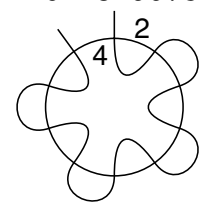

$\mathrm{A} 15,5.2,9.3$

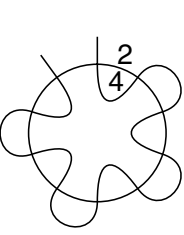

5.1

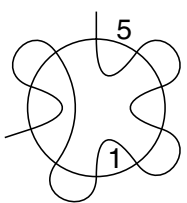

D14

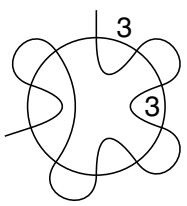

$15_{5}, 9.5$

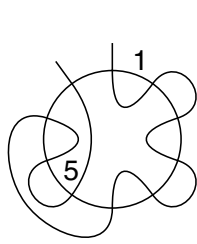

5.1

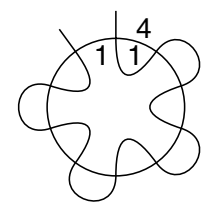

A12, 2.1

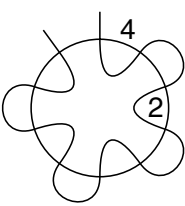

$5.3,9.1$

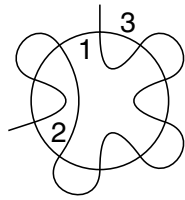

D3, 9.3

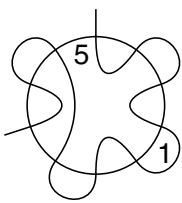

$15_{6}$

4. 0123476589

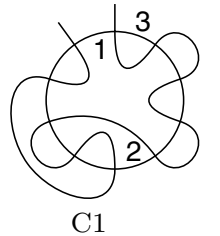

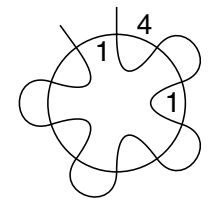

A11, 9.3

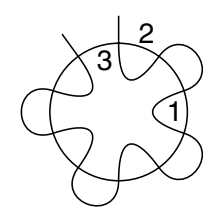

$2.2,9.1,9.2$

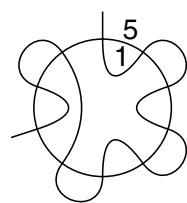

D7, 2.1, 7.2

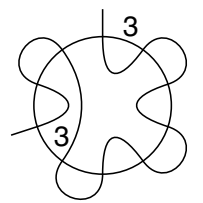

9.1

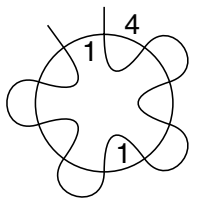

A13

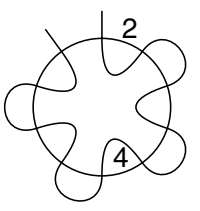

A10

2. 0123456987

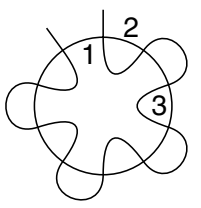

9.2

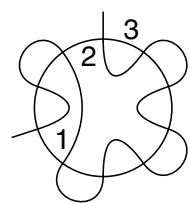

$15_{1}, 2.2,9.1,9.215_{2,3}$

3. 0123458769

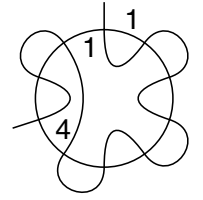

9.2
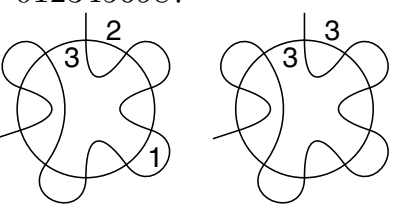

D11

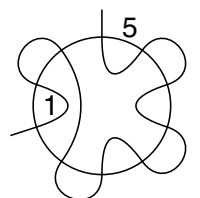

D13, 5.2, 5.3, 9.3

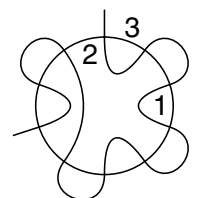

$15_{4}, 9.5$

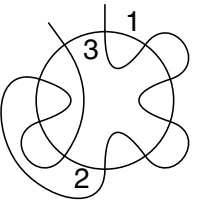

B1

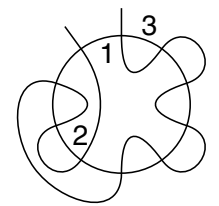

B1, 2.1

5. 0123478965
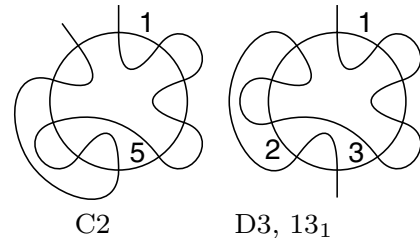

D3, 131
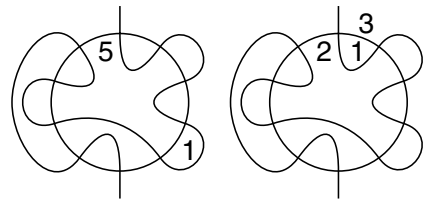

D7, 133

D11, 134 
6. 0123496785

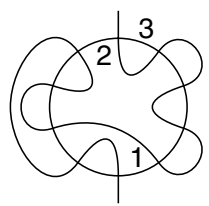

D13, $13_{2}$

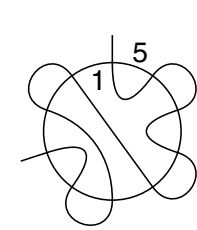

C5

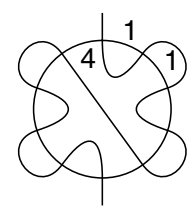

A 11,181

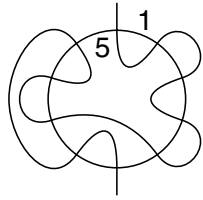

D14, 5.2

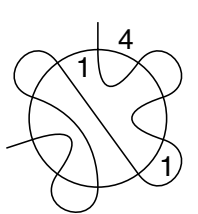

$4_{1}$

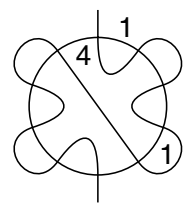

$\mathrm{A} 12,18_{2}$

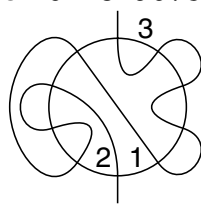

B1, $11_{3}$

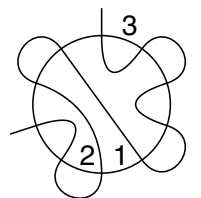

$4_{2}, 9.8$

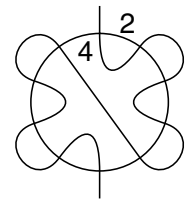

A13, 5.2
9. 0123654789

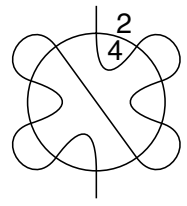

$18_{5}$ (\#* alg.)

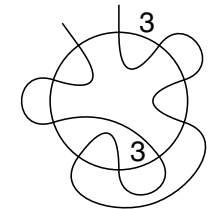

$5.3,9.4$

11. 0123678549

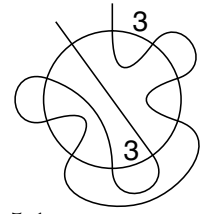

5.1

14. 0125678943

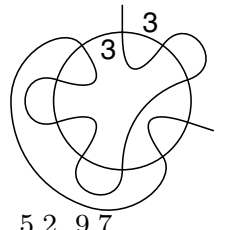

17. 0127654389

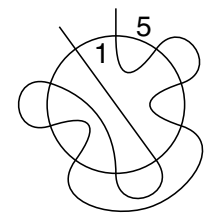

2.1

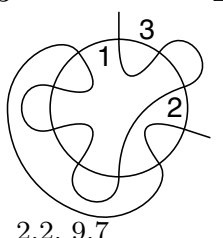

15. 0125698743

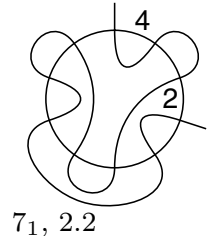

18. 0127896345
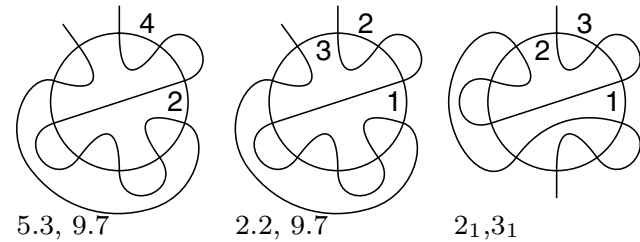

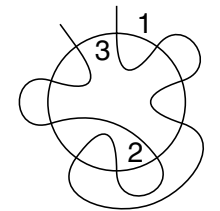

$2.2,9.4$

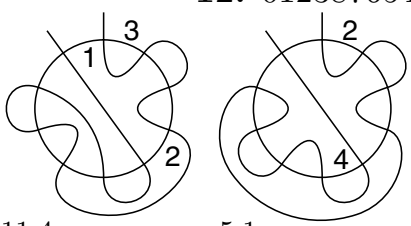

5.1

$17_{1}$

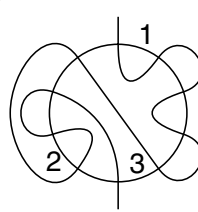

B2, $11_{2}$

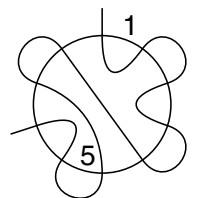

$4_{3}, 9.8$

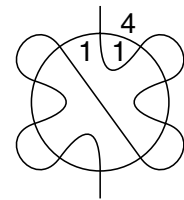

A 14, 184
7. 0123498567

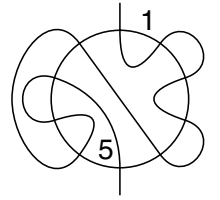

$11_{1}\left(\nexists^{*}\right.$ alg.)

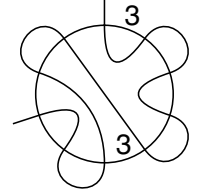

C2, 5.3

8. 0123498765

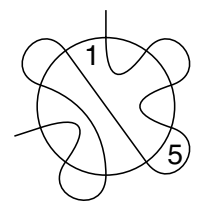

44

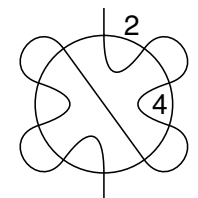

$19_{2}, 9.8$

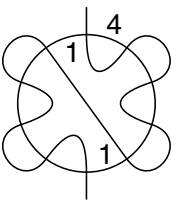

A10, A15, 183

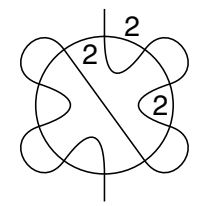

$19_{1}, 9.8$

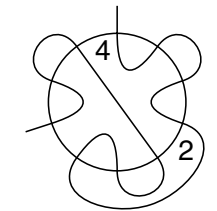

12. 0123876549

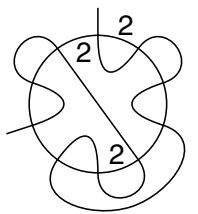

$17_{2}, 2.2,9.4$

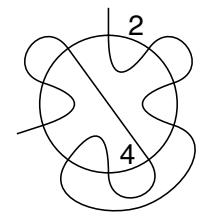

5.2, 9.4

13. 0125436987

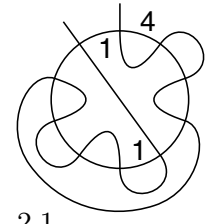

2.1

16. 0127634589

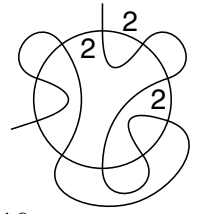

$16_{1}$
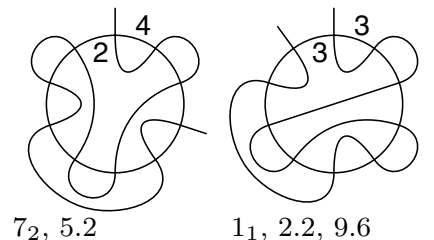

$1_{1}, 2.2,9.6$

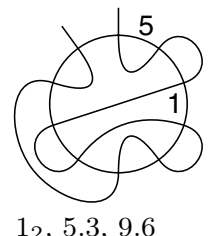

19. 0129456783
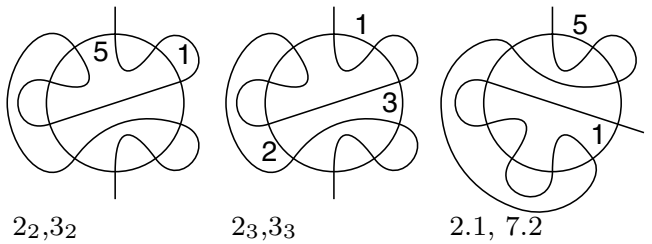
20. 0129654783

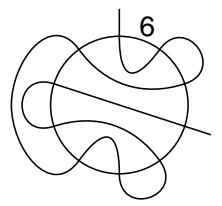

$5+1$

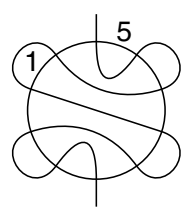

$5+1$

25. 0129876345

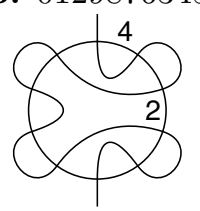

G5, 5.3

26. 0143276589

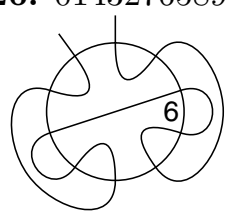

H5

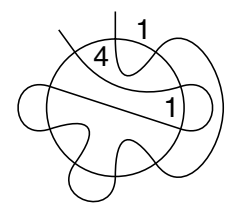

$8_{2}, 7.1$

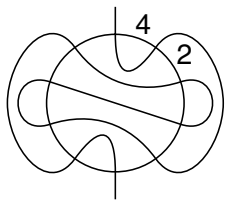

$5+1$

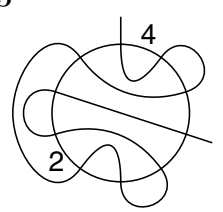

$5+1$

$5+1$

$5+1$

1. 0129834765
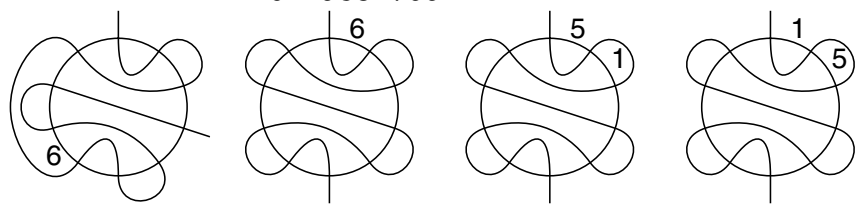

22. 012985436723.0129856743 24. 0129874563
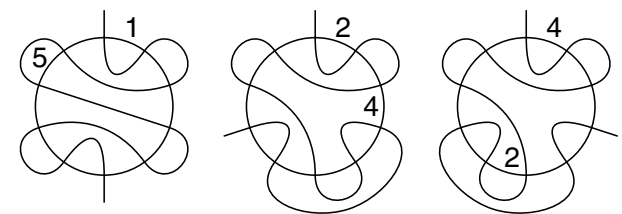

F2, 5.3
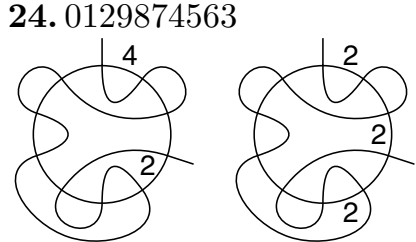

$\mathrm{E} 2,2.1,7.2 \quad \mathrm{E} 4$

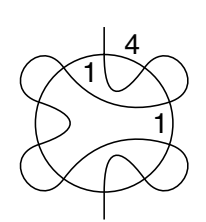

$6_{1,4}, 9.5 \quad 62,3$

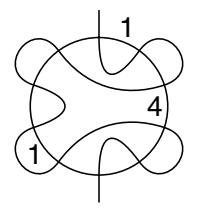

28. 0143296785

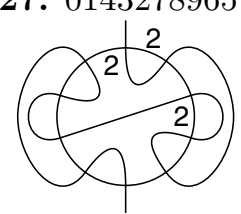

G5, $14_{1}$

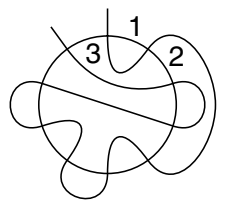

83 ( $\nexists^{*}$ alg.)

32. 0167854329

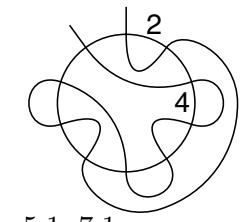

$5.1,7.1$

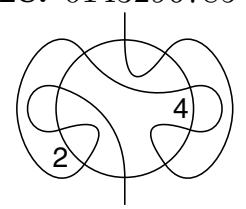

$\mathrm{E} 2,122_{1}$

30. 0145876329

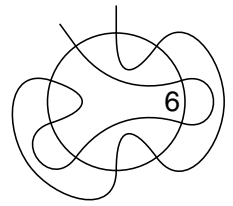

$9_{1}, 5.1,7.1$

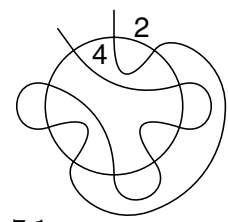

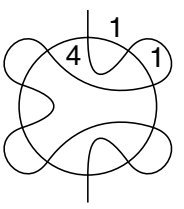

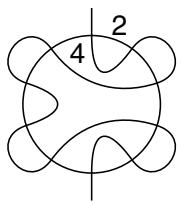

$66,9.5$

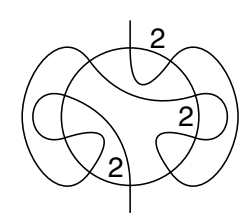

E4, $12_{2}$

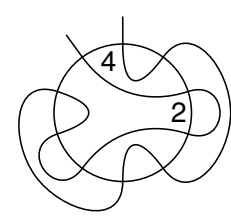

$9_{2}, 7.1$

33. 0187654329

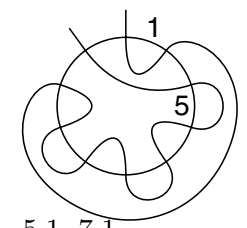

$5.1,7.1$
29. 0145678329

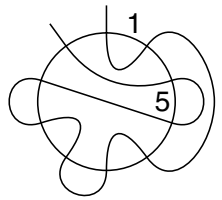

$81,5.1,7.1$

31. 0147832965

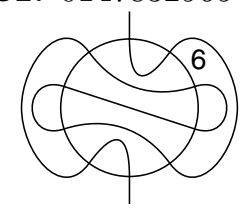

$5+1$

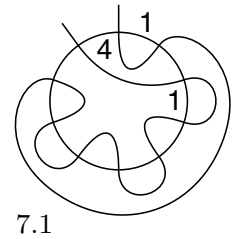

0.5.4. Arrangements of $C_{5} \cup C_{2}$ with three passages through infinity.

34. $012345 / 89 / 67$

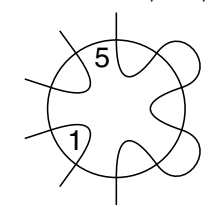

$5_{2}$
35. 012349/65/87
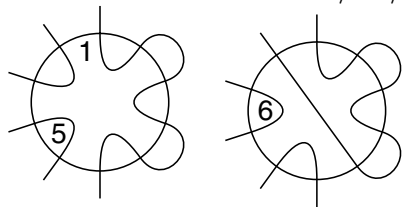

16.19

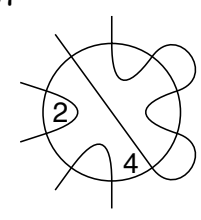

16.20

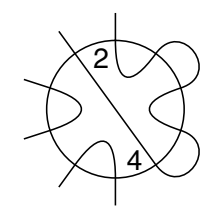

16.21

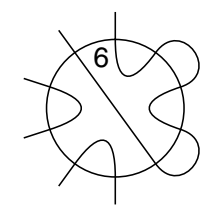

16.22 
36. $0123 / 67 / 4985$

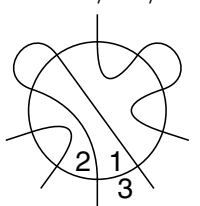

$16.12,8_{3}$ ( $\nexists$ alg.)

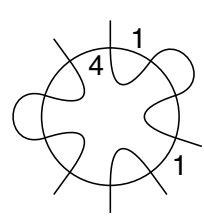

64,183

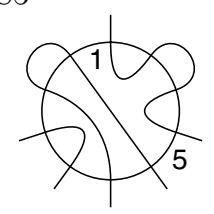

$8_{1}, 16.13$

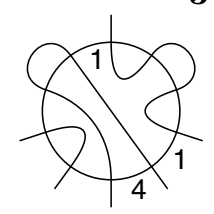

$8_{2}, 16.14$
37.0123/6789/45

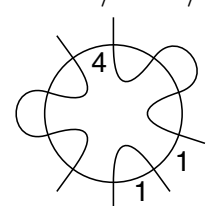

$6_{1}, 18_{4}$
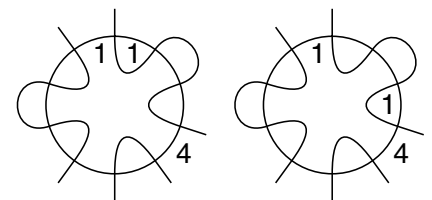

$6_{2,6}, 18_{1}$

$6_{3,5}, 18_{2}$

38. $0123 / 6987 / 45$

39. $0123 / 8769 / 45$
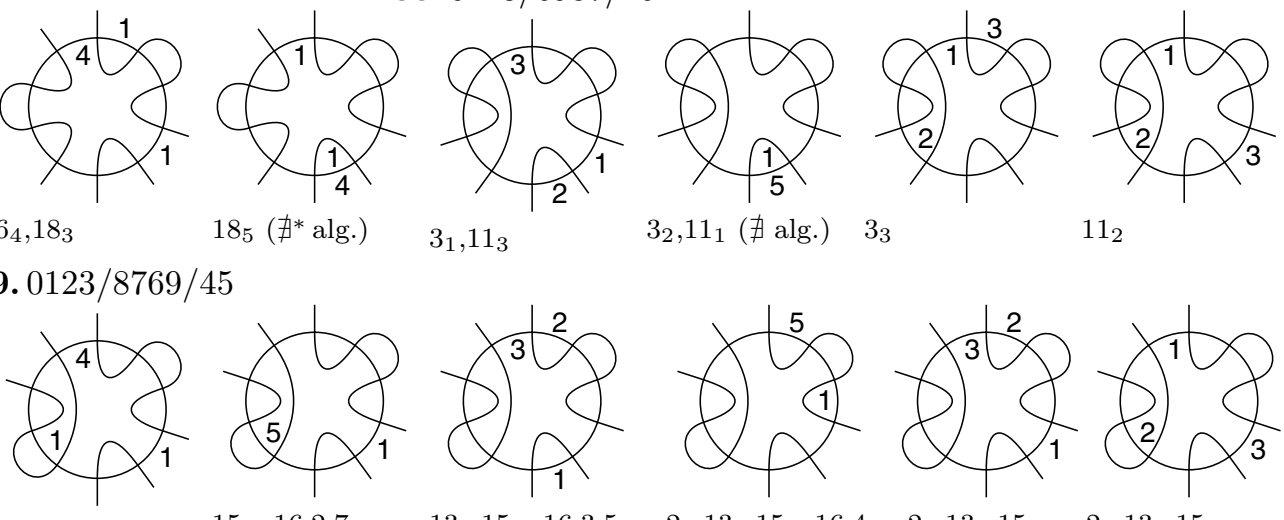

185 (\#* alg.)

$3_{1}, 11_{3}$

$3_{2}, 11_{1}$ (尹 alg.) $\quad 33$

$11_{2}$
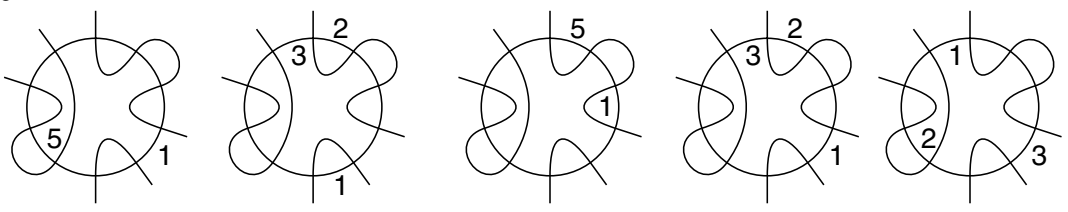

$15_{2}, 16.1,6$

$15_{3}, 16.2,7$

$13_{4}, 15_{1}, 16.3,5$

$2_{2}, 13_{3}, 15_{6}, 16$.

40. $012543 / 89 / 67$

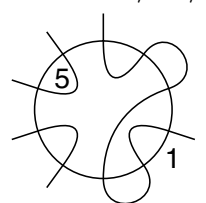

$4_{4}, 16.15$

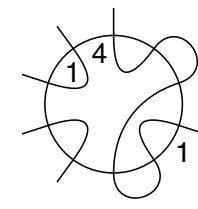

$41,16.16$

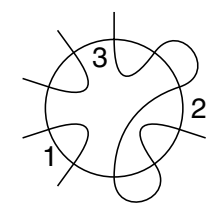

$4_{2}, 16.17$

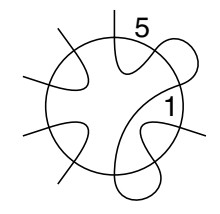

$43,16.18$

41. 012569/43/87

42. $012763 / 89 / 45$

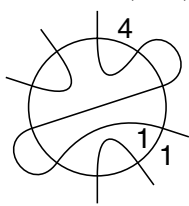

$16.1,16$

44. $0129 / 43 / 8567$

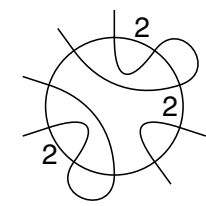

$14_{1}, 16_{1}$

$12_{1}$

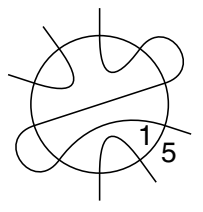

$16.2,15$

$1_{1}, 16.3,17$
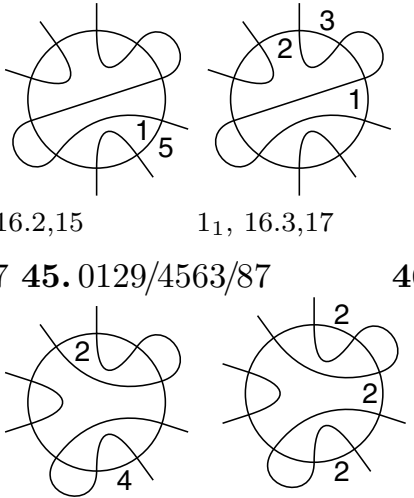

$12_{2}$

47. 012983/67/45

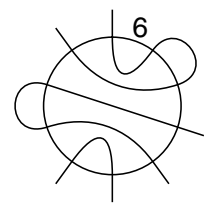

$9_{1}, 16.22$

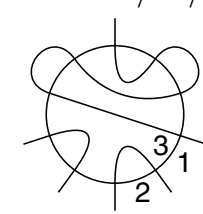

$16.5,12$

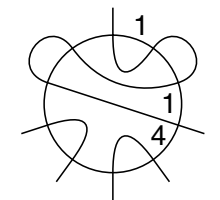

$16.6,14$

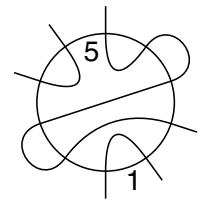

$1_{2}, 16.4,18$
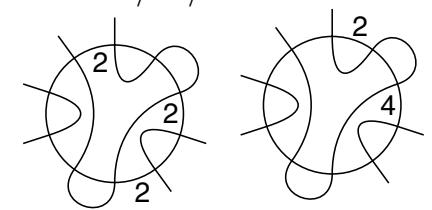

$7_{1}, 16.9,10 \quad 7_{2}, 16.8,11$

43. $012789 / 43 / 65$

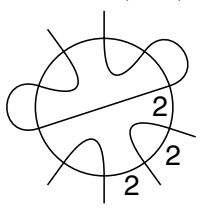

$19_{1}, 16.10$

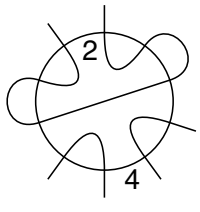

$19_{2}, 16.11$

46. $0129 / 4783 / 65$

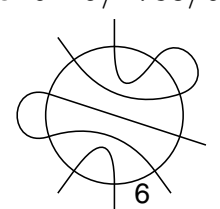

16.19

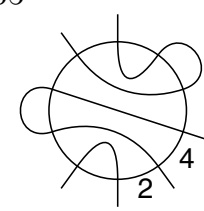

16.20

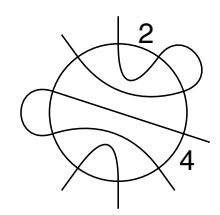

$9_{2}, 16.21$

48. $01 / 45 / 298763$

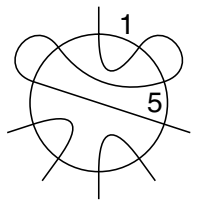

$16.7,13$

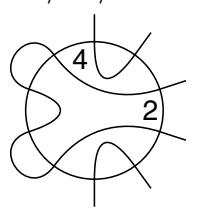

$10_{1}$

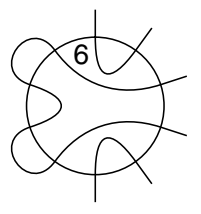

$10_{2}$ 
0.5.5. Arrangements of $C_{5} \cup C_{2}$ with five passages through infinity.

49. $01 / 63 / 87 / 29 / 45$

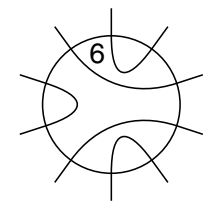

$5+1$

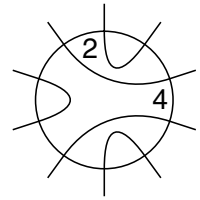

$5+1$
50. $01 / 67 / 23 / 89 / 45$

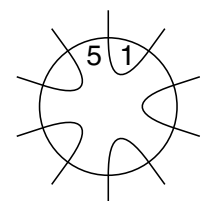

$5+1$

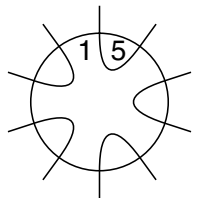

$5+1$

0.6. Pseudoholomorphically realizable arrangements of an $M$-quintic and two lines of the type $(1,1)$. Here we reproduce the list (taken from [9]) of realizable arrangements of $C_{5} \cup L_{1} \cup L_{2}$ of the type $(1,1)$. The list is organized nearly as in Subsection 0.5. The encoding of the arrangements of intersecting branches is as follows (as in Subsection 0.5, it was introduced by Polotovskiı).

We denote the points of $L_{1} \cap J_{5}$ by $0,1,2,3,4$, and the points of $L_{2} \cap J_{5}$ by $5,6,7,8,9$, in the order in which they appear on $L_{1} \backslash\{p\}$ and on $L_{2} \backslash\{p\}$, respectively (here $\{p\}=L_{1} \cap L_{2}$ ). Let $D_{2}$ be the connected component of $\mathbb{R} \mathbb{P}^{2} \backslash\left(L_{1} \cup L_{2}\right)$ on whose boundary the points $0,1, \ldots, 9$ appear in this order, and let $D_{1}$ be the other component. A series is encoded by a word $i_{0} \ldots i_{9}$ composed of the digits $0, \ldots, 9$ that are ordered in the same way as the corresponding points lying on $J_{5}$ and so that the points $i_{0}$ and $i_{9}$ are on different lines and the arc $\left(i_{0}, i_{9}\right)$ of $J_{5}$ is contained in $D_{1}$. As in Subsection 0.5, among all words we choose the lexicographically minimal one. The lines $L_{1}$ and $L_{2}$ are depicted horizontally

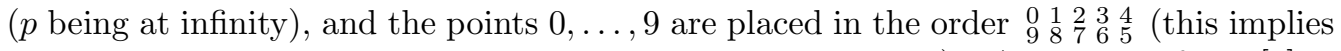
that $D_{2}$ is represented in the pictures by a horizontal band). A notation from [9] is written under each arrangement.

1. 0123456789

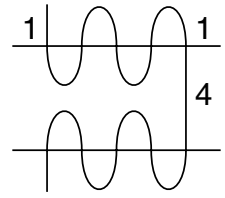

A10

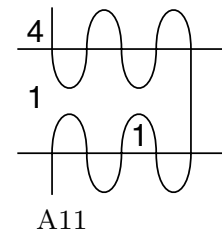

2. 0123456987

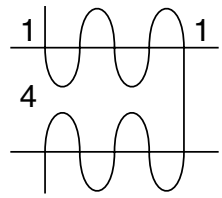

A15

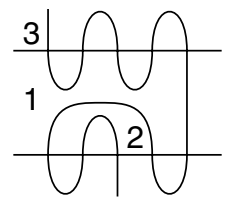

D3

3. 0123458769

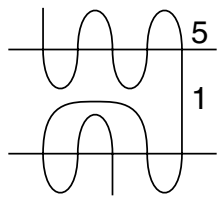

D14

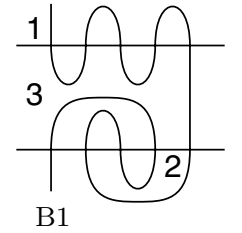

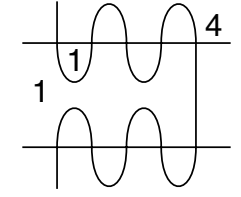

A12

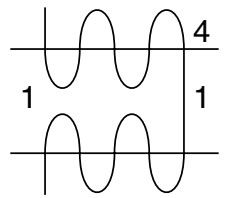

A13

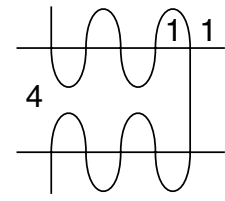

A14

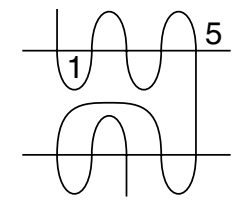

D7

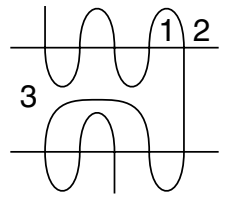

D11

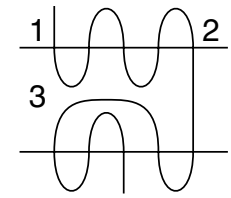

D13

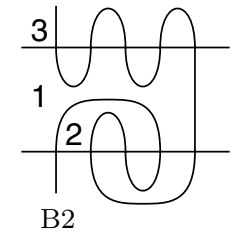

4. 0123476589
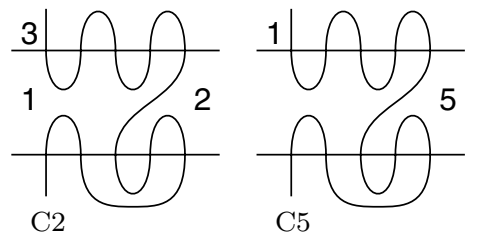
5. 0143256987

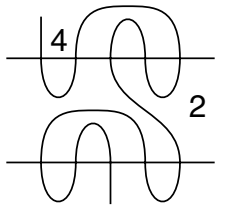

$\mathrm{F} 2$

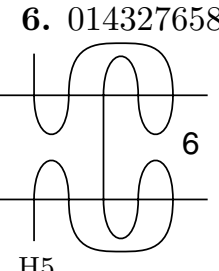

7. 0143278965

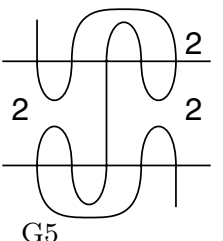

8. 0143296785

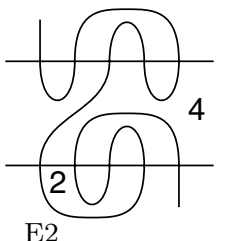

0.7. Pseudoholomorphic arrangements of $C_{5} \cup L_{1} \cup L_{2}$ of the type $(1,3)$. The list is organized as in Subsection 0.6, but in the encoding we assume that $d_{1}=1$ and $d_{2}=3\left(d_{1}\right.$ and $d_{2}$ are as in Subsection 0.4). Under some of the arrangements we refer to its construction (a singular curve to be perturbed). All others were constructed in [10].

1. 0127634589
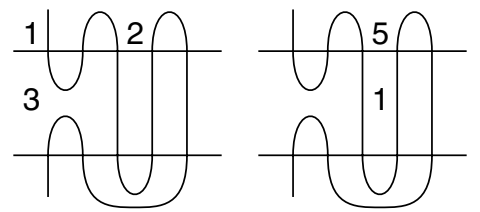

3. 0129678345
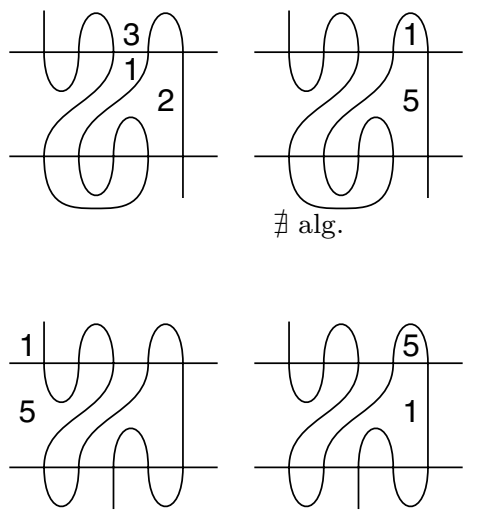

13.1
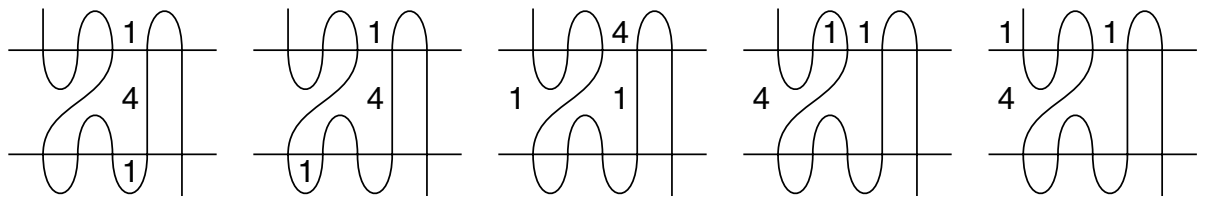

7. 0145632987
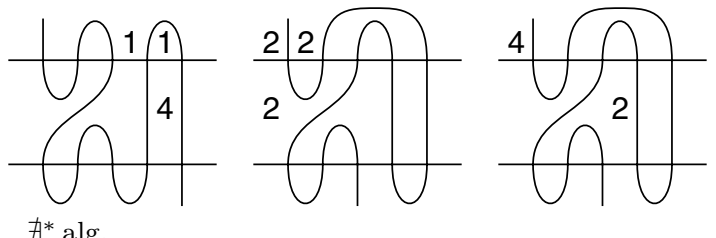

8. 0145678329
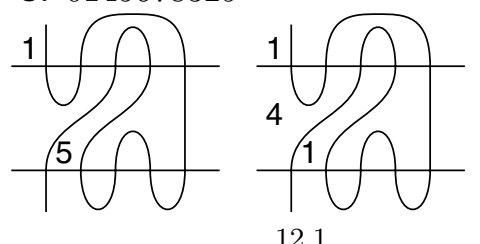


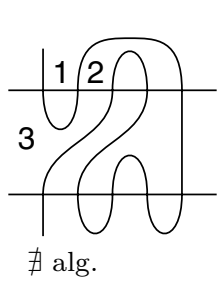

9. 0145876329

11. 0789612345

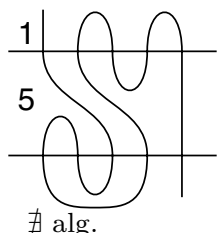

13. 0967812345

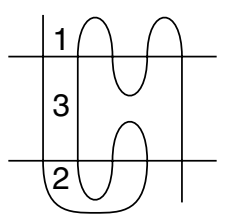

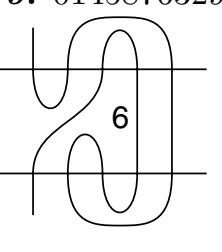

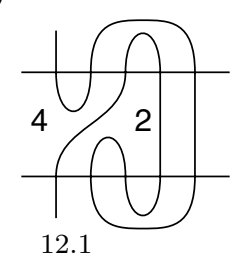

10. 0147832965

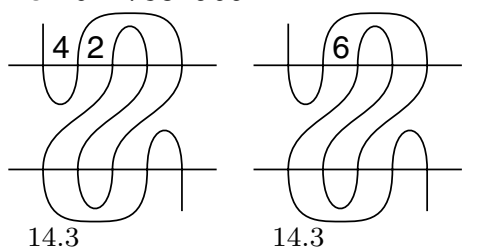

12. 0789632145
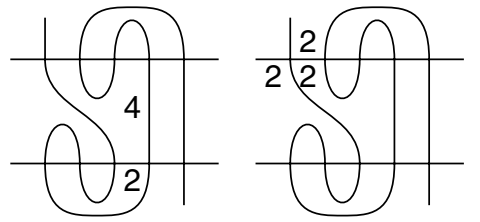

14. 0967832145

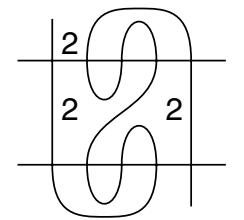

15. 0981234567
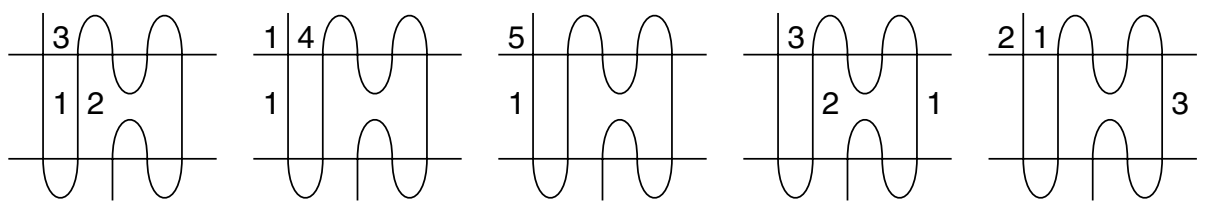
15.1-15.4

16. 0981432567

17. 0983214567
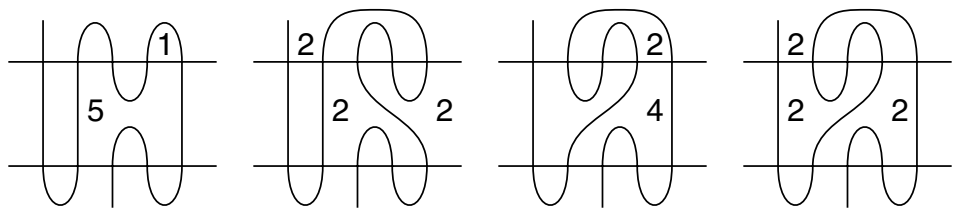

18. 0987612345

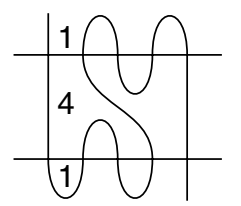

19. 2109834567
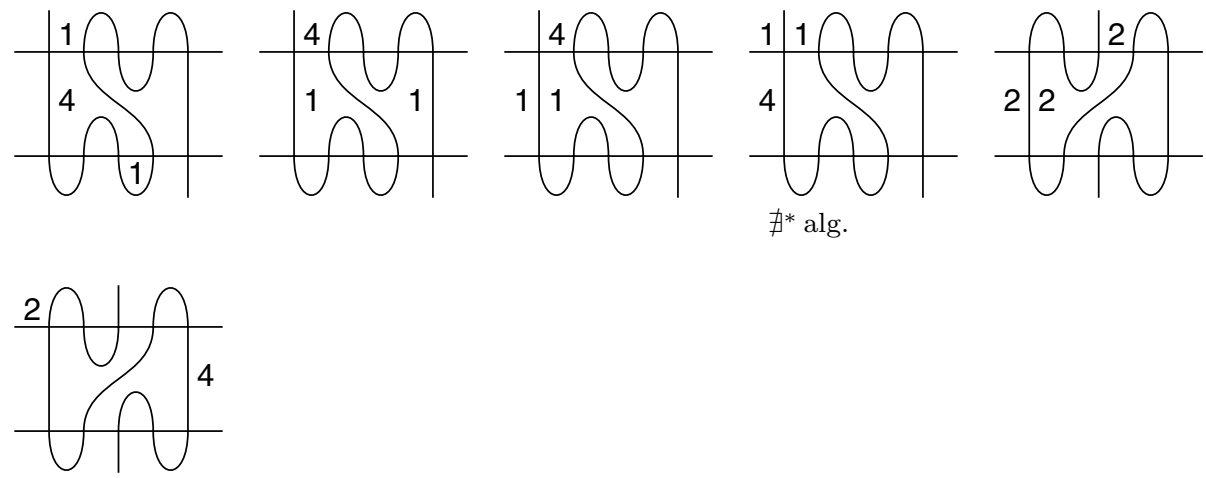


\section{§1. Curves on quadrics: The Formula of COMPleX ORIEnTATIONS, A Generalization of Fiedler's theOrem on symmetric $M$-CurVes, AND THE PROOF OF THEOREM 3}

1.1. Rohlin's formula of complex orientations and a characterization of hyperbolic curves on $\mathbb{R P}^{2}$. Let $A$ be a nonsingular real algebraic curve on $\mathbb{R P}^{2}$ (the set of its complex points), and let $\mathbb{R} A$ be the set of its real points. Suppose that the curve $A$ is dividing, i.e., $A \backslash \mathbb{R} A$ has two connected components; closures of them will be denoted by $A_{+}$and $A_{-}$. A complex orientation of $\mathbb{R} A$ is the boundary orientation coming from one of the halves $A_{+}$or $A_{-}$.

Let $\Pi_{+}$(respectively, $\Pi_{-}$) be the number of pairs of ovals $(O, o)$ of $\mathbb{R} A$ such that $o$ is inside $O$ and $[o]=-[O]$ (respectively, $[O]=[o]$ ) in the first homology group of the annulus bounded in $\mathbb{R P}^{2}$ by these ovals. Let $l$ be the number of ovals of $\mathbb{R} A$. Suppose that the degree of $A$ is even and is equal to $2 k$. Then the following Rohlin formula of complex orientations holds:

$$
2\left(\Pi_{+}-\Pi_{-}\right)=l-k^{2} .
$$

Now, suppose that the degree of $A$ is odd and is equal to $2 k+1$. Let $J$ be the odd branch of $A$. Let $\Lambda_{+}$(respectively, $\Lambda_{-}$) be the number of ovals $O$ such that $[O]=-2[J]$ (respectively, $[O]=2[J]$ ) in the first homology group of the exterior of $O$. Then the following Rohlin-Mishachev formula of complex orientations holds:

$$
\left(\Lambda_{+}-\Lambda_{-}\right)+2\left(\Pi_{+}-\Pi_{-}\right)=l-k^{2}-k .
$$

A consequence of (1) and (2) is the following fact, also discovered by Rohlin. Recall that a real algebraic curve on $\mathbb{R} \mathbb{P}^{2}$ is said to be hyperbolic if there exists a point $p \in \mathbb{R P}^{2}$ such that any real line through $p$ intersects $A$ at $d$ real points, where $d$ is the degree of $A$. This condition is equivalent to the statement that $\mathbb{R} A$ consists of $[d / 2]$ nested ovals, and also of the odd branch when $d$ is odd.

Proposition 1.1. Let $A$ be a dividing real algebraic curve on $\mathbb{R P}^{2}$ of degree $2 k$ or $2 k+1$ that has $l$ ovals. Then $l \geq k$, and if $l=k$, then $A$ is hyperbolic.

Proof. We give a proof for the case of even degree. When the degree is odd, the proof is similar. It is clear that $\left(l^{2}-l\right) / 2=$ (the number of pairs of ovals) $\geq$ (the number of nested pairs of ovals $)=\Pi_{-}+\Pi_{+} \geq \Pi_{-}-\Pi_{+}$, and the latter expression is equal to $\left(k^{2}-l\right) / 2$ by (1). This implies $l^{2} \geq k^{2}$, whence $l \geq k$. Moreover, if $l=k$, then we have equality in all the inequalities, so that each pair of ovals is nested.

1.2. Zvonilov's formula of complex orientations and a characterization of hyperbolic curves on an ellipsoid. Here we present Zvonilov's formula 24 of complex orientations for curves on an ellipsoid and rewrite it in a more invariant form, i.e., so that its ingredients do not depend on the choice of an auxiliary point. In the same way as for curves on $\mathbb{R P}^{2}$, from it we derive the characterization of the hyperbolic curves on an ellipsoid as the dividing curves that have the minimal possible number of ovals.

Let $X$ be a complex quadric given in $\mathbb{C P}^{3}$ by $x_{1}^{2}+x_{2}^{2}+x_{3}^{2}=x_{0}^{2}$, and let $\mathbb{R} X$ be the set of its real points, i.e., the ellipsoid given in $\mathbb{R P}^{3}$ by the same equation. Let $A$ be a nonsingular dividing real algebraic curve on $X$ of degree $2 k$ (in other words, of bidegree $(k, k))$. We fix a complex orientation on $\mathbb{R} A$.

We choose any point $p \in \mathbb{R} X \backslash \mathbb{R} A$. Let $O_{1}, \ldots, O_{l}$ be the ovals of $A$. Each $O_{i}$ splits $\mathbb{R} X$ into two disks. Let $D_{i}$ be the disk that does not contain $p$. We endow these disks with the orientation induced from $\mathbb{R} X$. We set $\varepsilon_{i}=1$ and say that an oval $O_{i}$ is positive if its complex orientation coincides with the boundary orientation induced from 
$D_{i}$. Otherwise we say that $O_{i}$ is negative and set $\varepsilon_{i}=-1$ (these definitions depend on the choice of $p$ ).

Let $\Lambda_{+}^{p}$ and $\Lambda_{-}^{p}$ be the number of positive and negative ovals, respectively, and let $\Pi_{ \pm}^{p}$ be the number of pairs $(i, j)$ such that $i<j, D_{i} \cap D_{j} \neq \varnothing$, and $\varepsilon_{i} \varepsilon_{j}=\mp 1$. Then the following formula of complex orientations holds (see 24] and also [2]):

$$
2 l-\left(\Lambda_{-}^{p}-\Lambda_{+}^{p}\right)^{2}+4\left(\Pi_{-}^{p}-\Pi_{+}^{p}\right)=k^{2} .
$$

Now, let us express the left-hand side of (3) in terms of quantities independent of the choice of $p$. Any pair of disjoint embedded circles splits a 2-sphere into three parts such that two of them are homeomorphic to a disk and the third is homeomorphic to an annulus. We say that a pair of ovals of $\mathbb{R} A$ is negative if they realize the same first homology class of the annulus bounded by them, and positive otherwise. Let $\Pi_{+}$and $\Pi_{-}$ be the number of positive and negative pairs of ovals of $\mathbb{R} A$.

Proposition 1.2. Formula (1) is true for any dividing curve of bidegree $(k, k)$ with $l$ ovals on an ellipsoid.

Proof. Let $\tilde{\Pi}_{ \pm}^{p}$ be the number of pairs $(i, j)$ such that $i<j, D_{i} \cap D_{j}=\varnothing$, and $\varepsilon_{i} \varepsilon_{j}= \pm 1$. Clearly, $\Lambda_{+}^{p}-\Lambda_{-}^{p}=\varepsilon_{1}+\cdots+\varepsilon_{l}$. Hence,

$$
\begin{aligned}
\left(\Lambda_{-}^{p}-\Lambda_{+}^{p}\right)^{2} & =\left(\sum_{i=1}^{l} \varepsilon_{i}^{2}\right)+2\left(\sum_{D_{i} \cap D_{j} \neq \varnothing} \varepsilon_{i} \varepsilon_{j}\right)+2\left(\sum_{D_{i} \cap D_{j}=\varnothing} \varepsilon_{i} \varepsilon_{j}\right) \\
& =l+2\left(\Pi_{-}^{p}-\Pi_{+}^{p}\right)-2\left(\tilde{\Pi}_{-}^{p}-\tilde{\Pi}_{+}^{p}\right) .
\end{aligned}
$$

Substituting $\tilde{\Pi}_{ \pm}^{p}=\Pi_{ \pm}-\Pi_{ \pm}^{p}$ and adding (3), we obtain (1).

We say that pairwise disjoint, smoothly embedded circles $O_{1}, \ldots, O_{r}$ form a nest if there exist disks $D_{1}, \ldots, D_{r}$ such that $\partial D_{i}=O_{i}$ and $D_{1} \subset D_{2} \subset \cdots \subset D_{r}$. In this case $r$ is called the depth of the nest. For example, two ovals always form a nest, but three ovals may fail to form a nest. A curve of bidegree $(k, k)$ on an ellipsoid is said to be hyperbolic if it contains a nest of depth $k$ (and hence, by Bézout's theorem, it cannot contain other ovals).

Proposition 1.3. Let $A$ be a dividing real algebraic curve on an ellipsoid, of bidegree $(k, k)$ and with $l$ ovals. Then $l \geq k$, and if $l=k$, then the curve $A$ is hyperbolic.

Proof. As in the proof of Proposition 1.1, formula (1) implies that $l \geq k$ and $\Pi_{+}=0$ for $l=k$. It remains to apply the following two evident facts:

1) if any two of three ovals form a negative pair, then all three ovals form a nest;

2 ) if any three of $k$ ovals form a nest, then all $k$ ovals also form a nest.

1.3. Fiedler's theorem on symmetric curves on $\mathbb{R P}^{2}$. Let $\left(x_{0}: x_{1}: x_{2}\right)$ be homogeneous coordinates on $\mathbb{R P}^{2} \subset \mathbb{C P}^{2}$. Let $s: \mathbb{C P}^{2} \rightarrow \mathbb{C P}^{2}$ be the holomorphic involution $\left(x_{0}: x_{1}: x_{2}\right) \mapsto\left(-x_{0}: x_{1}: x_{2}\right)=\left(x_{0}:-x_{1}:-x_{2}\right)$. The set $F$ of fixed points of this involution is the union of the line $F_{1}=\left\{x_{0}=0\right\}$ and the point $F_{0}=(1: 0: 0)$. Let $c: \mathbb{C P}^{2} \rightarrow \mathbb{C P}^{2}$ be the involution of the complex conjugation. We have $s \circ c=c \circ s$ (this involution is denoted by $\tilde{c}$ ). Any real (i.e., commuting with $c$ ) holomorphic involution of $\mathbb{C P}^{2}$ can be written in this form in suitable coordinates. Let $\widetilde{\mathbb{R P}}^{2}$ be the set of fixed points of $\tilde{c}$.

Let $A$ be a real algebraic curve on $\mathbb{R P}^{2}$ that is symmetric with respect to $s$. Set $\mathbb{R} A=A \cap \mathbb{R P}^{2}$ and $\widetilde{\mathbb{R} A}=A \cap \widetilde{\mathbb{R P}}^{2}$. We call $\widetilde{\mathbb{R} A}$ the complementary curve of $\mathbb{R} A$.

In coordinates, the property for the curve $A$ to be symmetric means that it is defined by an equation of the form $f\left(x_{0}^{2}, x_{1}, x_{2}\right)=0$. After the coordinate change $\tilde{x}_{0}=i x_{0}$, 
$\tilde{x}_{1}=x_{1}, \tilde{x}_{2}=x_{2}$, we see that $\widetilde{\mathbb{R P}}^{2}=\left\{\left(\tilde{x}_{0}: \tilde{x}_{1}: \tilde{x}_{2}\right) \mid \tilde{x}_{0}, \tilde{x}_{1}, \tilde{x}_{2} \in \mathbb{R}\right\}$ and $\widetilde{\mathbb{R} A}=\left\{\left(\tilde{x}_{0}\right.\right.$ : $\left.\left.\tilde{x}_{1}: \tilde{x}_{2}\right) \in \widetilde{\mathbb{R P}}^{2} \mid f\left(-\tilde{x}_{0}^{2}, \tilde{x}_{1}, \tilde{x}_{2}\right)=0\right\}$.

Theorem 1.4 (Fiedler [3]). Suppose that $\mathbb{R} A$ is an $M$-curve of degreed on $\mathbb{R P}^{2}$. Then:

a) if $\mathbb{R} A \cap F \neq \varnothing$, then $\widetilde{\mathbb{R} A}$ is a hyperbolic curve on $\widetilde{\mathbb{R P}}^{2}$;

b) if $d \notin\{2,4\}$, then $\mathbb{R} A \cap F \neq \varnothing$ (and hence, part (a) implies that $\widetilde{\mathbb{R} A}$ is a hyperbolic curve on $\widetilde{\mathbb{R P}}^{2}$ ).

Remark. A perturbation of the union of the conics $x_{0}^{2}=x_{1}^{2}+2 x_{2}^{2}$ and $x_{0}^{2}=2 x_{1}^{2}+x_{2}^{2}$ (respectively, any of them) provides an example showing that part (b) of Theorem 1.4 fails for $d=4$ (respectively, for $d=2$ ).

In the following subsection, we reproduce the proof of this theorem from [3], separating the intermediate statements into three types: those valid for abstract (i.e., nowhere embedded) symmetric real curves, those valid for curves on arbitrary surfaces, and those valid for curves on $\mathbb{R P}^{2}$. As a result, we shall obtain an analog of Theorem 1.4 for symmetric curves on a real quadric.

\subsection{Proof and generalization of Fiedler's theorem on symmetric curves.}

1.4.1. Abstract (nowhere embedded) curves. Let $A$ be a smooth Riemann surface of genus $g$ supplied with an antiholomorphic involution $c: A \rightarrow A$ and with a holomorphic involution $s: A \rightarrow A$ commuting with $c$. Set

$$
\tilde{c}=s \circ c=c \circ s, \quad \mathbb{R} A=\operatorname{Fix}(c), \quad \widetilde{\mathbb{R} A}=\operatorname{Fix}(\tilde{c}), \quad F=\operatorname{Fix}(s) .
$$

Lemma 1.5. If a curve $\mathbb{R} A$ is dividing and $F \cap \mathbb{R} A \neq \varnothing$, then $F=\mathbb{R} A \cap \widetilde{\mathbb{R} A}$.

Proof (see [3]). In a neighborhood of any point of $F \cap \mathbb{R} A$, the involution $s$ looks like $z \mapsto-z$. Hence, $s$ exchanges the halves of $A \backslash \mathbb{R} A$.

Lemma 1.6. Suppose that $\mathbb{R} A$ is an $M$-curve (i.e., the number of its connected components is $g+1)$. Then:

a) if $F \cap \mathbb{R} A \neq \varnothing$, then the curve $\widetilde{\mathbb{R} A}$ is dividing and the number of its connected components is one half the cardinality of $F$;

b) if $F \cap \mathbb{R} A=\varnothing$, then $\operatorname{Card} F$ is 0,2 , or 4 .

Proof (see [3]). We fill the holes of one of the halves of $A \backslash \mathbb{R} A$ by disks, extend $\tilde{c}$ to the resulting sphere, and apply Lemma 1.5.

Remark. Of course, Lemma 1.6 is an immediate consequence of the topological classification (obtained in [12]) of the pairs of commuting antiholomorphic involutions of Riemann surfaces.

1.4.2. Curves on arbitrary surfaces. Now, let $A$ be a nonsingular connected curve on a smooth surface $X$, and let $s, c, \tilde{c}:(X, A) \rightarrow(X, A)$ be a holomorhic and two antiholomorphic involutions such that $\tilde{c}=c \circ s=s \circ c$. Set $F=\operatorname{Fix}(s)=F_{0} \sqcup F_{1}$, where $\operatorname{dim} F_{0}=0, \operatorname{dim} F_{1}=1$, and denote

$$
\mathbb{R} Z=\operatorname{Fix}\left(\left.c\right|_{Z}\right), \quad \widetilde{\mathbb{R} Z}=\operatorname{Fix}\left(\left.\tilde{c}\right|_{Z}\right) \quad \text { for } Z=X, A, F, F_{k} .
$$

It is clear that $\mathbb{R} F_{k}=\widetilde{\mathbb{R} F}_{k}, k=0,1$.

Lemma 1.7. If $\mathbb{R} A$ is an $M$-curve and $\mathbb{R} A \cap F \neq \varnothing$, then $\widetilde{\mathbb{R} A}$ is a dividing curve whose number of components $b_{0}(\widetilde{\mathbb{R} A})$ satisfies the inequalities

$$
0 \leq 2 b_{0}(\widetilde{\mathbb{R} A})-\left(A \cdot F_{1}\right) \leq \operatorname{Card}\left(\mathbb{R} F_{0}\right) .
$$

Proof. This follows immediately from Lemma 1.6. 


\subsubsection{Curves on $\mathbb{R P}^{2}$ and on a real quadric.}

Proof of Theorem 1.4. By Lemma 1.6(b), we have $\mathbb{R} A \cap F \neq \varnothing$. Hence, by Lemma 1.7, the curve $\widetilde{\mathbb{R} A}$ is dividing and $2 b_{0}(\widetilde{\mathbb{R} A}) \leq \operatorname{deg} A+1$. It remains to apply Proposition 1.1 .

Now we proceed to the case where $\mathbb{R} X$ is a hyperboloid defined in $\mathbb{R} \mathbb{P}^{3}$ by the equation $x_{0}^{2}+x_{3}^{2}=x_{1}^{2}+x_{2}^{2}, X$ is its complexification, and $s: X \rightarrow X$ is the involution $\left(x_{0}: x_{1}:\right.$ $\left.x_{2}: x_{3}\right) \mapsto\left(x_{0}: x_{1}: x_{2}:-x_{3}\right)$. We introduce all the other notation as in Subsection 1.4.2 and set $\tilde{x}_{3}=i x_{3}$ and $\tilde{x}_{j}=x_{j}$ for $j \leq 2$. Then

$$
\widetilde{\mathbb{R} X}=\left\{\left(\tilde{x}_{0}: \cdots: \tilde{x}_{3}\right) \in X \mid \tilde{x}_{j} \in \mathbb{R}\right\}=\left\{\left(\tilde{x}_{0}: \cdots: \tilde{x}_{3}\right) \in \widetilde{\mathbb{R} P}^{3} \mid \tilde{x}_{0}^{2}=\tilde{x}_{1}^{2}+\tilde{x}_{2}^{2}+\tilde{x}_{3}^{2}\right\}
$$

is an ellipsoid. Clearly, $F_{0}=\varnothing$ and $F_{1}$ is a curve of bidegree $(1,1)$.

Theorem 1.8. Let $\mathbb{R} A$ be a nonsingular real $M$-curve on the hyperboloid $\mathbb{R} X$, of bidegree $(k, k)$ and symmetric with respect to $s$. Then:

a) if $\mathbb{R} A \cap F \neq \varnothing$, then the complementary curve $\widetilde{\mathbb{R} A}$ is a hyperbolic curve on the ellipsoid $\widetilde{\mathbb{R} X}$

b) if $k>2$, then $\mathbb{R} A \cap F \neq \varnothing$ (and hence, part (a) implies that $\widetilde{\mathbb{R} A}$ is a hyperbolic curve on $\widetilde{\mathbb{R} X}$ ).

Proof. Use Proposition 1.3 rather than Proposition 1.1 in the proof of Theorem 1.4.

1.5. Proof of Theorem 3. Let $\left(y_{0}: y_{1}: y_{2}\right)$ be coordinates on $\mathbb{R P}^{2}$ such that the conic $C_{2}$ is given by $y_{0}^{2}=y_{1}^{2}+y_{2}^{2}$. Let $X, c, s$ mean the same as in Subsection 1.4.3. Let $\xi: X \rightarrow \mathbb{C P}^{2}$ be the double covering $\left(x_{0}: x_{1}: x_{2}: x_{3}\right) \mapsto\left(x_{0}: x_{1}: x_{2}\right)$ branched along $C_{2}$. Then $\xi=\xi \circ s$; hence, $c$ and $\tilde{c}$ are two lifts to $X$ of the involution of the complex conjugation of $\mathbb{C P}^{2}$.

We denote the connected components of $\mathbb{R}^{2} \backslash \mathbb{R} C_{2}$ by $D$ (disk) and $M$ (Möbius band). It is easy to check that $\mathbb{R} X$ is a hyperboloid (topologically, a torus) and $\xi$ maps it onto $\bar{M}$ with a fold along $\mathbb{R} F_{1}$. Moreover, $\mathbb{R} F_{1}$ is mapped diffeomorphically onto $\mathbb{R} C_{2}$, and the restriction of $\xi$ to $\mathbb{R} X \backslash \mathbb{R} F_{1}$ (which is homeomorphic to an open annulus) is a connected double covering of $M$. Next, $\widetilde{\mathbb{R} X}$ is a 2-sphere, and $\xi$ maps it onto $\bar{D}$ with a fold along $\mathbb{R} F_{1}$. Moreover, $\widetilde{\mathbb{R} X} \backslash \mathbb{R} F_{1}$ is a disjoint union of two disks, each being mapped homeomorphically onto $D$.

Set $A=\xi^{-1}\left(C_{n}\right)$. This is a curve of bidegree $(n, n)$ on $X$. Hence, its genus is $g(A)=(n-1)^{2}$ (the number of integral points inside the square of size $\left.n \times n\right)$. Then $\mathbb{R} A$ is an $M$-curve on the hyperboloid $\mathbb{R} X$. Indeed, each oval of $C_{n}$ lying outside of $\mathbb{R} C_{2}$ (the number of them is $g\left(C_{n}\right)$ ) is zero homologous in $H_{1}(M)$ and, so, provides two ovals of $\mathbb{R} A$ on $\mathbb{R} X$, and each exterior arc of $B_{n}$ provides one oval on $\mathbb{R} X$. Thus, we have $2 g\left(C_{n}\right)+n=\left(n^{2}-3 n+2\right)+n=g(A)+1$ ovals, i.e., $\mathbb{R} A$ is an $M$-curve.

Therefore, Theorem 1.8 implies that $\widetilde{\mathbb{R} A}$ is a hyperbolic curve on the ellipsoid $\widetilde{\mathbb{R} X}$, and Theorem 3 follows.

\section{$\S 2$. Constructions}

2.1. Definitions and notation. Recall that a curve has a singularity of the type $A_{n}$ (respectively, of the type $E_{8}$ ) at a point $p$ if it can be defined by the equation $y^{2}= \pm x^{n+1}$ (respectively, by $y^{3}=x^{5}$ ) in suitable local analytic coordinates centered at $p$.

Definition 2.1. Suppose that a curve is nonsingular at a point $p$ and another curve has a singularity of the type $A_{n}$ at $p$. We say that these curves have a maximal (respectively, almost maximal) intersection at $p$ if the local intersection multiplicity is equal to $n+1$ (respectively, to $n$ ). 
Note that if a curve is nonsingular at a point $p$ and another curve has a singularity of the type $A_{2 k}$ at $p$, then the intersection is maximal if and only if one of the curves is situated on both sides of the other curve.

Notation 2.2. Let $C$ be a curve in $\mathbb{R P}^{2}$, and let $p$ be a nonsingular point of $C$ that is not a flex point. We choose coordinates $(x: y: z)$ so that the line $z=0$ is tangent to $C$ at $p$. Let a real parameter $a$ be such that the intersection multiplicity of the conic $y z=a x^{2}$ with $C$ is at least 3 at $p$. Let $f_{C, p}$ be the birational quadratic transformation $(x: y: z) \mapsto\left(x z: y z-a x^{2}: z^{2}\right)$, i.e., $(X, Y) \mapsto\left(X, Y-a X^{2}\right)$ in the affine coordinates $X=x / z, Y=y / z$.

Notation 2.3. Let $p$ and $q$ be two points in $\mathbb{R P}^{2}$, and let $L$ be a line passing through $q$ but not passing through $p$. We choose coordinates $(x: y: z)$ so that $p=(0: 1: 0)$, $q=(0: 0: 1)$, and $L=\{y=0\}$. Let $h_{p, q, L}$ be the birational quadratic transformation $(x: y: z) \mapsto\left(x^{2}: x y: y z\right)$. In the literature on the topology of real algebraic curves, this transformation is usually called a hyperbolism (Viro introduced this term, referring to Newton).

\subsection{Construction of some mutual arrangements of a singular quintic and a nonsingular conic.}

Lemma 2.4. There exist mutual arrangements as depicted in Figures 1.1, 1.2 of an $M$-quartic $C_{4}$ and three lines $L, L^{\prime}$, and $(p q)$.

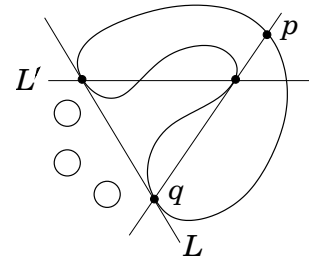

FIGURE 1.1

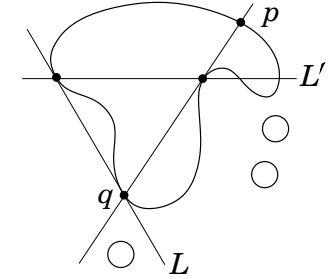

FiguRE 1.2

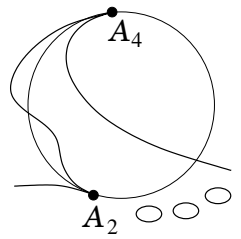

FiguRE 2.1

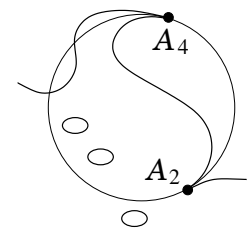

FIGURE 2.2

Proof. Figure 1.1 can easily be constructed starting with an $M$-quartic obtained as a perturbation of the union of two conics. A construction of the arrangement of Figure 1.2 is shown in Figures 3.1-3.3.

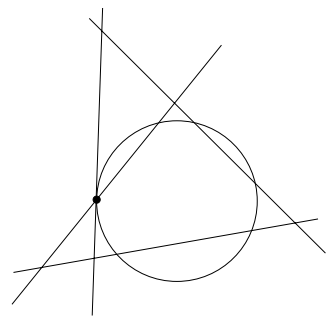

FiguRE 3.1

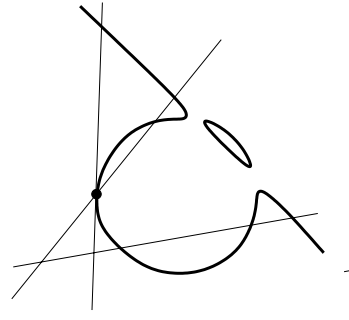

FiguRe 3.2

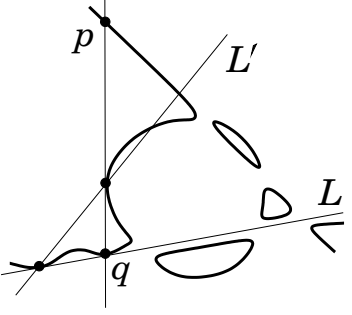

FIGURE 3.3

Lemma 2.5. There exist mutual arrangements as depicted in Figures 2.1, 2.2 of a singular four-component quintic $C_{5}$ with singularities $A_{2}$ and $A_{4}$ and a nonsingular conic $C_{2}$ (the curves have a maximal intersection at the singular points).

Proof. Apply $h_{p, q, L}$ to the curves in Lemma 2.4. Then $C_{4} \rightarrow C_{5}, L^{\prime} \rightarrow C_{2}$. 
Lemma 2.6. Let $C$ be a nonsingular $M$-quartic, and let $O$ be one of its ovals. Let $L_{1}$ be a line tangent to $O$ at points $p$ and $q$. Let $L_{2}$ be a line through $q$ that intersects $O$ at four distinct real points. Then $C, L_{1}$, and $L_{2}$ are arranged on $\mathbb{R P}^{2}$ as in one of Figures 4.1-4.3. All these arrangements are realizable.

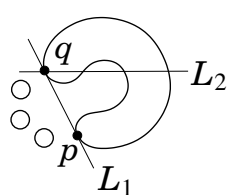

FiguRE 4.1

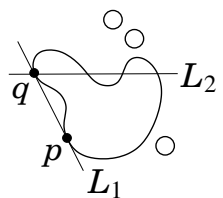

FiguRE 4.2

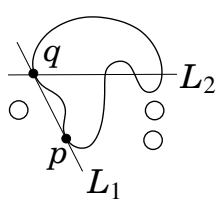

FigURE 4.3
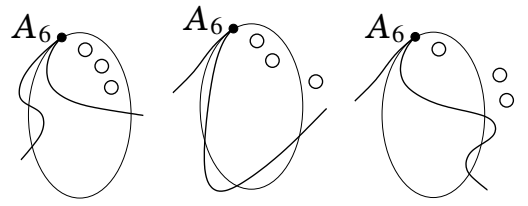

Figure 5.1 Figure 5.2 Figure 5.3

Proof. This follows easily from the classification of maximal mutual arrangements of an $M$-quartic and two lines. Moreover, Figure 4.1 and Figure 4.2 can be obtained from Figure 1.1 and Figure 1.2 (respectively) by forgetting one of the lines and changing the notation.

Lemma 2.7. Let $C_{2}$ be a nonsingular conic, and let $C_{5}$ be a singular four-component quintic whose odd branch $J_{5}$ has a singularity of type $A_{6}$. Suppose that $J_{5}$ and $C_{2}$ have a maximal intersection at $A_{6}$ and three transversal intersections. Then $C_{2}$ and $C_{5}$ are arranged on $\mathbb{R P}^{2}$ as in one of Figures 5.1-5.3. All these arrangements are realizable.

Proof. Apply $f_{C, p}$ to the arrangements in Lemma 2.6. Then $C \rightarrow C_{5}, L_{2} \rightarrow C_{2}$, and $q \mapsto A_{6}$.

Lemma 2.8. There exist mutual arrangements as depicted in Figures 6.1, 6.2 of a cuspidal cubic $C_{3}$, a nonsingular conic $C_{2}^{\prime}$, and two lines $L$ and $(p q)$.

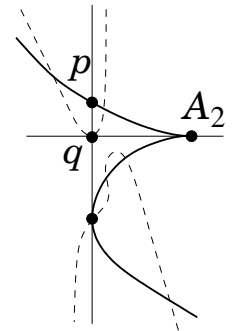

Figure 6.1

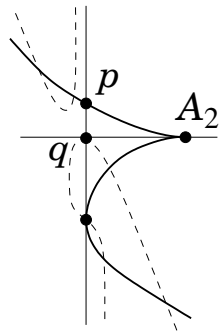

Figure 6.2

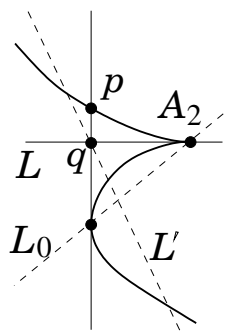

IGURE 6.3

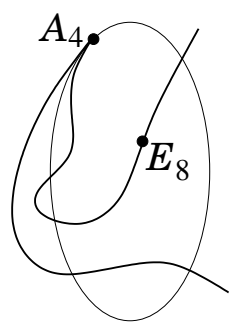

FigURE 7.1

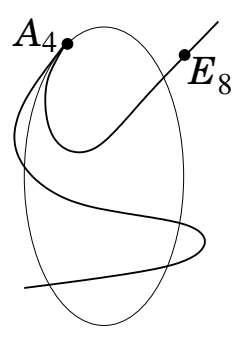

FiguRe 7.2

Proof. Clearly, if we forget the conic $C_{2}^{\prime}$, then the required arrangement exists (it is the same in both cases). Let $\ell, \ell^{\prime}, \ell^{\prime \prime}$, and $\ell_{0}$ be linear functions defining the lines $L, L^{\prime}$, $(p q)$, and $L_{0}$, respectively (see Figure 6.3). Set $C_{2}^{\prime}=\left\{\ell^{\prime} \ell^{\prime \prime}=\varepsilon \ell \ell_{0}\right\},|\varepsilon| \ll 1$. Then we obtain Figure 6.1 or Figure 6.2, depending on the sign of $\varepsilon$.

Lemma 2.9. There exist mutual arrangements as depicted in Figures 7.1, 7.2 of a rational quintic $C_{5}$ with singularities $A_{4}$ and $E_{8}$ and a nonsingular conic $C_{2}$ that have a maximal intersection at $A_{4}$.

Proof. Apply $h_{p, q, L}$ to the curves in Lemma 2.8. Then $C_{3} \rightarrow C_{5}, C_{2}^{\prime} \rightarrow C_{2}$. 
Definition 2.10. Let $\mathcal{F}_{n}$ denote a rational ruled surface (Hirzebruch surface) of degree $n$, i.e., a fiberwise compactification of the line bundle $\mathcal{O}(n)$ over $\mathbb{P}^{1}$. The surface $\mathcal{F}_{n}$ can be covered by affine coordinate charts $\left(x_{1}, y_{1}\right), \ldots,\left(x_{4}, y_{4}\right)$ with the transition functions

$$
\begin{array}{cccc}
x_{2}=x_{1}^{-1}, & y_{2}=y_{1} x_{1}^{-n}, & x_{4}=x_{3}^{-1}, & y_{4}=y_{3} x_{3}^{n}, \\
x_{3}=x_{1}, & y_{3}=y_{1}^{-1}, & x_{4}=x_{2}, & y_{4}=y_{2}^{-1}
\end{array}
$$

(these are the toric coordinates corresponding to the fan spanned by the vectors $(1,0)$, $(0, \pm 1),(-1, n))$. In this case, the coordinates $\left(x_{1}, y_{1}\right)$ are said to be standard. We shall only consider the real structure on $\mathcal{F}_{n}$ for which the standard coordinates are real.

In standard coordinates, the fibration $\mathcal{F}_{n} \rightarrow \mathbb{P}^{1}$ is the projection onto the $x_{1}$-axis. The exceptional section is defined by $y_{3}=0$ or $y_{4}=0$. We shall depict $\mathbb{R} \mathcal{F}_{n}$ as a rectangle whose opposite sides are identified. The horizontal sides correspond to the exceptional section; the vertical sides correspond to some fiber.

The bidegree of a curve $C$ on $\mathcal{F}_{n}$ is the pair $(k, m)$, where $k$ and $m$ are the intersection numbers of $C$ with $x_{1}=0$ and with $y_{1}=0$, respectively.

Lemma 2.11. Let $C, G$, and $F$ be real curves of bidegrees $(2,8),(1,4)$, and $(0,1)$, respectively, on $\mathcal{F}_{4}\left(G\right.$ is a section and $F$ is a fiber of $\left.\mathcal{F}_{4} \rightarrow \mathbb{P}^{1}\right)$. Suppose that $C$ is an $M$-curve (i.e., it has four ovals) that intersects $G$ at eight real points. Suppose that all the intersection points lie on the same oval of $C$ and that $F$ is tangent to $C$ at one of them. 8.1

Then $C, G$, and $F$ are arranged on $\mathbb{R} \mathcal{F}_{4}$ as in one of Figures 8.1-8.4. All these arrangements are realizable.

Moreover, any free (i.e., nonintersecting with $G$ ) oval can be replaced by a solitary simple double point.

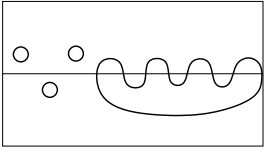

Figure 8.1

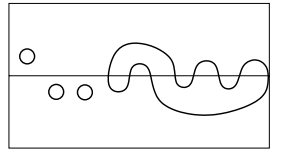

FIGURE 8.2

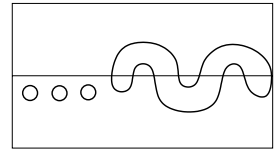

FIGURE 8.3

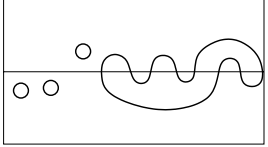

FiguRe 8.4

Proof. The curves in Figures 8.1-8.4 can be produced, for example, by Viro's T-construction (patchwork) as was done in [20, §2.2]. They can also be produced by applying a general method of construction of trigonal curves on ruled surfaces [17.

The nonexistence of other arrangements easily follows, for example, from the general algorithm of realizability recognition for real pseudoholomorphic trigonal curves [19].

The fact that any oval of a trigonal curve can be replaced by a solitary double point without changing the fiberwise arrangement of the rest of the curve, was proved in 17 , Lemma 2].

Lemma 2.12. Let $C_{2}$ be a nonsingular conic, and let $C_{5}$ be a three-component quintic whose odd branch $J_{5}$ has a singularity of type $E_{8}$. Suppose that $C_{2}$ passes through $E_{8}$ and also intersects $J_{5}$ at seven other points. Then $C_{2}$ and $C_{5}$ are arranged on $\mathbb{R P}^{2}$ as in one of Figures 9.1-9.8 (we do not depict the ovals of $C_{5}$ lying in the component of $\mathbb{R P}^{2} \backslash\left(C_{2} \cup J_{5}\right)$ whose closure is nonorientable). All these arrangements are realizable.

Remark. The dashed line in Figures $9.1-9.8$ is the tangent line to $C_{5}$ at $E_{8}$. The local arrangement of $C_{5}$ at $E_{8}$ with respect to this line is important for perturbations. 


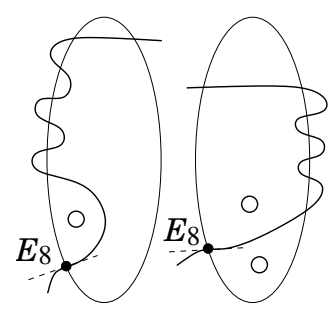

Figure 9.1

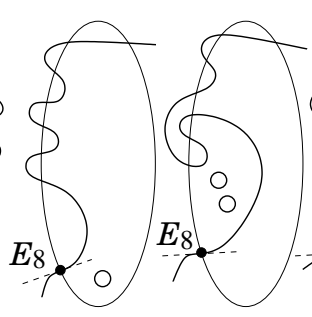

Figure 9.3

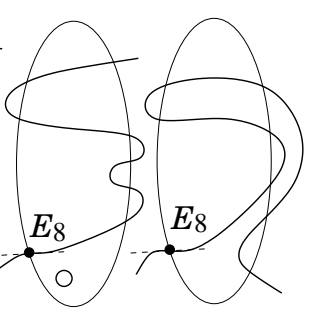

Figure 9.5

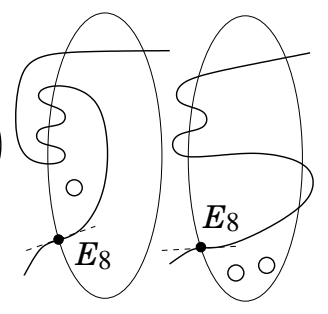

FiguRE 9.7

Figure 9.2

FiguRe 9.4

Figure 9.6

FiguRE 9.8

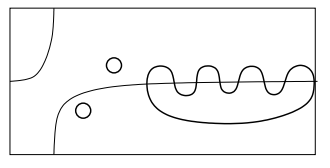

FIGURE 10.1

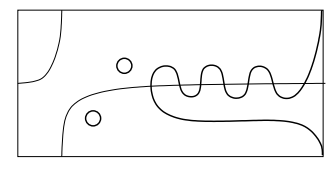

Figure 10.2

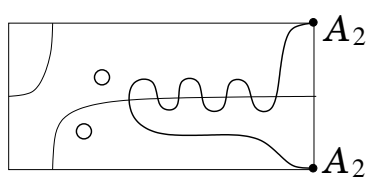

Figure 10.3

Proof. Let us choose one of the arrangements in Lemma 2.11 and contract one of its ovals into a double point. We blow up this point, and after that, blow down the proper transform of the fiber passing through it. This transforms $C, G$, and $F$ into curves $C^{\prime}, G^{\prime}$, and $F^{\prime}$ on $\mathcal{F}_{3}$ of bidegrees $(2,7),(1,4)$, and $(0,1)$, respectively. Their arrangement on $\mathcal{F}_{3}$ is depicted in Figure 10.1 for the choice of the leftmost oval in Figure 8.1. Now we perform such a transformation twice at the intersection point of $F^{\prime}$ and $G^{\prime}$. Then the curves $C^{\prime}$ and $G^{\prime}$ are transformed into curves $C^{\prime \prime}$ and $G^{\prime \prime}$ on $\mathcal{F}_{1}$ of bidegrees $(2,5)$ and $(1,2)$, respectively, arranged as in Figure 10.3 (the result of the intermediate transformation is depicted in Figure 10.2). The curve $C^{\prime \prime}$ has a cusp (a singularity $A_{2}$ ) where $C^{\prime \prime}$ has a maximal intersection with the exceptional section. Finally, we blow down the exceptional section, obtaining the required arrangement on $\mathbb{R P}^{2}$. Applying this construction to every arrangement in Lemma 2.11 and to every choice of the free oval, we arrive at Figures 9.1-9.8. The correspondence between pictures is the following (here $n_{k}$ means the choice of the $k$ th oval from the left in Figure 8.n):

$$
\begin{array}{cccc}
1_{1} \rightarrow 9.1, & 1_{2} \rightarrow 9.2, & 1_{3} \rightarrow 9.3, & 2_{1} \rightarrow 9.4 \\
\left(2_{2}, 2_{3}\right) \rightarrow 9.5, & \left(3_{1}, 3_{2}, 3_{3}\right) \rightarrow 9.6, & \left(4_{1}, 4_{2}\right) \rightarrow 9.7, & 4_{3} \rightarrow 9.8 .
\end{array}
$$

The proof is complete.

Lemma 2.13. There exists a mutual arrangement as depicted in Figure 11.4 of a singular six-component quintic $C_{5}$ with a singularity $A_{2}$ and a nonsingular conic $C_{2}$ that have a maximal intersection at the singular point.

Proof. See Figures 11.1-11.4.

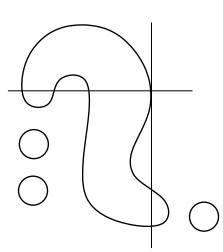

Figure 11.1

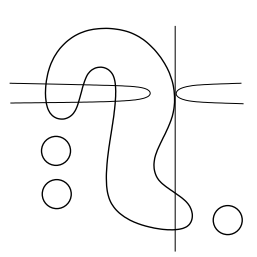

FiguRE 11.2 FiguRE 11.3

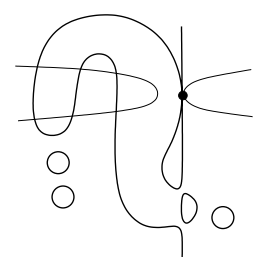

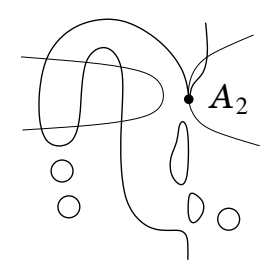

FiguRE 11.4 


\subsection{Construction of some mutual arrangements of a singular quintic and two} lines.

Lemma 2.14. There exists a quintic with singularities $A_{1}$ and $A_{3}$ arranged with respect to lines $L_{1}, L_{2}$ as in Figure 12.1.

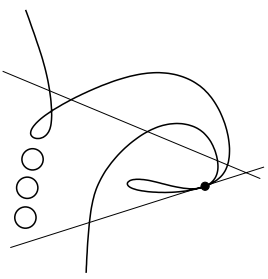

FIGURE 12.1

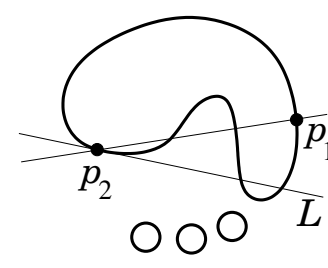

FiguRE 12.2
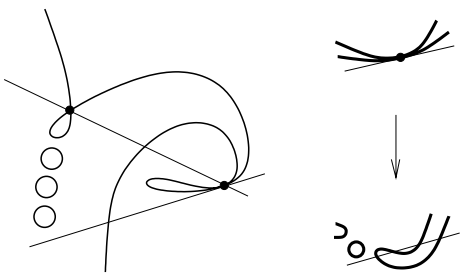

Figure 12.3 Figure 12.4

Proof. Consider a quartic arranged with respect to two lines as in Figure 12.2 and apply the hyperbolism $h_{p_{1}, p_{2}, L}$ to it. As a result, we obtain the arrangement in Figure 12.3. By a small shift of the upper line, it can be transformed into Figure 12.1.

The second arrangement of the series $\mathbf{9}$ on the list in Subsection 0.7 is constructed by applying the perturbation shown in Figure 12.4 to Figure 12.1.

Lemma 2.15. There exists a quintic with singularities $A_{2}$ and $E_{8}$ arranged with respect to lines $L_{1}, L_{2}$ as in Figure 13.1.

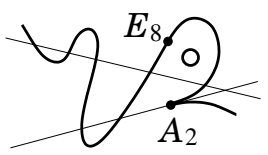

FiguRe 13.1

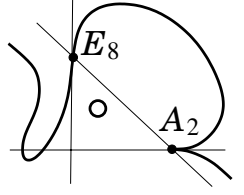

FiguRE 13.2

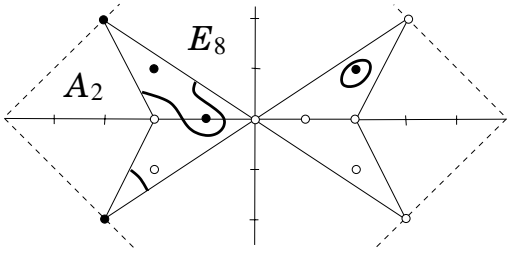

FigURE 13.3

Proof. It is easy to construct a curve as in Figure 13.2 using the Viro patchworking (see the corresponding subdivision into charts in Figure 13.3). This means simply that the curve in Figure 13.2 is given in homogeneous coordinates $(x: y: z)$ by the equation

$$
z^{5}+a x z^{4}+x^{2} z^{3}+x^{3} y^{2}-b x^{2} y z^{2}=0 \quad \text { for } 1 \ll a \ll b .
$$

Let the axis $y=0$ be chosen as the line $L_{1}$, and let $L_{2}$ be the line obtained from it by a small rotation clockwise around $A_{2}$ (this is the point (1:0:0)) followed by a yet smaller shift upward.

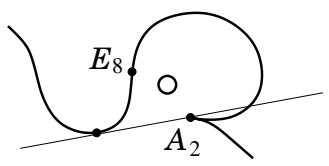

Figure 14.1

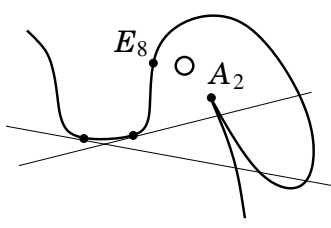

FiguRe 14.2

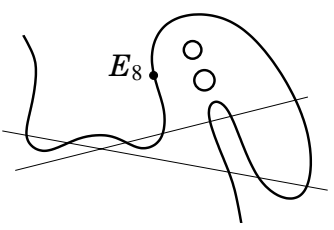

Figure 14.3 
Figure 14.1 can be obtained from Figure 13.2 by the rotation of the axis $y=0$ clockwise around $A_{2}$ until the first tangency with the quintic. Figure 14.2 can be obtained from Figure 14.1 by replacing the depicted tangent line with two close tangents. Figure 14.3 is a perturbation of Figure 14.2.

Construction of the second arrangement of the series 15 . Consider an $M$-quartic obtained by a small perturbation of the union of two conics. Let $V$ be one of its ovals, and let $q$ be a flex point on $V$. Let $\ell$ be a line through $q$ intersecting $V$ at four points. Let $p_{1}$ be the intersection point for which the segment $\left[q p_{1}\right]$ lies inside $V$. We choose a point $p_{0}$ on the concave part of $V$, consider the tangent to $V$ at $p_{0}$, and denote its intersection points with $V$ by $p_{2}$ and $p_{3}$ so that $p_{2}$ is closer to $q$ than $p_{3}$ in the sense that $\ell$ meets the arc $q p_{2}$ of $V$ only at $q$. Clearly, each of the lines $p_{1} p_{0}$ and $p_{1} p_{2}$ tends to $\ell$ as $p_{0} \rightarrow q$. Therefore, if the point $p_{0}$ is chosen sufficiently close to $q$, we obtain an arrangement of an $M$-quartic with respect to four lines as in Figure 15.1.

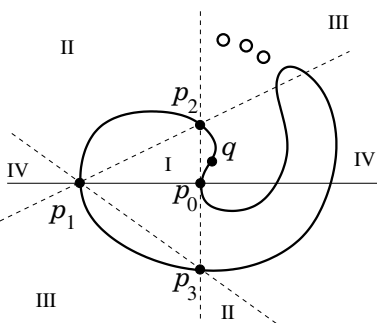

FIGURE 15.1

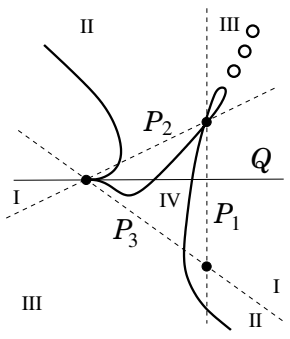

FIGURE 15.2
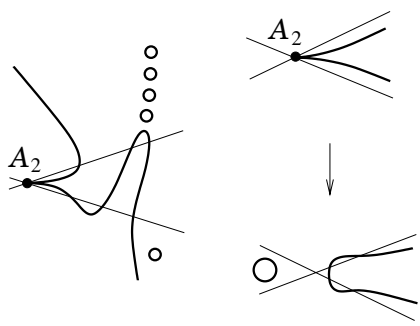

Figure 15.3 Figure 15.4

Applying the birational quadratic transformation centered at $p_{1}, p_{2}, p_{3}$, we obtain a singular quintic with singularities $A_{1}, A_{1}$, and $A_{2}$, arranged with respect to the lines $P_{1}$, $P_{2}, P_{3}, Q$ (that are the transforms of the points $p_{1}, p_{2}, p_{3}$ and the line $p_{1} p_{0}$, respectively), as in Figure 15.2, where the Roman numerals I, ..., IV indicate the correspondence among quarters.

Let $L_{1}$ and $L_{2}$ be two lines through $A_{2}$ that are close to $Q$ and placed on different sides of it. Perturbing simple double points, we obtain Figure 15.3. Finally, we perturb $A_{2}$ as shown in Figure 15.4.

\section{§3. Classification of pseudoholomorphic arrangements of a QuintiC AND TWO LINES OF THE TYPE $(3,3)$}

Proposition 3.1. a) Let $C_{5}$ be a real pseudoholomorphic (for example, algebraic) $M$ quintic in $\mathbb{R P}^{2}$, and let $L_{1}, L_{2}$ be two lines each of which intersects the odd branch $J_{5}$ of $C_{5}$ at five distinct points. Let $\mathcal{L}$ be the pencil of lines that contains $L_{1}$ and $L_{2}$. Suppose that the mutual arrangement of the curves $C_{5}, L_{1}, L_{2}$ is of the type $(3,3)$. Then $C_{5}, L_{1}$, and $L_{2}$ are arranged as in one of Figures 16.1-16.22 up to isotopy. Moreover, the fiberwise arrangement of $C_{5}$ with respect to $\mathcal{L}$ is as indicated in the caption to the corresponding figure up to insertion of zigzags of the form $\subset_{j} \supset_{j \pm 1}$ (see Remark 3.2).

b) All the arrangements in Figures 16.1-16.22 are realizable by real pseudoholomorphic curves. All of them except that in Figure 16.12 are realizable by real algebraic curves.

c) The arrangement in Figure 16.12 is algebraically unrealizable.

Remark 3.2. In Figures 16.1-16.22, we present each arrangement in three forms. In two of them, $\mathbb{R P}^{2}$ is cut along one of the lines. The third form is somewhat similar to the singular curve whose perturbation provides the given arrangement. 

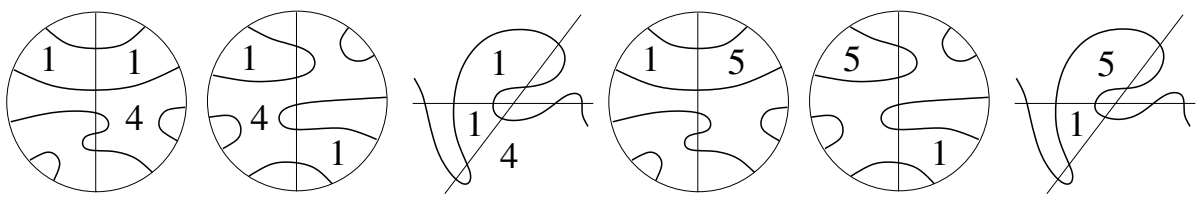

FiguRE 16.1. [ $\supset_{1} \mathrm{O}_{3} \subset_{1} \supset_{2} \mathrm{O}_{3} \mathrm{O}_{2} \mathrm{O}_{2} \mathrm{O}_{2} \mathrm{O}_{2} \subset_{2}$ ]. FigURE 16.2. [ $\supset_{1} \mathrm{O}_{3} \subset_{1} \supset_{2} \mathrm{O}_{3} \mathrm{O}_{3} \mathrm{O}_{3} \mathrm{O}_{3} \mathrm{O}_{3} \subset 2$ ].
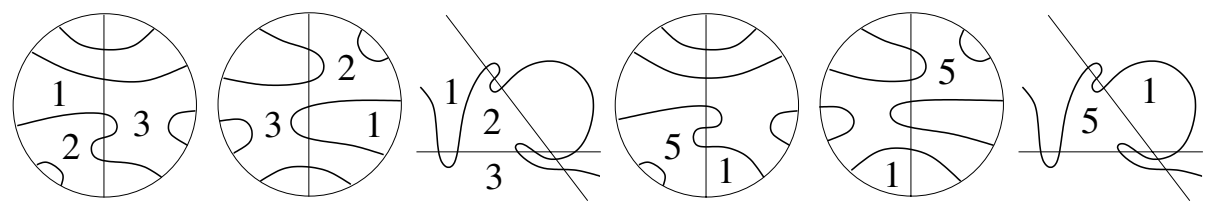

Figure 16.3. [ $\supset_{1} o_{1} O_{1} O_{2} \subset_{1} \supset_{2} O_{2} O_{2} O_{2} \subset \subset_{2}$ ]. FiguRE 16.4. [ $\supset_{1} o_{1} o_{1} o_{1} o_{1} o_{1} \subset_{1} \supset_{2} O_{1} \subset_{2}$ ].
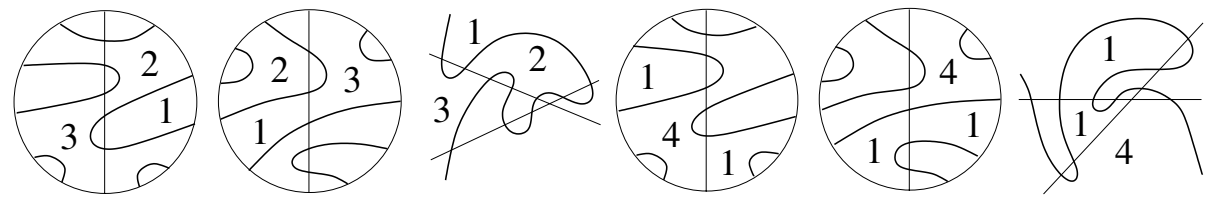

Figure 16.5. [ $\supset_{1} O_{1} O_{1} O_{1} \subset_{1} \supset_{3} O_{3} O_{3} O_{2} \subset_{1}$ ]. Figure 16.6. [ $\left.\supset_{1} O_{2} O_{1} O_{1} o_{1} o_{1} \subset_{1} \supset_{3} O_{1} \subset_{1}\right]$.
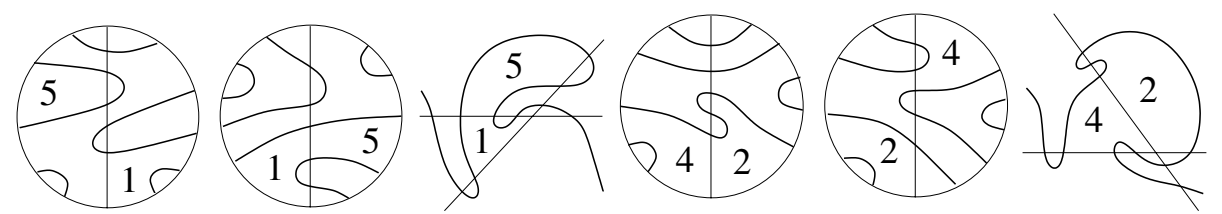

Figure 16.7. [ $\supset_{1} O_{2} O_{2} O_{2} O_{2} O_{2} \subset_{1} \supset_{3} O_{1} \subset_{1}$ ]. Figure 16.8. [ $\supset_{1} O_{1} O_{1} O_{1} O_{1} \subset_{2} \supset_{1} O_{1} O_{1} \subset_{2}$ ].
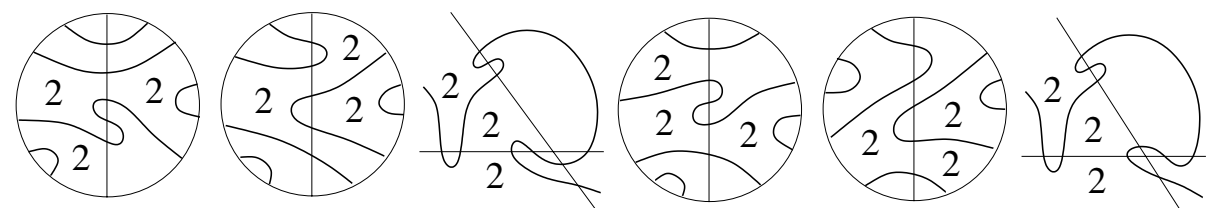

Figure 16.9. [ $\supset_{1} O_{1} O_{1} O_{2} O_{2} \subset_{2} \supset_{1} O_{2} O_{2} \subset_{2}$ ]. Figure 16.10. [ $\supset_{1} O_{3} O_{3} O_{2} O_{2} \subset_{2} \supset_{3} O_{2} O_{2} \subset_{2}$ ].
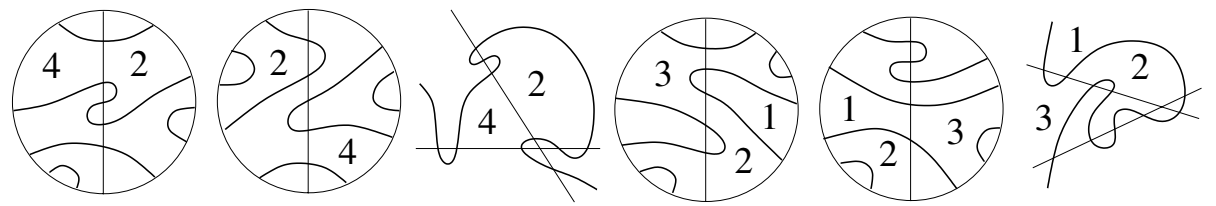

(Algebraically unrealizable)

FiguRE 16.11. [ $\supset_{1} \mathrm{O}_{3} \mathrm{O}_{3} \mathrm{O}_{3} \mathrm{O}_{3} \subset_{2} \supset_{3} \mathrm{O}_{3} \mathrm{O}_{3} \subset_{2}$ ]. FigURE 16.12. [ $\left.\supset_{1} \mathrm{O}_{3} \mathrm{O}_{3} \mathrm{O}_{3} \subset \subset_{3} \supset_{1} \mathrm{O}_{1} \mathrm{O}_{1} \mathrm{O}_{2} \subset \subset_{3}\right]$.
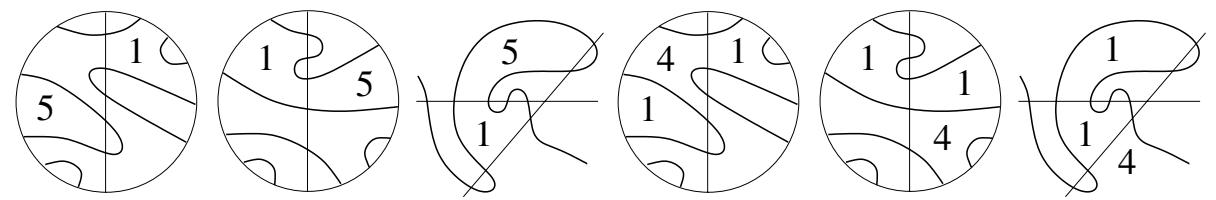

FiguRE 16.13. [ $\supset_{1} O_{2} O_{2} O_{2} O_{2} O_{2} \subset_{3} \supset_{1} O_{3} \subset_{3}$ ]. Figure 16.14. [ $\supset_{1} O_{2} O_{3} O_{3} O_{3} O_{3} \subset_{3} \supset_{1} O_{3} \subset \subset_{3}$ ]. 

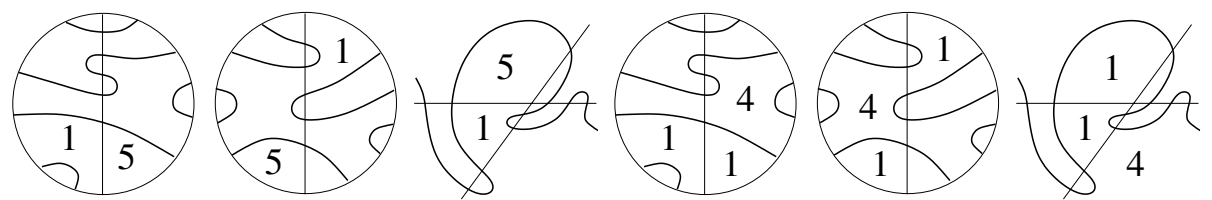

Figure 16.15. [ $\supset_{1} O_{1} \subset_{3} \supset_{2} O_{1} O_{1} O_{1} o_{1} O_{1} \subset_{2}$ ]. FiguRE 16.16. [ $\supset_{1} O_{1} \subset_{3} \supset_{2} O_{1} O_{2} O_{2} O_{2} O_{2} \subset_{2}$ ].
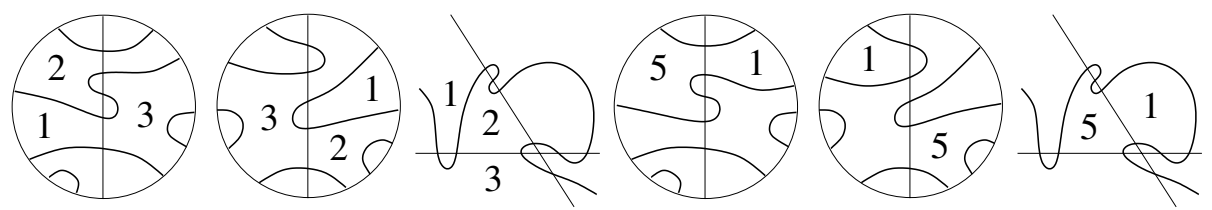

FiguRE 16.17. [ $\supset_{1} \mathrm{O}_{3} \mathrm{O}_{3} \mathrm{O}_{2} \subset_{3} \supset_{2} \mathrm{O}_{2} \mathrm{O}_{2} \mathrm{O}_{2} \subset_{2}$ ]. Figure 16.18. [ $\supset_{1} \mathrm{O}_{3} \mathrm{O}_{3} \mathrm{O}_{3} \mathrm{O}_{3} \mathrm{O}_{3} \subset_{3} \supset_{2} \mathrm{O}_{3} \subset_{2}$ ].
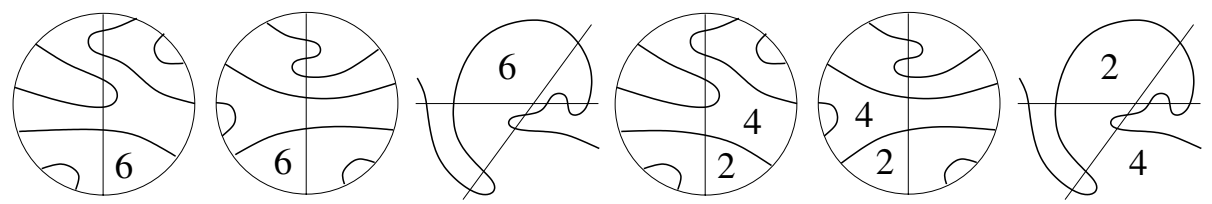

Figure 16.19. [ $\left.\supset_{1} \subset_{4} \supset_{2} O_{1} O_{1} O_{1} O_{1} O_{1} O_{1} \subset \subset_{3}\right]$. Figure 16.20. [ $\left.\supset_{1} \subset_{4} \supset_{2} O_{2} O_{2} O_{2} O_{2} O_{1} O_{1} \subset_{3}\right]$.
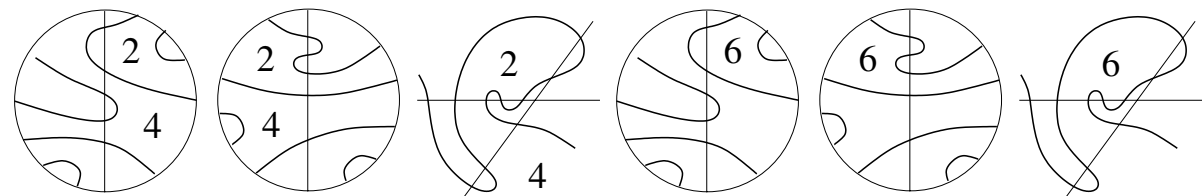

FiguRE 16.21. [ $\left.\supset_{1} \subset_{4} \supset_{2} O_{2} O_{2} O_{2} O_{2} O_{3} O_{3} \subset_{3}\right]$. Figure 16.22. [ $\left.\supset_{1} \subset_{4} \supset_{2} O_{3} O_{3} O_{3} O_{3} O_{3} O_{3} \subset_{3}\right]$.

In the captions, we give the fiberwise arrangement of $C_{5}$ with respect to $\mathcal{L}$ encoded as described in [16, §2] (see also Subsection 6.2 below), assuming that one of the lines $L_{1}$, $L_{2}$ is chosen as the infinite line. All the encoding words have the form

$$
\left[\supset_{a} o_{i_{1}} \ldots o_{i_{k}} \subset_{b} \supset_{c} o_{i_{k+1}} \ldots o_{j_{6}} \subset_{d}\right] .
$$

Depending on the choice of the infinite line and the orientations on it and on the pencil, eight encoding words are possible. We choose always the word for which the vector $[a, b, c, d]$ is the minimal possible with respect to the lexicographic order. Figures 16.116.22 are numbered in the ascending order of the vectors $\left[a, b, c, d, k, i_{1}, \ldots, i_{6}\right]$.

Restrictions. Proof of part a) of Proposition 3.1. The vector $[a, b, c, d]$ discussed in Remark 3.2 encodes the mutual arrangement of $J_{5}, L_{1}$, and $L_{2}$. It is clear that it must satisfy the conditions

$$
1 \leq a, b, c, d \leq 4, \quad b \neq c, \quad d \neq 5-a, \quad a+b+c+d \equiv 0 \quad \bmod 2 .
$$

The dihedral group of order 8 acts on the set of such vectors. It is generated by the mappings

$$
[a, b, c, d] \mapsto[c, d, 5-a, 5-b] \quad \text { and } \quad[a, b, c, d] \mapsto[d, c, b, a] .
$$

Vectors in the same orbit determine the same mutual arrangement of $J_{5}, L_{1}$, and $L_{2}$ up to swapping the roles of the lines and changing the orientations.

Taking the restrictions (5) and the symmetries (6) into account, we have 8 vectors to consider:

$$
[1,1,2,2],[1,1,3,1],[1,2,1,2],[1,2,3,2],[1,3,1,3],[1,3,2,2],[1,4,2,3],
$$




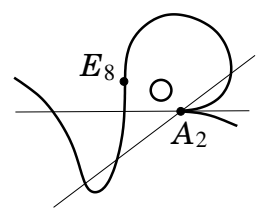

FIGURE 17

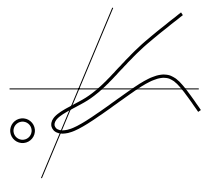

FiguRe 18.1

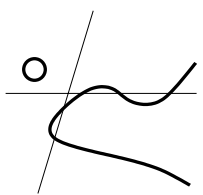

FiguRE 18.5

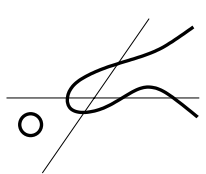

FiguRE 18.2

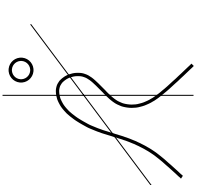

FiguRE 18.6

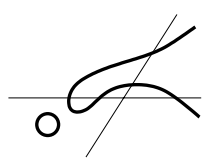

FIGURE 18.3

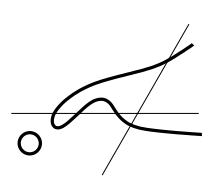

FIGURE 18.4

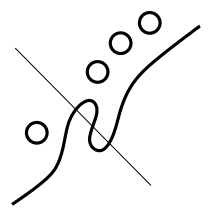

Figure 19.1

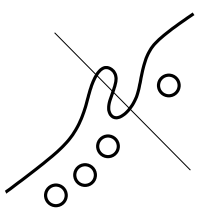

FiguRE 19.2

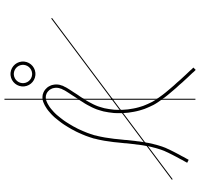

Figure 18.7

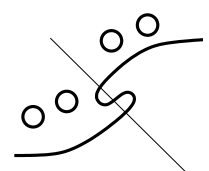

Figure 19.3

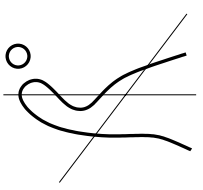

Figure 18.8

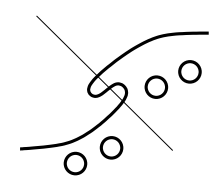

Figure 19.4

and $[1,2,4,1]$. However, the last vector does not provide any connected curve $J_{5}$.

The formula of complex orientations (2) implies that an $M$-quintic has three positive ovals and three negative ones. It is easy to deduce that the word (4) must satisfy the condition

$$
k \equiv a+b \quad \bmod 2 .
$$

Finally, it follows from Bézout's theorem for auxiliary lines that

$$
\begin{array}{cc}
\left|i_{j}-i_{l}\right| \leq 1 & \text { for } 1 \leq j<l \leq k \text { or } k<j<l \leq 6, \\
\left|i_{j}-a\right| \leq 2,\left|i_{j}-b\right| \leq 2 & \text { for } 1 \leq j \leq k, \\
\left|i_{j}-c\right| \leq 2,\left|i_{j}-d\right| \leq 2 & \text { for } k<j \leq 6 .
\end{array}
$$

For each word of the form (4), where the vector $[a, b, c, d]$ is one of $(7)$, the integers $k ; i_{1}, \ldots, i_{6}$ satisfy $(8)$ and $(9)$, and $1 \leq i_{j} \leq 4$, we have performed the following computations.

(1) Find the braid. All the links that are the closures of the resulting braids have three components.

(2) Check if all pairwise linking numbers of the link components are zero.

(3) If yes, then using the program in [16; Appendix], check if the Murasugi-Tristram inequality is fulfilled.

As a result, we have obtained only the words of the form (4) that occur in the captions to Figures 16.1-16.22.

Constructions. Proof of Part b) of Proposition 3.1. By a small rotation of the horizontal axis around the point $A_{2}$ in Figure 13.2, we obtain Figure 17. Perturbing the singular point $A_{2}$ as shown in Figures $18.1-18.5$ and Figure 18.8 and perturbing 
each time $E_{8}$ in two possible ways, we obtain the arrangements in Figure 16. $n$ for $n=$ $1,2,6,7,13,14,15,16,19,20,21,22$ as follows:
$18.1 \rightarrow\left\{\begin{array}{l}16.15 \\ 16.16\end{array}\right.$
$18.2 \rightarrow\left\{\begin{array}{l}16.1 \\ 16.2\end{array}\right.$
$18.3 \rightarrow\left\{\begin{array}{l}16.6 \\ 16.7\end{array}\right.$
$18.4 \rightarrow\left\{\begin{array}{l}16.13 \\ 16.14\end{array}\right.$
$18.5 \rightarrow\left\{\begin{array}{l}16.19 \\ 16.20\end{array}\right.$
$18.8 \rightarrow\left\{\begin{array}{l}16.21 \\ 16.22\end{array}\right.$

Forgetting the vertical line in Figure 13.1, perturbing $A_{2}$ as shown in Figures 18.6 and 18.7, and perturbing $E_{8}$ as shown in Figures 19.1-19.4, we obtain the arrangements in Figure 16. $n$ for $n=3,4,8,9,10,11,17,18$ :

$$
18.6 \rightarrow(16.10,16.11,16.17,16.18), \quad 18.7 \rightarrow(16.3,16.4,16.8,16.9) .
$$

An algebraic realization of Figure 16.5 is shown in Figures 20.1-20.3.

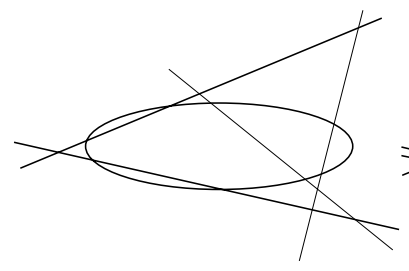

FigURE 20.1

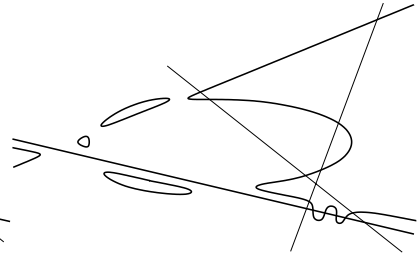

FiguRE 20.2

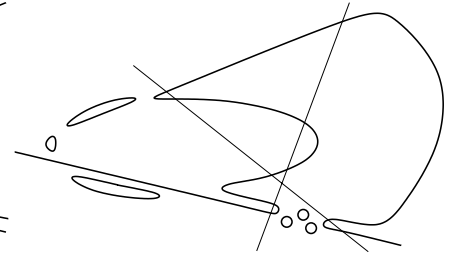

FiguRE 20.3

\section{§4. Algebraically unrealizable pseudoholomorphic arrangements OF A QUINTIC AND TWO LINES}

In this section we prove that the arrangements in Figures 21.1-21.3 and in Figure 16.12 are realizable by real pseudoholomorphic curves (see Subsection 4.5), but they are unrealizable by real algebraic curves (see Subsections 4.1-4.4). Moreover, in Subsection 4.5 we give a pseudoholomorphic realization of the two arrangements that are labeled by “尹*alg." in the list in Subsection 0.7.
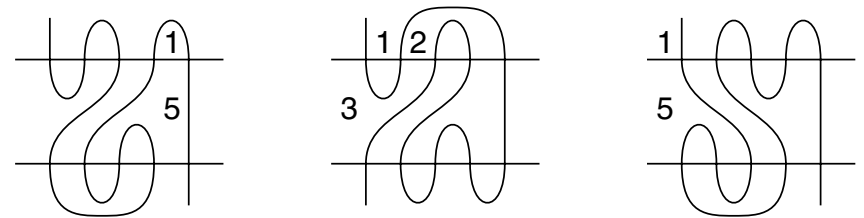

Figure 21.1.(3-2). Figure 21.2.(8-3). Figure 21.3.(11-1).

4.1. Algebraic unrealizability of the arrangement in Figure 21.1. Rotating $L_{1}$ around $q$ as shown in Figure 22.1 until the first tangency with the quintic, we obtain an arrangement as in Figure 22.2. Rotating $L_{2}$ around $q^{\prime}$ as shown in Figure 22.2, we obtain Figure 22.3 , where $\mathbb{R P}^{2}$ is depicted as a disk with opposite boundary points identified. The boundary of the disk represents $L_{1}$. Blowing up $p$, we obtain Figure 22.4, where the exceptional divisor is denoted by $P$. Blowing up $p^{\prime}$ and then blowing down $L_{1}$, we obtain a curve $C$ of bidegree $(4,8)$ on $\mathcal{F}_{2}$ (see Definition 2.10), depicted in Figure 22.5. This curve has a singular point of type $D_{4}$ (a simple triple point) on the line $P^{\prime}$. In Figure 22.5 , the fibers of $\mathbb{R} \mathcal{F}_{2} \rightarrow \mathbb{R P}^{1}$ correspond to vertical lines. 


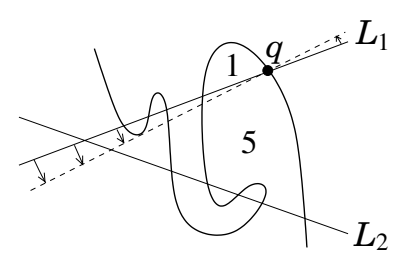

FiguRe 22.1

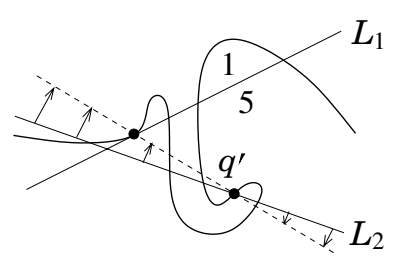

FIGURE 22.2

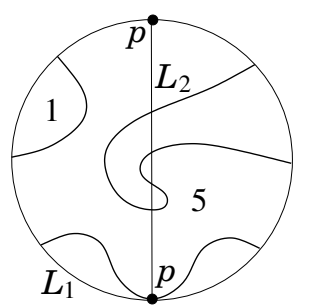

FIGURE 22.3

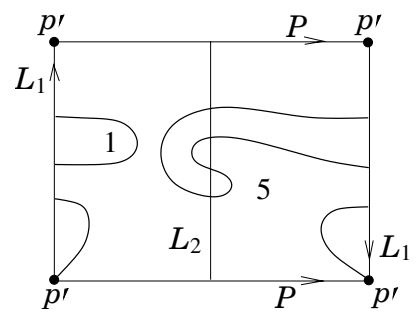

FiguRE 22.4

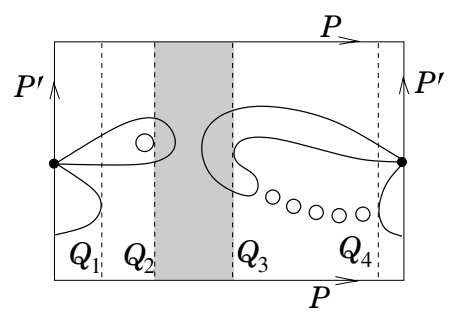

FIGURE 22.5

The end of the proof of the algebraic unrealizability of Figure 21.1 is similar to that in [22, §3]. Let $k$ be the self-linking number of $C$ on the interval of the pencil of vertical lines corresponding to the gray rectangle in Figure 22.5. Then the braid associated with the curve $C$ relative to the pencil of vertical lines has the form

$$
b=\sigma_{1}^{-1} \sigma_{2}^{-1} \sigma_{1} \sigma_{2}^{-1} \sigma_{1}^{-1} \sigma_{3}^{-1} \sigma_{2}^{-1} \sigma_{3}^{1+k} \sigma_{1}^{1-k} \sigma_{1}^{-6} \delta^{-1} \Delta^{2},
$$

where $\delta=\sigma_{3} \sigma_{2} \sigma_{3}$ is the part of the braid word corresponding to the singularity $D_{4}$ and $\Delta=\delta \sigma_{1} \sigma_{2} \sigma_{3}$ is the Garside element of the group of braids with 4 strings.

Lemma 4.1. If Figure 21.1 is pseudoholomorphically realizable, then $k=-3$.

Proof. The algebraic length of $b$ is zero. Hence, its quasipositivity is equivalent to its triviality. We show that $b$ is nontrivial for $k \neq-3$. Indeed, if $k$ is even, then $b$ determines a nontrivial permutation. If $k$ is odd, then the linking number of the second and the third string is equal to $k+3$.

Lemma 4.2. Suppose that a polynomial $P(y)=y^{4}+a_{2} y^{2}+a_{3} y+a_{4}$ has four real roots $y_{1}, \ldots, y_{4}$ such that $y_{1} \leq \cdots \leq y_{4}$. If $y_{1}+y_{4}<y_{2}+y_{3}$, then $a_{3}>0$. If $y_{1}+y_{4}>y_{2}+y_{3}$, then $a_{3}<0$.

Proof. We shall consider only the case where $y_{1}+y_{4}<y_{2}+y_{3}$. The opposite case is similar. We denote $\left(y_{2}+y_{3}\right) / 2$ by $b$. Since the coefficient of $y^{3}$ is zero, we have $y_{1}+\cdots+y_{4}=0$, whence $\left(y_{1}+y_{4}\right) / 2=-b$. Since $y_{1}+y_{4}<y_{2}+y_{3}$, we have $b>0$. Set $c=\left(y_{3}-y_{2}\right) / 2$ and $d=\left(y_{4}-y_{1}\right) / 2$. Then

$$
y_{1}=-b-d, \quad y_{2}=b-c, \quad y_{3}=b+c, \quad y_{4}=-b+d .
$$

Therefore,

$$
a_{3}=-y_{1} y_{2} y_{3}-y_{1} y_{2} y_{4}-y_{1} y_{3} y_{4}-y_{2} y_{3} y_{4}=2 b\left(d^{2}-c^{2}\right) .
$$

It remains to note that $d-c=y_{4}-y_{3}+2 b \geq 2 b>0$, and $c>0$.

Let $(x, y)$ be standard coordinates on $\mathcal{F}_{2}$ (see Definition 2.10). The equation of $C$ in the coordinates $(x, y)$ has the form

$$
y^{4}+a_{2}(x) y^{2}+a_{3}(x) y+a_{4}(x)=0, \quad \operatorname{deg}_{x} a_{m}(x)=2 m
$$


(as usual, we kill the coefficient of $y^{3}$ by the variable change $y \mapsto y-a_{1}(x) / 4$ ). Let $x=x_{0}, \ldots, x=x_{4}$ be the equations of the lines $P^{\prime}, Q_{1}, Q_{2}, Q_{3}, Q_{4}$, respectively (see Figure 22.5). As was shown in [22, Lemma 3.7], $a_{3}(x)$ has at least $2|k|-1$ roots in the segment $\left[x_{2}, x_{3}\right]$, and $k=-3$ by Lemma 4.1. By Lemma 4.2 we have $a_{3}\left(x_{0}\right)>0$, $a_{3}\left(x_{1}\right)<0$, and $a_{3}\left(x_{4}\right)>0$; hence, $a_{3}(x)$ has at least one root at each of the segments $\left[x_{0}, x_{1}\right]$ and $\left[x_{4}, x_{0}\right]$. Thus, $a_{3}(x)$ has at least $5+2=7$ roots. This contradicts the fact that $\operatorname{deg}_{x} a_{3}(x)=6$ (see Remark 4.4 in Subsection 4.4).

4.2. Algebraic unrealizability of the arrangement in Figure 21.2. Starting with Figure 21.2 and rotating the line around $p$ as shown in Figure 24, we obtain successively Figure 16.12 and Figure 16.5. The algebraic unrealizability of Figure 16.12 is proved in Subsection 4.4 (note that the arrangement in Figure 16.5 was realized algebraically at the end of $\S 3)$.

4.3. Algebraic unrealizability of the arrangement in Figure 21.3. Rotating $L_{1}$ around $q$ as shown in Figure 23, we transform Figure 21.3 into Figure 21.1, and the algebraic unrealizability of the latter was proved in Subsection 4.1.

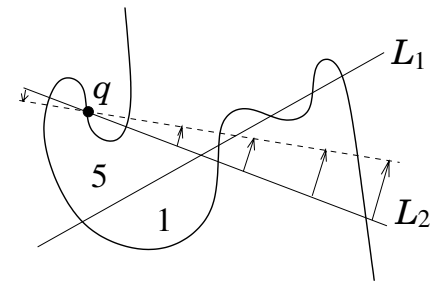

FigURE 23

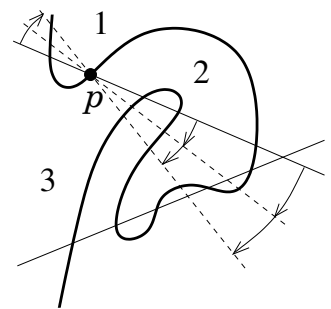

FigURE 24

4.4. Algebraic unrealizability of the arrangement in Figure 16.12. After preliminary manipulations depicted in Figures 25.1-25.5 and similar to those performed at the beginning of Subsection 4.1, we obtain an algebraic curve $C$ of bidegree $(4,8)$ on the Hirzebruch surface $\mathcal{F}_{2}$, depicted in Figure 25.5. This curve has a singularity of type $D_{4}$ (simple triple point). The three rightmost ovals appear in this order with respect to the

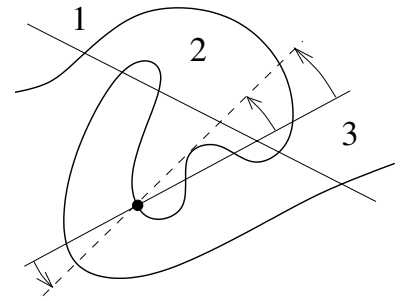

Figure 25.1

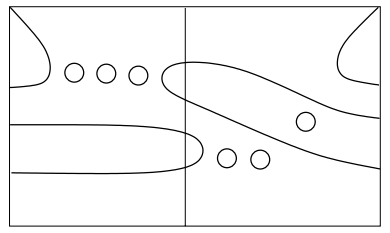

Figure 25.4

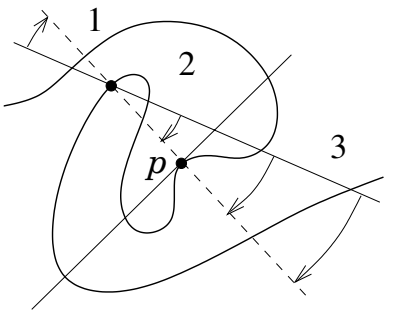

FiguRE 25.2

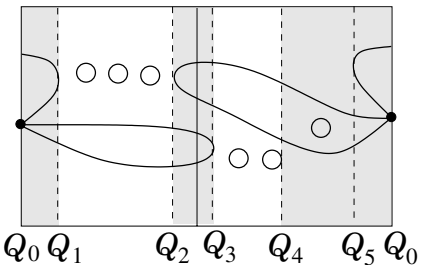

FiguRE 25.5

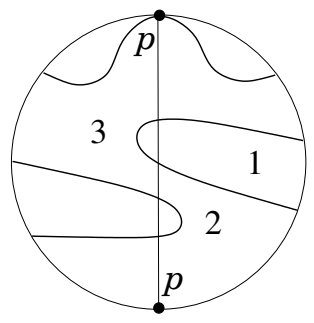

FiguRE 25.3

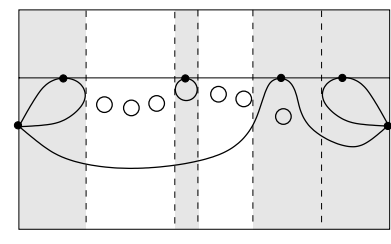

FiguRe 25.6 
fibers of $\mathbb{R} \mathcal{F}_{2} \rightarrow \mathbb{R P}^{1}$ (in Figure 25.5, the fibers correspond to vertical lines), because otherwise we would have too many real intersections of $C$ with an auxiliary line (i.e., with a curve of bidegree $(1,2))$ passing through the triple point and two of these ovals.

Let $R$ and $L$ be the cubic resolvent and the core of the curve $C$. These are curves of bidegrees $(3,12)$ and $(1,4)$, respectively, on $\mathcal{F}_{4}$. For their definitions and main properties, see [22, §3]. We only recall that all the intersections of $R$ and $L$ are tangencies, and that their $x$-coordinates (i.e., the projections to $\mathbb{P}^{1}$ ) are the roots of $a_{3}(x)$, where, as above, $y^{4}+a_{2}(x) y^{2}+a_{3}(x) y+a_{4}(x)$ is the defining polynomial of $C$ in some standard coordinates on $\mathcal{F}_{2}$.

It follows from 22, Lemma 3.3] that $R$ is arranged with respect to the fibers as in Figure 25.6, and that it lies beneath the core $L$ (in the sense of nonstrict inequalities). Moreover, Lemma 4.2 (see Subsection 4.1) implies that each interval corresponding to a gray rectangle bounded by dashed lines in Figure 25.5 contains an odd number of roots of $a_{3}(x)$. Hence, each gray rectangle in Figure 25.6 contains an odd number of tangency points of the curves $R$ and $L$. For the reason explained in Remark 4.5, it suffices to consider only the case where each gray rectangle contains a single tangency point and there are no other tangencies, i.e., where $R$ is arranged with respect to $L$ as in Figure 25.6.

Thus, the algebraic unrealizability of Figure 16.12 is a consequence of the following statement (take Remark 4.5 into account).

Proposition 4.3. Suppose that $R$ and $L$ are real algebraic curves on $\mathbb{R} \mathcal{F}_{4}$ of bidegrees $(3,12)$ and $(1,4)$, respectively, such that all (including nonreal) intersection points of $R$ and $L$ are tangency points and the curve $R$ has a singular point of type $D_{4}$. Then the mutual arrangement of $R$ and $L$ cannot be as in Figure 25.6.

Proof. The genus of $R$ is equal to 7 (the number of interior integral points in the triangle $(0,0)-(9,0)-(0,3))$. It follows that the curve $R$ is dividing; in particular, it has complex orientations.

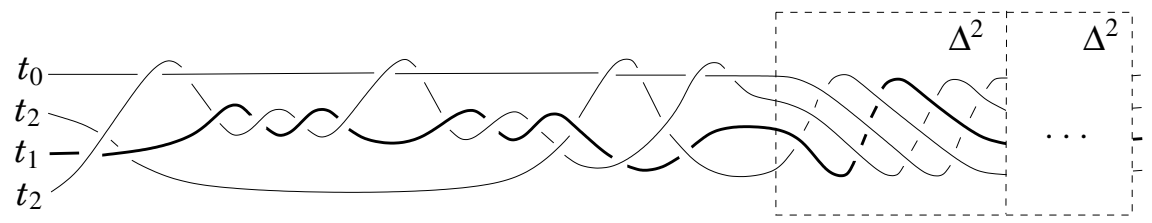

FiguRE 26

Let $b$ be the braid associated with the reducible curve $A=R \cup L$. We have

$$
b=\underbrace{\left(\sigma_{1}^{-1} \sigma_{2}^{-1} \sigma_{1}^{-1}\right)}_{D_{4}} \underbrace{\left(\sigma_{3}^{-2}\right)}_{A_{3}} \sigma_{1}^{-4} \underbrace{\left(\sigma_{3}^{-2}\right)}_{A_{3}} \sigma_{1}^{-3} \tau_{2,1} \underbrace{\left(\sigma_{3}^{-2}\right)}_{A_{3}} \sigma_{1}^{-1} \tau_{1,2} \underbrace{\left(\sigma_{3}^{-2}\right)}_{A_{3}} \Delta^{4},
$$

where $\tau_{2,1}=\sigma_{1}^{-1} \sigma_{2}=\tau_{1,2}^{-1}$ and $\Delta=\sigma_{1} \sigma_{2} \sigma_{3} \sigma_{1} \sigma_{2} \sigma_{1}$ (see Figure 26).

Let $\pi: \mathbb{C F}_{4} \rightarrow \mathbb{C P}^{1}$ be the standard projection. Let $H^{\circ}$ be one of the halves of $\mathbb{C P}^{1} \backslash \mathbb{R} \mathbb{P}^{1}$, namely, the half that induces the orientation of $\mathbb{R P}^{1}$ corresponding to the direction from the left to the right according to Figure 25.6. Let $H \subset H^{\circ}$ be a disk sufficiently close to $H^{\circ}$. Let $N=\pi^{-1}(H) \cap \mathbb{C} A$. Then the closure $\hat{b}$ of $b$ is the boundary of $N$. We put $N_{R}=N \cap \mathbb{C} R$ and $N_{0}=N \cap \mathbb{C} L$.

Computing the Euler characteristics of the components of $N$ and the linking numbers of the braid components, we see that $N_{R}$ has two connected components, one of which (denote it by $N_{1}$ ) is mapped bijectively onto $H$ and the other (denote it by $N_{2}$ ) is a double covering of $H$ branched at a single point. Moreover, $N_{1}$ is disjoint from $N_{0}$, and $N_{2}$ has a simple tangency with $N_{0}$ at a single point. We set $L_{j}=\partial N_{j}, j=0,1,2$. 
It follows that $b$ must have the form

$$
b=\left(a_{1} \sigma_{1} a_{1}^{-1}\right)\left(a_{2} \sigma_{1}^{4} a_{2}^{-1}\right) .
$$

Moreover, (11) can be refined as follows. Let $\hat{B}_{1,1,2}=\hat{B}_{1,1,2}\left(t_{0}, t_{1}, t_{2}\right)$ be the groupoid of colored braids introduced in [15. We color the strings of $b$ in the colors $t_{0}, t_{1}, t_{2}$ in accordance with the decomposition $\hat{b}=L_{0} \sqcup L_{1} \sqcup L_{2}$. Then $b \in G=\operatorname{Aut}\left(t_{2}, t_{1}, t_{2}, t_{0}\right)$, and the above description of the mutual arrangement of the surfaces $N_{0}, N_{1}, N_{2}$ means that $b$ has the form $b=b_{1} b_{4}$, where the colored braid $b_{1}$ is a conjugate of

$$
\sigma_{1}:\left(t_{2}, t_{2}, t_{0}, t_{1}\right) \rightarrow\left(t_{2}, t_{2}, t_{0}, t_{1}\right)
$$

(i.e., of a braid $\sigma_{1}$ whose first two strings are colored in the color $t_{2}$ ), and $b_{4}$ is a conjugate of

$$
\sigma_{1}^{4}:\left(t_{0}, t_{1}, t_{2}, t_{2}\right) \rightarrow\left(t_{0}, t_{1}, t_{2}, t_{2}\right) .
$$

We show that this is impossible for the braid (10). Let $\rho$ be the Burau representation of $\hat{B}_{1,1,2}$, described in [15, with the values of the parameters $t_{0}=t_{2}=-\tau, t_{1}=\tau^{2}$, where $\tau=(-1+i \sqrt{3}) / 2=\exp (2 \pi i / 3)$. Then the eigenvalues of $\rho\left(b_{4}\right)$ are $\left(1,1, t_{0}^{2} t_{1}^{2}\right)=$ $\left(1,1, \tau^{6}\right)=(1,1,1)$. Moreover, it is easy to check that $\rho\left(b_{4}\right)$ is the identity matrix. Thus, $b=b_{1} b_{4}$ implies $\rho(b)=\rho\left(b_{1}\right)$. The latter identity is impossible because the eigenvalues of $\rho\left(b_{1}\right)$ must be $\left(1,1,-t_{0}\right)=(1,1, \tau)$, whereas it is easy to compute that the eigenvalues of $\rho(b)$ are $\left(\alpha, \tau \alpha, \tau^{2} \alpha\right)$, where $\alpha^{3}=\tau$. To compute $\rho(b)$ (especially, by hand), it is convenient to use the fact that $\rho\left(\Delta^{2}\right)$ is a scalar matrix for any choice of parameters (see also Remark 4.7 below).

Remark 4.4. In terms of cubic resolvents, the proof of the algebraic unrealizability of Figure 21.1 given in Subsection 4.1 means the following. The topological properties of the curve in Figure 22.5 show that $R$ and $L$ have at least $(2|k|-1)+2=7$ real tangency points. This contradicts the fact that the intersection number of these divisors on $\mathcal{F}_{4}$ is equal to 12 .

Remark 4.5. Lemma 4.2 allows us to find only the parity of the number of roots of $a_{3}(x)$ on every interval bounded by vertical tangents to $C$. However, when writing the braid word, each time we choose the minimal possible values of these numbers, which are equal to 0 or 1 . What happens if we increase some value by 2? Clearly, in this case two successive tangency points appear on the corresponding interval. This means that the subword $\sigma_{3}^{-4}$ is inserted somewhere into the braid word of $b$. Thus, this does not affect the subsequent arguments.

Remark 4.7. One can use the formulas given in [15] for the Burau matrices of colored braids. However, the computation becomes easier if the base is changed (see Remark $4.8)$ as explained below. For example, $(3 \times 3)$-matrices are needed in this case rather than $(4 \times 4)$-matrices. Let

$$
b=\sigma_{j_{1}}^{\varepsilon_{1}} \ldots \sigma_{j_{n}}^{\varepsilon_{n}}, \quad \varepsilon_{\nu} \in\{-1,+1\},
$$

be a braid with $m$ strings colored in the colors $t_{i_{1}}, \ldots, t_{i_{m}}$ along the left-hand side, and hence, in the colors $t_{i_{\pi(1)}}, \ldots, t_{i_{\pi(m)}}$ along the right-hand side, where $\pi$ is the permutation determined by $b$. Then

$$
\rho(b)=S_{j_{1}}\left(t_{k_{1}}\right)^{\varepsilon_{1}} \ldots S_{j_{n}}\left(t_{k_{n}}\right)^{\varepsilon_{n}},
$$

where $t_{k_{\nu}}$ is the color of the lower string (in the sense of over-/under-passes) at the $\nu$ th crossing, i.e.,

$$
k_{\nu}= \begin{cases}\pi_{\nu-1}^{-1}\left(j_{\nu}\right)=\pi_{\nu}^{-1}\left(j_{\nu}+1\right), & \varepsilon_{\nu}=+1, \\ \pi_{\nu-1}^{-1}\left(j_{\nu}+1\right)=\pi_{\nu}^{-1}\left(j_{\nu}\right), & \varepsilon_{\nu}=-1\end{cases}
$$


(here $\pi_{\nu}$ denotes the permutation determined by $\left.\sigma_{j_{1}}^{\varepsilon_{1}} \ldots \sigma_{j_{\nu}}^{\varepsilon_{\nu}}\right)$, and $S_{j}(t)$ is the $((m-1) \times$ $(m-1))$-matrix obtained by deleting the first and the last rows and columns from the $((m+1) \times(m+1))$-matrix

$$
I_{j-1} \oplus\left(\begin{array}{ccc}
1 & 0 & 0 \\
t & -t & 1 \\
0 & 0 & 1
\end{array}\right) \oplus I_{m-j-1}
$$

here $I_{p}$ is the identity $(p \times p)$-matrix and $A \oplus B$ is the block-diagonal matrix $\left(\begin{array}{cc}A & 0 \\ 0 & B\end{array}\right)$.

For example, to check the proof of Proposition 4.1, it suffices to do the following matrix computation. Set $S_{j}=S_{j}\left(t_{0}\right)=S_{j}\left(t_{2}\right)=S_{j}(-\tau)$ and $T_{j}=S_{j}\left(t_{1}\right)=S_{j}\left(\tau^{2}\right)$, i.e.,

$$
\begin{array}{cc}
S_{1}=\left(\begin{array}{lll}
\tau & 1 & 0 \\
0 & 1 & 0 \\
0 & 0 & 1
\end{array}\right), \quad S_{2}=\left(\begin{array}{ccc}
1 & 0 & 0 \\
-\tau & \tau & 1 \\
0 & 0 & 1
\end{array}\right), \quad S_{3}=\left(\begin{array}{ccc}
1 & 0 & 0 \\
0 & 1 & 0 \\
0 & -\tau & \tau
\end{array}\right), \\
T_{1}=\left(\begin{array}{ccc}
-\tau^{2} & 1 & 0 \\
0 & 1 & 0 \\
0 & 0 & 1
\end{array}\right), \quad T_{2}=\left(\begin{array}{ccc}
1 & 0 & 0 \\
\tau^{2} & -\tau^{2} & 1 \\
0 & 0 & 1
\end{array}\right), \quad T_{3}=\left(\begin{array}{ccc}
1 & 0 & 0 \\
0 & 1 & 0 \\
0 & \tau^{2} & -\tau^{2}
\end{array}\right) .
\end{array}
$$

Then (see Figure 26)

$$
\begin{aligned}
\rho(b)= & T_{1}^{-1} S_{2}^{-1} S_{1}^{-1} S_{3}^{-2}\left(S_{2}^{-1} T_{2}^{-1}\right)^{2} S_{3}^{-2}\left(S_{2}^{-1} T_{2}^{-1} S_{2}^{-1}\right) S_{1}^{-1} S_{2} T_{1}^{-1} S_{3}^{-2} S_{2}^{-1} T_{1} S_{3}^{-2} \\
& \times\left(S_{1} S_{2} S_{3} T_{1} T_{2} T_{3}\left(S_{1} S_{2} S_{3}\right)^{2}\right)^{2} \\
= & \left(\begin{array}{ccc}
-1 & \tau & 1 \\
\tau & -\tau^{2} & -2 \tau \\
-\tau^{2} & 0 & -\tau
\end{array}\right), \\
& \rho\left(b_{1}\right) \sim S_{1}, \quad \text { and } \quad \rho\left(b_{4}\right) \sim S_{1} T_{1} S_{1} T_{1}=I .
\end{aligned}
$$

In order to compute $\rho(b)$ faster, the following identities can be used (recall that $\tau^{3}=$ 1 and $\left.\tau^{2}=-\tau-1\right):\left(S_{2}^{-1} T_{2}^{-1}\right)^{2}=I$ (hence, also $\left.S_{2}^{-1} T_{2}^{-1} S_{2}^{-1}=T_{2}\right)$ and $\rho\left(\Delta^{2}\right)=$ $S_{1} S_{2} S_{3} T_{1} T_{2} T_{3}\left(S_{1} S_{2} S_{3}\right)^{2}=-\tau^{2} I$.

Remark 4.8. When talking in the previous remark about the change of the base in the Burau representation described in [15, we were not quite rigorous. Recall that a groupoid is a category such that all its morphisms are invertible and its representation is a functor to the category of modules over some ring. The Burau representation was defined in 15 as a functor that associates the same free module $V$ with every object of the groupoid of colored braids (i.e., with every permutation of the colors). The matrices given in [15] are the matrices of the images of morphisms with respect to a fixed base of $V$. Actually, there is no base of $V$ such that the matrices of the images of braids take the form described in Remark 4.7. However, it is possible to associate different copies of $V$ with different objects, and to find suitable bases in each copy so that the matrices become as in Remark 4.7 .

\subsection{Construction of pseudoholomorphic arrangements.}

Proposition 4.9. The arrangements in Figures 21.1-21.3, in Figure 16.12, and in Figures 27.1-27.2 are realizable by a real pseudoholomorphic quintic and two lines. 

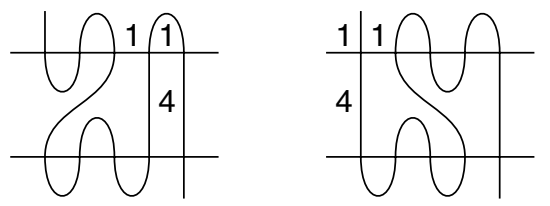

Figure 27.1. (6-7). Figure 27.2. (18-5).

Proof. It is easy to check that the braid $b$ defined in Subsection 4.1 is trivial for $k=-3$. Indeed,

$$
\begin{aligned}
& b^{-1}=\Delta^{-2} \delta \sigma_{1}^{2} \sigma_{3}^{2} \sigma_{2} \sigma_{3} \sigma_{1} \sigma_{2} \sigma_{1}^{-1} \sigma_{2} \sigma_{1}=\Delta^{-2} \delta \sigma_{1} \sigma_{3}(\overbrace{\sigma_{1} \sigma_{3} \sigma_{2} \sigma_{3} \sigma_{1} \sigma_{2}}^{\Delta}) \sigma_{2} \sigma_{1} \sigma_{2}^{-1} \\
& =\left(\Delta^{-2} \sigma_{2}\right)\left(\sigma_{3} \sigma_{2} \sigma_{1} \sigma_{3} \Delta\right) \sigma_{2} \sigma_{1} \sigma_{2}^{-1}=\left(\sigma_{2} \Delta^{-2}\right)(\underbrace{\left.\sigma_{1} \sigma_{2} \sigma_{3} \sigma_{1}\right) \sigma_{2} \sigma_{1}}_{\Delta} \sigma_{2}^{-1}=1 .
\end{aligned}
$$

It follows that Figure 21.1 is pseudoholomorphically realizable. The pseudoholomorphic realizability of Figure 21.3 is equivalent to that of Figure 21.1 because the braid associated with the pencil of lines centered at $q$ (see Figure 23) is the same in both cases.

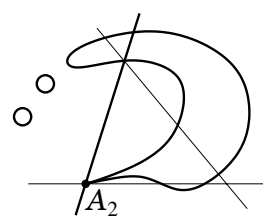

FiguRE 28.1

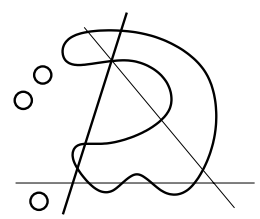

FigURE 28.2

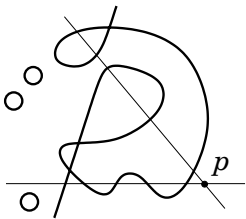

FiguRE 28.3

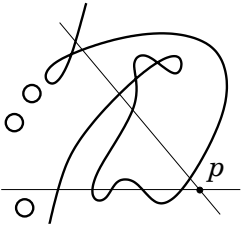

FIGURE 28.4

To prove that Figure 21.2 is pseudoholomorphically realizable, I could merely write down a quasipositive decomposition of the corresponding braid, leaving it to the reader to check the identity in the braid group. Instead, here I give a geometric proof that does not require any computation. It is depicted in Figures 28.1-28.4. By a small perturbation of a double conic, it is easy to construct an algebraic cuspidal quartic arranged as in Figure 28.1 with respect to three lines. Then, by successive perturbations, we can obtain Figure 28.2 and Figure 28.3 (still remaining in the class of algebraic curves). Next, the pseudoholomorphic realizability of Figure 28.3 implies that of Figure 28.4, because they determine the same braid with respect to the pencil of lines centered at $p$. Finally, a perturbation of double points of the quintic in Figure 28.4 yields Figure 21.2.

The pseudoholomorphic realizations of Figures 16.5 and 16.12 can be obtained from Figure 21.2 by rotation of one of the lines as shown in Figure 24 (see Subsection 4.2).

Finally, to realize Figures 27.1-27.2 pseudoholomorphically, note that the above braid $b$ corresponds not only to the curve depicted in Figure 22.5, but also to the curve obtained from it by removing one of the five ovals situated between $Q_{3}$ and $Q_{4}$, and by replacing the three lower intersection points on $Q_{3}$ with a singularity of type $A_{2}$ tangent to $Q_{3}$. Perturbing this singularity, we arrive at Figure 27.1, and Figure 27.2 can be obtained from it.

\section{§5. Degeneration of a conic into a pair of lines}

Let $O$ be a simple closed curve dividing $\mathbb{R P}^{2}$ into a disk $D$ and a Möbius band $M$. Let $C$ be a smooth real curve on $\mathbb{R P}^{2}$, let $a$ and $b$ be two points on $O$ not lying on $C$, and let $\gamma$ be one of the two arcs into which $a$ and $b$ divide $O$. We say that $\gamma$ is minimal with respect to $C$ inside $O$ (respectively, outside $O$ ) if any path $\gamma^{\prime}$ from $a$ to $b$ contained 
in $D$ (respectively, contained in $M$ and homotopic in $M$ to $\gamma$ ) has at least as many intersections with $C$ as $\gamma$.

Proposition 5.1. Let $C_{2}$ and $C_{n}$ be nonsingular real algebraic curves on $\mathbb{R P}^{2}$ of degrees 2 and $n$, respectively, that have $2 n$ real intersections. Suppose that the following condition is fulfilled.

$(*)$ There exist points $a, b, c, d$ lying on $C_{2}$ in this cyclic order such that the arcs ab and $c d$ are minimal with respect to $C_{n}$ inside $C_{2}$, and the arcs bc and da are minimal with respect to $C_{n}$ outside $C_{2}$.

Then the mutual arrangement of $C_{2}$ and $C_{5}$ is isotopic to the mutual arrangement of $C_{2}^{\prime}$ and $C_{5}$, where $C_{2}^{\prime}$ is a smooth perturbation of a union of two lines each of which has $n$ real intersection points with the curve $C_{n}$.

The same statement is true for real pseudoholomorphic curves.

Proof. Consider the pencil of conics through $a, b, c, d$.

Let $C_{2}$ and $C_{5}$ be a conic and an $M$-quintic that have 10 real intersection points, all of them lying on the odd branch $J_{5}$ of $C_{5}$. Recall that the notion of passage through infinity was defined in Subsection 0.4, and a nest of arcs inside (outside) an oval was defined in Subsection 0.1 before Theorem 3 .

Corollary 5.2. Suppose that one of the following conditions is fulfilled:

(1) there are at least three passages through infinity;

(2) there is a nest of depth 5 inside $C_{2}$ formed by arcs of $C_{5}$;

(3) there are two disjoint nests outside $C_{2}$ formed by arcs of $C_{5}$;

(4) $J_{5}$ and $C_{2}$ are arranged as in one of Figures 29.1-29.2 (the series 16, 18 in Subsection 0.5).

Then condition $(*)$ is satisfied, and hence $C_{5} \cup C_{2}$ degenerates into a quintic and two lines.

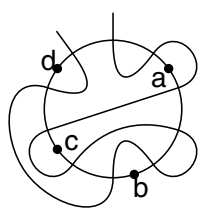

FiguRE 29.1

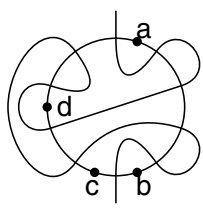

FiguRE 29.2

Proof. In each of the cases (1)-(4), we describe a choice of $a, b, c, d$ ensuring (*). Let $D$ and $M$ be the interior and the exterior components of $\mathbb{R P}^{2} \backslash C_{2}$.

(1) There are five arcs of $C_{5}$ outside $C_{2}$ with ends on $C_{2}$. If all of them pass through infinity (i.e., are nontrivial in $H_{1}\left(M, C_{2}\right)$ ), then $\left(^{*}\right)$ is true for any choice of $a, b, c, d$ such that the arcs $a b$ and $c d$ do not meet $C_{5}$. Otherwise, there exist two $\operatorname{arcs} \alpha_{1}, \alpha_{2}$ of $C_{5}$ that are trivial in $H_{1}\left(M, C_{2}\right)$. Let $D_{j}$ be the disk cut from $M$ by $\alpha_{j}$, and let $\beta_{j}=C_{2} \cap\left(\partial D_{j}\right)$. Choose $a, b \in \beta_{1}$ and $c, d \in \beta_{2}$.

(2) Let $D_{1} \subset \cdots \subset D_{5}$ be the disks involved in the definition of a nest, i.e., such that $D \cap\left(\partial D_{j}\right) \subset C_{5}, j=1, \ldots, 5$. Choose $b, c \in C_{2} \cap D_{1}$ and $a, d \in C_{2} \backslash D_{5}$.

(3) Let $\alpha_{1}, \ldots, \alpha_{4}$ be the exterior arcs of $C_{5}$ forming the nests, and let $D_{j}$ be the disk cut from $M$ by $\alpha_{j}$. Set $\beta_{j}=C_{2} \cap\left(\partial D_{j}\right)$. Let $D_{1} \subset D_{2}$ and $D_{3} \subset D_{4}$. The Bézout theorem for an auxiliary line shows that no nest of depth three is possible; hence, $D_{2} \cap D_{4}=\varnothing$. Choose $a, b \in \beta_{1}$ and $c, d \in \beta_{3}$.

(4) Choose $a, b, c, d$ as shown in Figures 29.1-29.2. 
Thus, under one of conditions (1)-(4) of Corollary 5.2, an arrangement is realizable by real pseudoholomorphic (respectively, algebraic) curves if and only if it can be obtained as a perturbation of the union of a quintic and two lines.

\section{$\S 6$. Classification of arrangements of $C_{5} \cup C_{2}$ Of Series NOT COVERED BY COROLlARY 5.2}

6.1. Enumeration of arrangements of $J_{5} \cup C_{2}$ to be considered. Now we consider the arrangements of $C_{2} \cup J_{5}$ that do not satisfy conditions (1)-(4) of Corollary 5.2, because it is natural to postpone the classification of the others until the classification of $C_{5} \cup L_{1} \cup L_{2}$ is finished. Moreover, it is not necessary to consider arrangements that contradict the Bézout theorem for auxiliary lines, for instance, those having a nest of exterior $\operatorname{arcs}$ of $J_{5}$ of depth greater than two. Up to symmetry, there are 24 such arrangements. These are the series $\mathbf{1 - 1 5}, \mathbf{1 7}, \mathbf{1 9}, \mathbf{2 2 - 2 5}, \mathbf{2 9}, \mathbf{3 2}, \mathbf{3 3}$ in the list in Subsection 0.5.

It is not difficult to check this directly, but this can also be seen from the list [7 of all arrangements of $J_{5} \cup C_{2}$ with one passage through infinity that do not contradict Bézout's theorem. For the reader's convenience, in the following table we give the correspondence between the numbers of series in Subsection $0.5(\mathbf{1}, \mathbf{2}, \ldots)$ and in $[7](M 1, M 2, \ldots)$ :

\begin{tabular}{|c|c|c|c|c|c|c|c|c|c|c|c|}
\hline 1 & M30 & 12 & $M 21$ & 23 & $M 2$ & $M 1$ & 9 & M12 & 20 & $M 23$ & 33 \\
\hline 2 & M33 & 13 & M3 & 24 & $M 20$ & $M 2$ & 23 & $M 13$ & 21 & $M 24$ & 6 \\
\hline 3 & $M 18$ & 14 & $M 6$ & 25 & $M 10$ & M3 & 13 & $M 14$ & 26 & $M 25$ & 19 \\
\hline 4 & $M 28$ & 15 & $M 26$ & 26 & $M 14$ & $M 4$ & 17 & $M 15$ & 30 & $M 26$ & 15 \\
\hline 5 & M32 & 16 & $M 27$ & 27 & $M 16$ & M5 & 28 & $M 16$ & 27 & $M 27$ & 16 \\
\hline 6 & $M 24$ & 17 & $M 4$ & 28 & M5 & M6 & 14 & $M 17$ & 29 & $M 28$ & 4 \\
\hline 7 & M31 & 18 & M9 & 29 & $M 17$ & $M 7$ & 10 & $M 18$ & 3 & M29 & 8 \\
\hline 8 & $M 29$ & 19 & $M 25$ & 30 & $M 15$ & $M 8$ & 22 & $M 19$ & 11 & M30 & 1 \\
\hline 9 & $M 1$ & 20 & $M 12$ & 31 & $M 11$ & $M 9$ & 18 & M20 & 24 & M31 & 7 \\
\hline 10 & $M 7$ & 21 & $M 13$ & 32 & $M 22$ & $M 10$ & 25 & M21 & 12 & M32 & 5 \\
\hline 11 & M19 & 22 & $M 8$ & 33 & $M 23$ & M11 & 31 & $M 22$ & 32 & M33 & 2 \\
\hline
\end{tabular}

6.2. Arrangements with nested exterior arcs. Let $C_{2}$ and $C_{5}$ be a conic and an $M$-quintic such that the odd branch of $J_{5}$ cuts $C_{2}$ at ten distinct points. Let $D$ (disk) and $M$ (Möbius band) be the components of $\mathbb{R P}^{2} \backslash C$. Suppose that there is a nest outside $C_{2}$ formed by arcs of $J_{5}$; i.e., there exist $\operatorname{arcs} \alpha_{1}$ and $\alpha_{2}$ which cut disks $D_{1}$ and $D_{2}$ from $M$ such that $D_{1} \subset D_{2}$. We choose $p \in D_{1} \cap C_{2}$ and denote the pencil of lines through $p$ by $\mathcal{L}_{p}$. Let $L_{\infty} \in \mathcal{L}_{p}$ be the tangent to $C_{2}$ at $p$. Let $(x, y)$ be affine coordinates $(x, y)$ such that $L_{\infty}$ is the infinite line, $\mathcal{L}_{p}$ is the pencil of vertical lines $x=$ const, and $C_{2}$ is the parabola $y=x^{2}$.

We shall use the encoding of the arrangement of $C=C_{5} \cup C_{2}$ with respect to $\mathcal{L}_{p}$ (fiberwise arrangements) proposed in [13] (and used in [14, 16, 20, 9] and in §3). Namely, we shall encode such an arrangement by a word composed of the symbols $\subset_{k}, \supset_{k}, \times_{k}$, which denote (respectively) a point of minimum and maximum of the $x$-coordinate and a double point on $C$. In all three cases, $k$ is the height of the point; i.e., the vertical line through this point has $k-1$ transversal intersections with $C$, with a smaller $y$-coordinate. We abbreviate a subword $\subset_{k} \supset_{k}$ to $o_{k}$.

The braid corresponding to a fiberwise arrangement of $C$ has the form $b=b_{\mathbb{R}} b_{\infty}$, where $b_{\mathbb{R}}$ is computed from the encoding word by the rules formulated in 13 for the case where $p \notin C$, but $b_{\infty}=\Delta \sigma_{1} \sigma_{2} \ldots \sigma_{6}$ (in place of $b_{\infty}=\Delta$, as it is for $p \notin C$ ).

By the above assumption, we need to consider only the series listed in the first two columns of Table 1 . Then, up to zigzag removing (deleting subwords of the form $\subset_{j} \supset_{j \pm 1}$ ), 
the arrangement of $C$ with respect to $\mathcal{L}_{p}$ is encoded by the word in the third column of Table 1, where the variables $u_{1}, u_{2}, u_{3}$ should be replaced by sequences of the form $o_{i_{1}} o_{i_{2}} \ldots$ of total length 6 .

If we drop $C_{2}$, what remains is a quintic whose fiberwise encoding with respect to $\mathcal{L}_{p}$ has the form either $\left[\supset_{3} o_{i_{1}} \ldots o_{i_{6}} \subset_{4}\right]$ or $\left[\supset_{3} o_{i_{1}} \ldots o_{i_{k}} \subset_{3} \supset_{4} o_{i_{k+1}} \ldots o_{i_{6}} \subset \subset_{4}\right]$, where the indices $i_{1}, \ldots, i_{k}$ are computed starting with the encoding word for $C$ by using evident rules.

Among all words of this form, only the following can encode a fiberwise arrangement of an $M$-quintic:

$$
\left[\supset_{3} o_{2}^{6} \subset_{4}\right], \quad\left[\supset_{3} o_{3}^{4} o_{2}^{2} \subset_{4}\right], \quad\left[\supset_{3} o_{3}^{4} o_{4}^{2} \subset_{4}\right], \quad\left[\supset_{3} o_{4}^{6} \subset_{4}\right]
$$

and

$\left[\supset_{3} o_{3}^{4} O_{2} \subset_{3} \supset_{4} O_{2} \subset_{4}\right],\left[\supset_{3} o_{2}^{5} \subset_{3} \supset_{4} O_{2} \subset_{4}\right],\left[\supset_{3} o_{3}^{3} \subset_{3} \supset_{4} o_{3} o_{4}^{2} \subset_{4}\right],\left[\begin{array}{l}\supset_{3} o_{4} \subset_{3} \supset_{4} o_{4}^{5} \subset_{4}\end{array}\right]$.

In the former case, this easily follows from the classification of affine quintics and from Bézout's theorem for auxiliary lines. In the latter case, this was proved in $\S 3$.

Hence, it suffices to consider only those values of the parameters in the words in Table 1 that provide one of these eight words after dropping the conic. Moreover, since $\times_{3}$ commutes with $o_{j}$ for $j \neq 4$, in the words containing subwords of the form $u_{\nu-1} \times{ }_{3}^{\alpha} u_{\nu}$, it suffices to consider only the cases where $u_{\nu}$ either is empty or begins with $o_{4}$.

For all such words, I checked with a computer if the Murasugi-Tristram inequality for the usual signature is fulfilled (see the details in [13, 16]). All the cases where it is fulfilled are listed in the last column of Table 1, where an expression of the form $\left[i_{1} \ldots i_{k}\right]\left[i_{k+1} \ldots i_{m}\right] \ldots$ means that $u_{1}=o_{i_{1}} \ldots o_{i_{k}}, u_{2}=o_{i_{k+1}} \ldots o_{i_{m}}, \ldots ;$ moreover, if the parameters $\alpha$ and $\beta=5-\alpha, 3-\alpha$, or $2-\alpha$ occur in the encoding word, then their values, if they are needed, are indicated as lower indices between the brackets.

\section{TABLE 1}

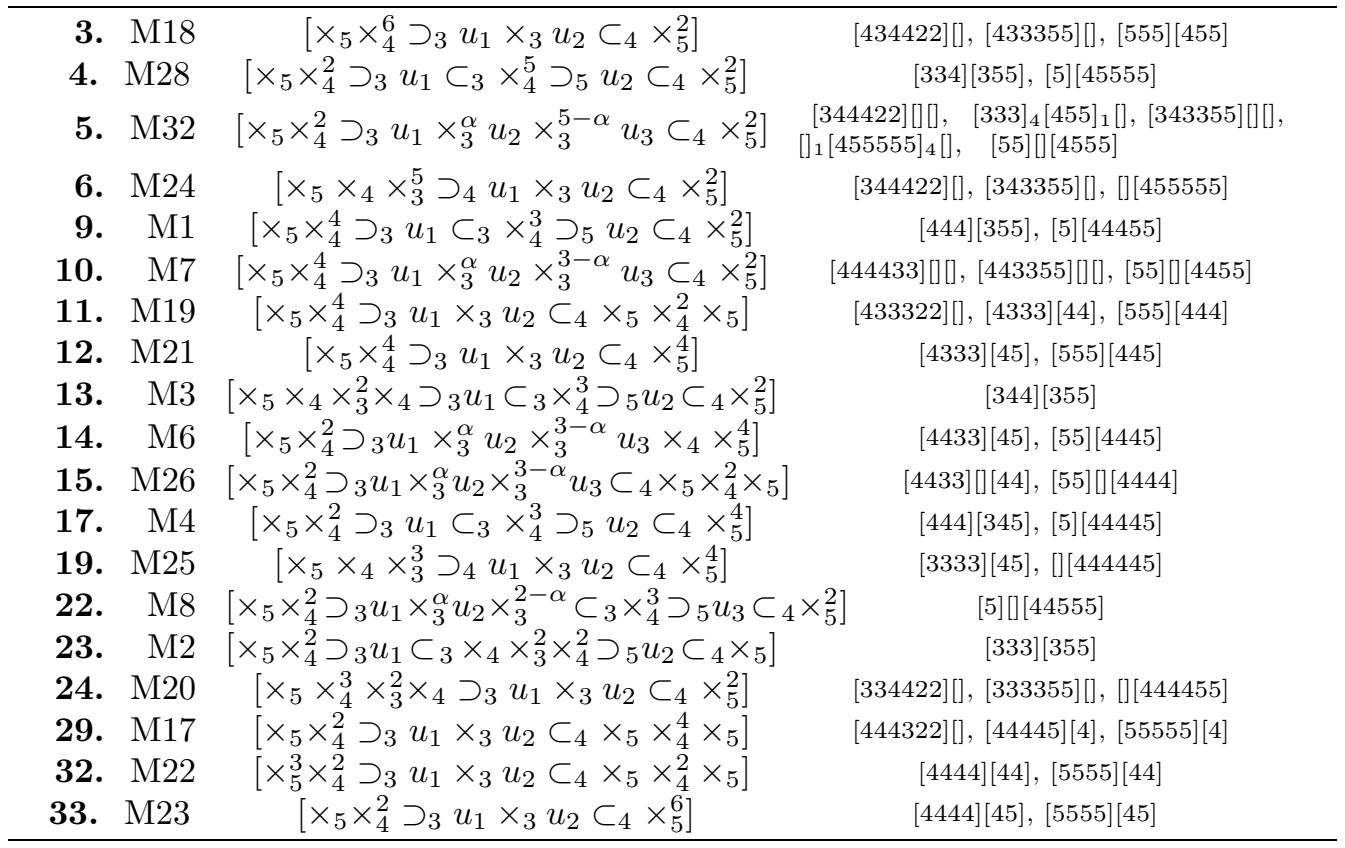

It remains to check that the parameter values listed in the last column provide only the arrangements listed in Subsection 0.5. 
6.3. Zigzag removal and fiberwise models. Let $(x, y)$ be an affine coordinate system on $\mathbb{R P}^{2}$, and let $p$ be the infinite point on the $y$-axis. Let $\mathcal{L}_{p}$ be the pencil of lines centered at $p$, i.e., the pencil of vertical lines $x=$ const. Let $A$ be a nodal real pseudoholomorphic curve of degree $m$, not passing through $p$, and let $\mathbb{R} A$ be the set of its real points. After an arbitrarily small shift of $p$, we may assume that $A$ is in general position with respect to $\mathcal{L}_{p}$; i.e., any line in $\mathcal{L}_{p}$ neither is tangent to $\mathbb{R} A$ at a flex point or at a double point, nor passes through two critical points (by a critical point we mean either a double point, or a point with vertical tangent).

We define a fiberwise isotopy with respect to the pencil of lines $\mathcal{L}_{p}$ (or simply fiberwise isotopy if it is clear what pencil is meant) to be an isotopy of $\mathbb{R} \mathbb{P}^{2}$ such that the image of any line in $\mathcal{L}_{p}$ at any moment is a line (maybe, another) belonging to the same pencil. By a fiberwise arrangement of a curve with respect to $\mathcal{L}_{p}$ (or simply fiberwise arrangement) we mean the equivalence class of a curve under fiberwise isotopies. As above, we shall encode the fiberwise arrangements by symbols $\subset_{k}, \supset_{k}, \times_{k}, o_{k}$.

We say that a real nodal curve is in almost general position with respect to $\mathcal{L}_{p}$ if all the above general position conditions are fulfilled with the only exception: one of the branches at a double point may have a vertical tangent, but this branch may not have a flex at the double point. To encode such points, we shall use the symbols $\in_{k}$ and $\ni_{k}$ (a perturbation of $\epsilon_{k}$ yields $\subset_{k} \times_{k+1}$ or $\subset_{k+1} \times_{k}$ ).

A smooth family $\left\{B_{t}\right\}_{t \in[0,1]}$ of immersed curves not passing through $p$ will be called an admissible isotopy with respect to $\mathcal{L}_{p}$ if there exists a finite set $\left.T=T^{\prime} \cup T_{\text {flex }} \subset\right] 0,1[$ such that:

(i) if $t \notin T \cup\{0,1\}$, then the curve $B_{t}$ is in general position with respect to $\mathcal{L}_{p}$;

(ii) if $t=0$ or 1 , then the curve $B_{t}$ is in almost general position with respect to $\mathcal{L}_{p}$;

(iii) if $t \in T$, then the general position conditions are fulfilled with the following two exceptions: 1) for $t \in T^{\prime}$, there exists a unique vertical line passing through exactly two critical points at least one of which is a double point; 2) for $t \in T_{\text {flex }}$, there exists a unique flex point with vertical tangent, and the number of critical points decreases when $t$ passes through such a value.

A passage through $t \in T_{\text {flex }}$ will be called a zigzag removal. It is easily seen that during an admissible isotopy the minimal number of intersections with vertical lines is constant, and the maximal number does not increase.

We define a fiberwise model of a curve $A$ as a set $B$ such that there exists an admissible isotopy $\left\{B_{t}\right\}$ with $B_{0}=\mathbb{R} A$ and $B_{1}=B$.

We say that a union of immersed circles $B$ satisfies the $k$-condition with respect to $p$ if any line in $\mathcal{L}_{p}$ meets $B$ at $k$ points at least. We say that $B$ satisfies the strong $k$-condition if the image of $B$ under any diffeomorphism of the pair $\left(\mathbb{R P}^{2}, p\right)$ onto itself satisfies the $k$-condition. If a curve $B$ satisfies the $(m-2)$-condition and any line of $\mathcal{L}_{p}$ meets it at $m$ points at most, then this curve uniquely determines an $m$-braid (denoted by $\operatorname{br}(B)$ ) such that $B=\mathbb{R} A$ for some real pseudoholomorphic curve $A$ if and only if the braid $\operatorname{br}(B)$ is quasipositive, i.e., can be written in the form $\prod_{j} a_{j} \sigma_{i_{j}} a_{j}^{-1}$ (see the details in [13, 16]).

Using this terminology, we can reformulate the statement on zigzag removal [16, Proposition 2.2] (more precisely, one of its corollaries) as follows.

Proposition 6.1. Let $A$ be a nodal real pseudoholomorphic curve in $\mathbb{R P}^{2}$ of degree $m$, in general position with respect to $p \in \mathbb{R P}^{2}$, and such that $\mathbb{R} A$ satisfies the $(m-2)$-condition. Then for any fiberwise model $B$ of $A$, the braid $\operatorname{br}(B)$ is quasipositive.

In all my previous papers on applications of quasipositive braids to problems of real geometry (including the previous section of this paper), the following statement or its analogs for other contexts was used implicitly. 
Proposition 6.2. Let $A$ be a nodal real pseudoholomorphic curve in $\mathbb{R P}^{2}$ of degree $m$, in general position with respect to $p \in \mathbb{R} \mathbb{P}^{2}$, and such that $\mathbb{R} A$ satisfies the strong $(m-2)$-condition. Suppose that $\mathbb{R} A$ is a union of embedded pairwise transversal circles. Then there exists a fiberwise model of $\mathbb{R} A$ that can be encoded by a word containing only the symbols $\times_{k}, \in_{k}, \ni_{k}$, and $o_{k}$ (i.e., not containing $\subset_{k}$ and $\supset_{k}$ ).

Proof. Suppose that the encoding word contains $\subset_{k}$ (the case of $\supset_{k}$ is similar); we prove that there exists an admissible isotopy such that the number of " $\supset$ " decreases and the number of " $\subset$ " does not increase. Choosing, if necessary, another line in $\mathcal{L}_{p}$ as the infinite line (this corresponds to a cyclic permutation of the encoding word followed by an evident change of the indices), we may assume that there exists a subword of the form $\subset_{k} \times_{i_{1}} \ldots \times_{i_{n}} u$, where $u$ is either $\supset_{l}$ or $\ni_{l}$. If $n>0$ and $i_{1} \notin\{k-1, k, k+1\}$, then, replacing $\subset_{k} \times_{i_{1}}$ with $\times_{i_{1}} \subset_{k}$ for $i_{1}<k$ or with $\times_{i_{1}-2} \subset_{k}$ for $i_{1}>k$, we can reduce the length of the subword $\times_{i_{1}} \ldots \times_{i_{n}}$ (clearly, such a replacement corresponds to an admissible isotopy). Therefore, we may assume that either $n=0$, or $k-1 \leq i_{1} \leq k+1$.

Case 1: $n=0$ and $u=\supset_{k}$. Replace $\subset_{k} \supset_{k}$ with $o_{k}$.

Case 2: $n=0$ and $u=\supset_{k \pm 1}$. Remove $\subset_{k} \supset_{k \pm 1}$ (zigzag removal).

Case 3: $n=0$ and $u=\ni_{k}$ or $\ni_{k-1}$. Self-intersection (i.e., this contradicts the condition that $\mathbb{R} A$ is a union of embedded circles).

Case 4: $n=0$ and $u=\ni_{k+1}$ (the case of $u=\ni_{k-2}$ is similar). The replacement $\subset_{k} \ni_{k+1} \rightarrow \subset_{k} \times_{k+2} \supset_{k+1} \rightarrow \times_{k} \subset_{k} \supset_{k+1} \rightarrow \times_{k}$.

Case 5: $n=0$, but $u$ is none of the above symbols. Then there exists an isotopy (certainly, nonadmissible!) exchanging $C_{k}$ and $u$. This contradicts the strong $(m-2)$ condition.

Case 6: $n>0, i_{1}=k \pm 1$. Replace $\subset_{k} \times_{k \pm 1}$ with $\in_{k}$ or $\in_{k-1}$.

Case 7: $n>0, i_{1}=k$. Self-intersection.

6.4. Clusters of double points. Let $B$ be a union of embedded circles in $\mathbb{R P}^{2}$, and let $p \in \mathbb{R}^{2} \backslash B$. A subset $D$ of $\mathbb{R P}^{2} \backslash\{p\}$ is called a digon of $B$ with respect to $p$ if

(1) $D$ is homeomorphic to an open disk;

(2) there exists a connected component $B^{\prime}$ of $B$ such that $D$ is a connected component of $\mathbb{R P}^{2} \backslash\left(B^{\prime} \cup\{p\}\right)$;

(3) there are precisely two double points $q_{1}$ and $q_{2}$ of $B$ lying on $\partial D$.

In this case we say that the points $q_{1}$ and $q_{2}$ are connected by the digon $D$. A digon $D$ of $B$ is said to be empty if $D \cap B=\varnothing$.

Let $\Gamma_{B}$ be the graph whose vertices are the double points of $B$ and whose edges correspond to the digons connecting them to each other. We define a cluster of double points of $B$ as the set of vertices of a connected component of $\Gamma_{B}$. A cluster is said to be degenerate if it contains two points connected by two digons having a common side. It is clear that the graph of any nondegenerate cluster $c$ is homeomorphic to a circle, a segment, or a point. In this case we say that a cluster is cyclic, linear or trivial respectively.

The following lemma is evident.

Lemma 6.3. Let $B$ be a union of embedded circles in $R P^{2} \backslash\{p\}$ that are transversal to each other, and let $c$ be a nondegenerate nontrivial cluster of $B$ with respect to $p$. Suppose that the fiberwise arrangement of $B$ is encoded by a word containing only " $\in$ ", " $\ni$ ", " $\times$ ", and "o". Let $\pi_{p}: \mathbb{R P}^{2} \backslash\{p\} \rightarrow \mathbb{R P}^{1}$ be the projection from the point $p$. Then there exist two smooth mappings $\gamma_{1}, \gamma_{2}:[0,1] \rightarrow B$ such that

(1) $\pi_{p} \circ \gamma_{1}=\pi_{p} \circ \gamma_{2}$ (we denote this mapping by $f$ );

(2) $f^{\prime}(t) \neq 0$ for $0<t<1$;

(3) $c=\gamma_{1}([0,1]) \cap \gamma_{2}([0,1])$. 
Lemma 6.4. Let $A$ be a real pseudoholomorphic curve in $\mathbb{R P}^{2}$ of degree $m$ such that $\mathbb{R} A$ is a union of pairwise transversal embedded circles satisfying the strong $(m-2)$-condition with respect to some point $p$. Let $c$ be a nondegenerate cluster of $B$ with respect to $p$, and let $\Gamma$ be a subgraph of $\Gamma_{c}$ homeomorphic to a segment and with edges corresponding to empty digons. Then there exists a fiberwise model of $B$ encoded by a word such that it contains only " $O$ ", " $\times$ ", " $\in$ ", " $\ni$ " and the set of vertices of $\Gamma$ corresponds to a subword of the form $u \times_{k}^{l} v$, where $u \in\left\{\times_{k}, \in_{k}, \in_{k-1}\right\}$ and $v \in\left\{\times_{k}, \ni_{k}, \ni_{k-1}\right\}$.

Moreover, an admissible isotopy transforming $\mathbb{R} A$ into $B$ can be chosen so that it is constant on the complement of an arbitrarily small neighborhood of the union of the lines of $\mathcal{L}_{p}$ intersecting the digons corresponding to the edges of $\Gamma$.

Proof. By Proposition 6.2, there exists a fiberwise model $B$ of $\mathbb{R} A$ encoded by a word in "o", "×", " $\in$ ", " $\ni$ ". Let $\gamma_{1}$ and $\gamma_{2}$ be as in Lemma 6.3. Let $T=\left\{t_{1}, \ldots, t_{n}\right\}$ be a finite subset of $[0,1]$ such that $\gamma_{1}(T)=\gamma_{2}(T)=\left(\right.$ the set of the vertices of $\Gamma$ ) and $t_{1}<\cdots<t_{s}$. We set $N(B)=\operatorname{Card}\left(\left[t_{1}, t_{n}\right] \cap \gamma_{1}^{-1}(S)\right)$, where $S$ is the union of the lines of $\mathcal{L}_{p}$ that pass through the critical points of $B$. Lemma 6.4 can be proved by using induction on $N(B)$, like Proposition 6.2.

Lemma 6.5. Let $A$ be a real pseudoholomorphic curve in $\mathbb{R P}^{2}$ of degree 7. Suppose that $\mathbb{R} A$ is a union of pairwise transversal embedded circles. Let $O$ be an oval of $A$, i.e., a smooth even branch not passing through double points. Suppose that $\mathbb{R} A$ contains no degenerate clusters and satisfies the strong 5-condition with respect to some point $p$ lying inside $O$. Then there exists a fiberwise model of $\mathbb{R} A$ encoded by a word such that it contains only " $o$ ", " $\times$ ", " $\in$ ", " $\ni$ " and each nontrivial cluster of double points $c_{i}$ corresponds to a subword of the form $u_{i} w_{i} v_{i}$, where $u_{i} \in\left\{\times_{k_{i}}, \in_{k_{i}}, \in_{k_{i}-1}\right\}, v_{i} \in$ $\left\{\times_{k_{i}}, \ni_{k_{i}}, \ni_{k_{i}-1}\right\}$ for all $i=1, \ldots, n, w_{i}=\times_{k_{i}}^{l_{i}}$ for $i>1$, and $w_{1}=\times_{k_{1}}^{l_{0}} o_{j_{1}} \ldots o_{j_{s}} \times_{k_{1}}^{l_{1}}$.

Proof. Since there are no degenerate clusters, the curve $\mathbb{R} A$ does not contain any two digons with a common side. Hence, Bézout's theorem for an auxiliary line implies that $\mathbb{R} A$ has at most one nonempty digon. If all digons are empty, our statement follows from Lemma 6.4. Suppose that there is a nonempty digon $D$. We may assume that its vertices belong to $c_{1}$.

Let $\pi_{p}: \mathbb{R P}^{2} \backslash\{p\} \rightarrow \mathbb{R P}^{1}$ be the projection from the point $p$. Lemma 6.4 implies that there exists a fiberwise model $B$ of $\mathbb{R} A$ encoded by a word in " $o$ ", " $\times ", ~ " \in$ ", " $\ni$ " and such that for any empty digon $D^{\prime}$ the open band $\pi_{p}^{-1}\left(\pi_{p}\left(D^{\prime}\right)\right)$ contains no critical points. The end of the proof is the same as for Lemma 6.4, and we omit it also.

6.5. Arrangements of $C_{2} \cup C_{5}$ without nested arcs. It remains to consider the arrangements of $J_{5} \cup C_{2}$ of the series $\mathbf{1}, \mathbf{2}, \mathbf{7}, \mathbf{8 , 2 5}$.

By Theorem 3, we can always choose the center of the pencil of lines inside an oval of the quintic that lies, in its turn, inside the conic.

So, for each of the domains into which $J_{5}$ divides the interior of the conic, we shall check the hypothesis that this domain contains an oval of the quintic (we denote it by $O_{5}$ ). In each case, we shall consider all possible fiberwise arrangements of $C=C_{2} \cup C_{5}$ with respect to $\mathcal{L}_{p}$, where $p$ is chosen inside $O_{5}$.

It is clear that if the center of the pencil of lines is inside $C_{2}$ and inside $O_{5}$, then the arrangement satisfies the strong $(m-2)$-condition (see Subsection 6.3). Therefore, we may consider only the fiberwise models of $C=C_{2} \cup C_{5}$ whose existence is proved in Lemma 6.5. It is easy to check that in this case the fiberwise model of $C_{2} \cup J_{5} \cup O_{5}$ is uniquely determined by the fiberwise arrangement of the clusters of double points on $C_{2}$. Hence, it suffices to try one by one all possible fiberwise arrangements of $n$ points on a conic $C_{2}$, where $n$ is the number of clusters, to construct each time the fiberwise model 
of $C_{2} \cup J_{5} \cup O_{5}$ determined by them, and then, to consider all possible positions for the ovals of the quintic.

We enumerate the intersection points of $C_{2}$ and $J_{5}$ by $0, \ldots, 9$, as in Subsection 0.5. For each choice of $p$, we shall denote the clusters by $c_{1}, c_{2}, \ldots$ clockwise along $C_{2}$ (i.e., in the same order as for the intersection points), starting with the cluster containing the point "0". The domains, i.e., the connected components of $\mathbb{R P}^{2} \backslash\left(J_{5} \cup C_{2}\right)$, will be denoted by $D_{i_{1} i_{2} \ldots}$, where $i_{1}, i_{2}, \ldots$ are the intersection points belonging to the boundary of the domain.

In Table 2, we present the results of the primary analysis of all possible choices of a domain containing $O_{5}$ and of a fiberwise arrangement of the clusters. We do not consider the cases where the choice of a domain containing $O_{5}$ contradicts Bézout's theorem for auxiliary lines. For example, this is the reason why no line of Table 2 corresponds to the choice of $D_{01}$ for the series 7. Indeed, suppose that $p \in D_{01}$ and consider a line passing through $p$ and a point on the arc (67) of the conic. It cuts $O_{5}$ at two points and it cuts also the arcs $(01),(49),(58)$, and (67) of $J_{5}$, so it has too many intersections with $C_{5}$.

Let $p$ be any point in the interior of $C_{2}$, and let $q_{1}, \ldots, q_{n}$ be points on $C_{2}$ in this cyclic order, pairwise noncollinear with $p$. Under these conditions, there exist $2^{n-1}$ fiberwise arrangements of $C_{2}, q_{1}, \ldots, q_{n}$ with respect to $p$. Indeed, choose an affine chart such that $\mathcal{L}_{p}$ is the pencil of vertical lines and $C_{2}$ is the hyperbola $y^{2}-x^{2}=1$. Let $q_{i}=\left(x_{i}, y_{i}\right)$. Let $\left(i_{1}, \ldots, i_{n}\right)$ be the permutation providing $x_{i_{1}}<\cdots<x_{i_{n}}$. We may assume that $i_{1}=1$ and $y_{1}>0$. Then the fiberwise arrangement of $q_{i}$ 's is uniquely determined by the sequence $\left(\operatorname{sgn} y_{i_{2}}, \ldots, \operatorname{sgn} y_{i_{n}}\right)$. In Table 2 , we denote the corresponding fiberwise arrangement of the clusters $c_{1}, \ldots, c_{n}$ by a $(2 \times n)$-matrix whose rows correspond to the branches of the hyperbola. For example, ${ }_{4}^{1}{ }_{4}^{23}$ corresponds to the case where $x_{1}<x_{4}<$ $x_{2}<x_{3}$ and $\operatorname{sgn} y_{1}=\operatorname{sgn} y_{2}=\operatorname{sgn} y_{3}=1=-\operatorname{sgn} y_{4}$.

By Proposition 6.2, it suffices to consider only fiberwise arrangements of $C_{2} \cup J_{5}$ such that all the maxima of the restriction of the $x$-coordinate to $J_{5}$ are situated on $C_{2}$. Such arrangements are uniquely determined by fiberwise arrangements of clusters. We illustrate this in the following example. Consider the first line of Table 2 (The series $\mathbf{2 5}$, $\left.p \in D_{78}\right)$ and the fiberwise arrangement ${ }^{1}{ }_{4}{ }^{23}$ of the clusters. To simplify pictures, we shall present the hyperbola $C_{2}$ as two horizontal lines. We place the points $0, \ldots, 9$ on $C_{2}$ in accordance with the given cluster arrangement (see Figure 30.1) and draw the arcs of $J_{5}$ one by one, starting, for example, with $(01)$. The $x$-coordinate is monotone on each arc. Therefore, there are two choices for the arc (01): Figure 30.2 and Figure 30.3. Since $p \in D_{78}$, this arc must cut the segment contained inside $C_{2}$ and joining $p$ with some point $q$ of the arc (01) of the conic (for our choice of the affine coordinates, this is the vertical ray from $q$ disjoint from the other branch of the hyperbola). For this reason, we must choose Figure 30.2 rather than Figure 30.3. Similarly, we construct all other arcs, obtaining the fiberwise arrangement of $J_{5} \cup C_{2}$ depicted in Figure 30.4. This arrangement contradicts Bézout's theorem for an auxiliary line through the point "4". In such cases, we write "B." in the corresponding square of Table 2.

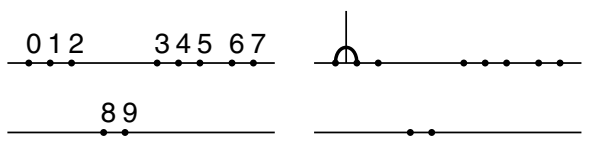

FiguRE 30.1

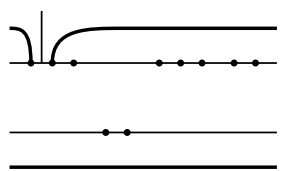

FiguRE 30.2

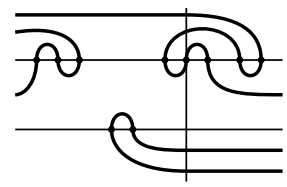

FigURE 30.4 
When drawing a fiberwise arrangement of $J_{5} \cup C_{2}$ by hand, it is convenient to start with the exterior arc of $J_{5}$ passing through infinity and the arcs of $J_{5}$ bounding the domain that contains $O_{5}$. After that, all other arcs will be uniquely determined by the condition that they do not cross the arcs constructed previously.

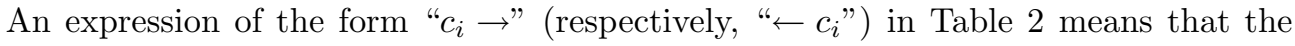
given fiberwise arrangement can be reduced to another arrangement by means of an admissible isotopy (see the definition in Subsection 6.3) such that $c_{i}$ moves to the right (respectively, to the left) and the other clusters are fixed. For example, consider the fiberwise cluster arrangement ${ }^{1}{ }_{3}^{2}$ in the second line of Table 2. It corresponds to the arrangement of $J_{5}, O_{5}$, and $C_{2}$ depicted in Figure 31. By Bézout's theorem for vertical lines, the remaining five ovals of $C_{5}$ must be contained in the band bounded by the vertical lines through the points 9 and 1 . Moreover, they are contained in a smaller band, which we depict in Figure 31 as a gray rectangle. Then there exists an admissible isotopy, shown by arrows in Figure 31, which moves $c_{1}$ (consisting of a single point "0") to the left. As a result, we obtain the fiberwise arrangement $31^{2}$, which can be transformed into ${ }_{23}$ by changing the infinite line.

Finally, we write "O.K." in Table 2 if the corresponding fiberwise arrangement of $C_{2}$, $J_{5}$, and $O_{5}$ should be studied further. Every such arrangement corresponds to a line of Table 3 .

TABLE 2

\begin{tabular}{|c|c|c|c|c|c|c|}
\hline$C_{2} \cup J_{5}$ & $p$ & clusters & \multicolumn{4}{|c|}{ fiberwise arrangements of clusters } \\
\hline & & & \begin{tabular}{|l|l|}
1234 & 123 \\
\end{tabular} & \begin{tabular}{|l|l|}
$12{ }_{4}^{3}$ & 12 \\
34 \\
\end{tabular} & \begin{tabular}{|l|l|}
$1_{4}^{23}$ & $1_{3}^{2}{ }_{4}$ \\
\end{tabular} & \begin{tabular}{|l|l|}
$1_{34}^{2}$ & $1_{234}$ \\
\end{tabular} \\
\hline 25. (M10) & $D_{78}$ & $012,345,67,89$ & \begin{tabular}{|l|l|} 
B. & O.K.
\end{tabular} & \begin{tabular}{|l|l|} 
B. & B.
\end{tabular} & \begin{tabular}{|l|l|} 
B. & B.
\end{tabular} & \begin{tabular}{l|l} 
B. & B.
\end{tabular} \\
\hline & & & 123 & $\begin{array}{l}12 \\
3\end{array}$ & $1_{3}{ }^{2}$ & $\overline{1}_{23}$ \\
\hline 2. (M33) & $\begin{array}{l}D_{01} \\
D_{23} \\
D_{45} \\
D_{78}\end{array}$ & $\begin{array}{l}0,123456,789 \\
012,3456,789 \\
01234,56,789 \\
0123456,7,89\end{array}$ & $\begin{array}{l}\text { B. } \\
\text { B. } \\
\text { B. } \\
\text { B. }\end{array}$ & $\begin{array}{c}\text { B. } \\
\text { B. } \\
\text { B. } \\
\text { O.K. }\end{array}$ & $\begin{array}{c}\leftarrow c_{1} \\
\mathrm{~B} . \\
\mathrm{B} . \\
c_{3} \rightarrow\end{array}$ & $\begin{array}{c}\text { O.K. } \\
\text { O.K. } \\
\text { O.K. } \\
\text { B. }\end{array}$ \\
\hline 7. (M31) & $\begin{array}{c}D_{012349} \\
D_{4589} \\
D_{5678}\end{array}$ & $\begin{array}{l}01234,567,89 \\
01234,567,89 \\
01234,567,89\end{array}$ & $\begin{array}{l}\text { O.K. } \\
c_{3} \rightarrow \\
\text { B. }\end{array}$ & $\begin{array}{c}\text { B. } \\
\leftarrow c_{3} \\
\text { O.K. }\end{array}$ & $\begin{array}{l}\leftarrow c_{3} \\
\text { O.K. } \\
c_{3} \rightarrow\end{array}$ & $\begin{array}{c}\text { B. } \\
\text { O.K. } \\
\text { B. }\end{array}$ \\
\hline 8. (M29) & $\begin{array}{l}D_{01} \\
D_{23}\end{array}$ & $\begin{array}{l}0,1234,56789 \\
012,34,56789\end{array}$ & $\begin{array}{l}\text { B. } \\
\text { B. }\end{array}$ & $\begin{array}{l}\text { B. } \\
\text { B. }\end{array}$ & $\begin{array}{c}c_{2} \rightarrow \\
\mathrm{B} .\end{array}$ & $\begin{array}{l}\text { O.K. } \\
\text { O.K. }\end{array}$ \\
\hline 25. (M10) & $\begin{array}{c}D_{236789} \\
D_{0129}\end{array}$ & $\begin{array}{l}012,345,6789 \\
012,345,6789\end{array}$ & $\begin{array}{l}c_{3} \rightarrow \\
\text { O.K. }\end{array}$ & $\begin{array}{c}\leftarrow c_{3} \\
\mathrm{~B} .\end{array}$ & $\begin{array}{c}\text { O.K. } \\
\text { B. }\end{array}$ & $\begin{array}{l}\text { O.K. } \\
\leftarrow c_{3}\end{array}$ \\
\hline & & & \multicolumn{2}{|c|}{$\overline{12}$} & \multicolumn{2}{|c|}{$1_{2}$} \\
\hline 1. (M30) & $\begin{array}{l}D_{01} \\
D_{23} \\
D_{45} \\
\end{array}$ & $\begin{array}{l}0,123456789 \\
012,3456789 \\
01234,56789\end{array}$ & \multicolumn{2}{|c|}{$\begin{array}{l}c_{2} \rightarrow \\
\text { O.K. } \\
\text { O.K. }\end{array}$} & \multicolumn{2}{|c|}{$\begin{array}{l}\text { O.K. } \\
\text { O.K. } \\
\text { O.K. }\end{array}$} \\
\hline 2. (M33) & $\begin{array}{c}D_{01234569} \\
D_{6789} \\
\end{array}$ & $\begin{array}{l}0123456,789 \\
0123456,789 \\
\end{array}$ & \multicolumn{2}{|c|}{$\begin{array}{l}\text { O.K. } \\
c_{2} \rightarrow\end{array}$} & \multicolumn{2}{|c|}{$\begin{array}{l}c_{1} \rightarrow \\
\text { O.K. }\end{array}$} \\
\hline 8. (M29) & $D_{012349}$ & 01234,56789 & \multicolumn{2}{|c|}{ O.K. } & \multicolumn{2}{|c|}{$c_{1} \rightarrow$} \\
\hline 1. (M30) & $D_{01 \ldots 89}$ & 0123456789 & \multicolumn{4}{|c|}{ O.K. } \\
\hline
\end{tabular}




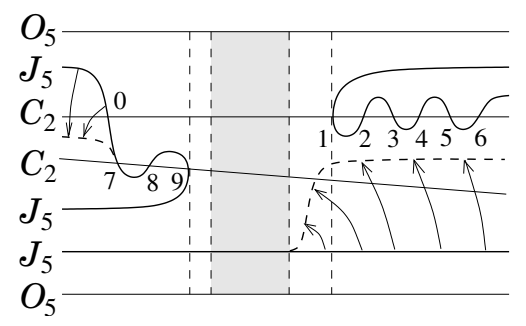

FIGURE 31

TABLE 3

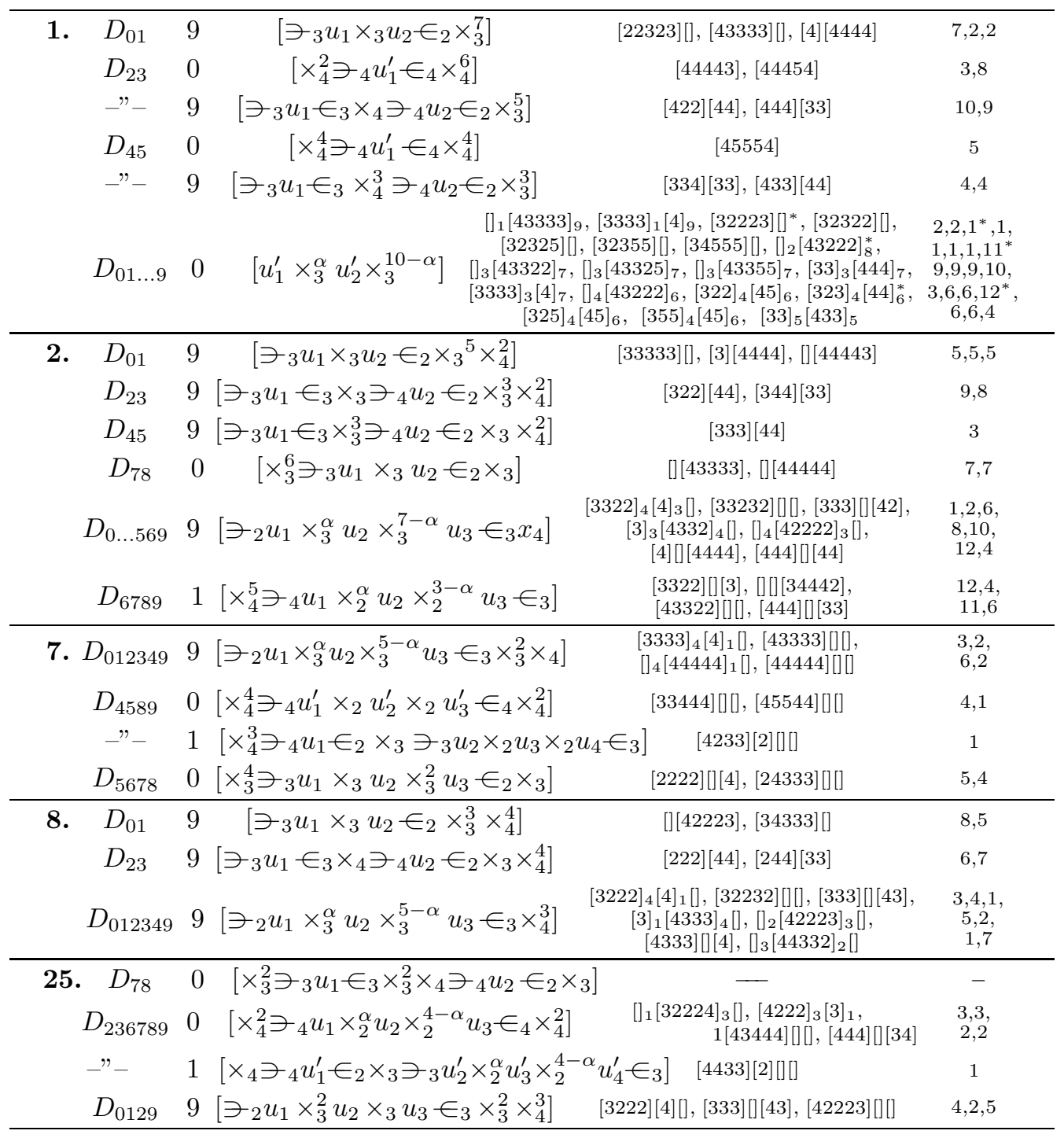


Table 3 is organized like Table 1 above. In the fourth column, we write the encoding words for fiberwise arrangements of $C=C_{5} \cup C_{2}$. The variables $u_{1}, u_{2}, \ldots$ or $u_{1}^{\prime}, u_{2}^{\prime}, \ldots$ (the meaning of the primes is explained below) stand for subwords (including the empty one) of total length 5 and of the form $o_{i_{1}} o_{i_{2}} \ldots$, where $2 \leq i_{1}, i_{2}, \ldots \leq 5$.

The commutation properties of the symbols " $\times$ " and " $O$ " show that if a word contains a subword of the form $u_{i-1}^{(\prime)} \times_{k}^{m} u_{i}^{(\prime)}$, then it suffices to consider only the cases where $u_{i}^{(\prime)}$ either is empty, or starts with $o_{k^{\prime}}$ for $k^{\prime} \neq k+1$. For the same reason, in the case of $\left(1, p \in D_{0 \ldots 9}\right)$, we may assume that either the word $u_{1}^{\prime}$ is empty, or it starts with $o_{3}$, or $u_{1}^{\prime}=o_{2}^{5}$ and $u_{2}^{\prime}=\varnothing$.

The encoding word depends on the choice of the infinite line. Always when it is possible (i.e., in all cases except the last line for the series $\mathbf{1}$ ), we choose it so that it meets $J_{5}$ at three points, and so that the fiberwise arrangement of clusters on the affine chart is as similar as possible to that given in Table 2. In the third column of Table 3, we write the number of the leftmost double point.

Now we explain the difference between $u_{k}$ and $u_{k}^{\prime}$ in the encoding words. Suppose we study the case where the leftmost point has the number $i$ and $O_{5}$ is contained in $D_{\alpha}$. If this domain contains more than one oval, then any of them can be chosen as $O_{5}$. Consider the tangent to $C_{2}$ at the $i$ th point; let us rotate it (in one direction or another) up to the first tangency with an oval of $C_{5}$ contained in $D_{\alpha}$ and choose this oval to be $O_{5}$. The resulting line (denote it by $L$ ) divides the interior of $C_{2}$ into two components, but only one of them can contain other ovals of $D_{\alpha}$. This means that either $o_{2}$ or $o_{5}$ does not occur in the encoding word (depending on the direction of rotation). The absence of the prime of a variable $u_{k}$ means that it may be replaced only by words without $o_{5}$.

In the cases where two different fiberwise models correspond to the same line of Table 2 , we apply this trick only to one of them, to avoid bothering about the compatibility of the choices of $O_{5}$. The fact that this precaution makes sense can be illustrated by the arrangement 1 with $p \in D_{45}$. Analyzing the last column of Table 3 (the meaning of that column will be explained below), we see that, should we consider only words without $o_{5}$, we would have proved the unrealizability of the fifth arrangement of the series $\mathbf{1}$.

Also, this argument cannot be applied to $\left(1 . p \in D_{0 . \ldots 9}\right)$, because in this case it is possible that the line $L$ cuts $J_{5} \cup O_{2}$ at three real points only. Hence, ovals of $C_{5}$ may cross through $L$ during an admissible isotopy that transforms $O_{5}$ into $O_{2}$ and vice versa.

For each fiberwise arrangement listed in Table 3 and satisfying the above restrictions, we checked if the corresponding braid $b$ satisfies the Murasugi-Tristram inequality (see details in [16]), and if the Alexander polynomial $\Delta_{b}(t)$ satisfies the Fox-Milnor condition

$$
\exists f \in \mathbb{Z}[t]: \Delta_{b}(t) \doteq f(t) f\left(t^{-1}\right)
$$

for $e(b)=6$ (i.e., in the case of $\left(\mathbf{1} . p \in D_{0 \ldots 9}\right)$ ), or the condition $\Delta_{b}(t)=0$ for $e(b) \leq 5$ (i.e., in the remaining cases). Here $e(b)$ denotes the exponent sum of $b$, i.e.,

$$
e(b)=\sum k_{j} \quad \text { for } \quad b=\prod \sigma_{i_{j}}^{k_{j}},
$$

and $\doteq$ means equality up to multiplication by a unit of the ring $\mathbb{Z}\left[t, t^{-1}\right]$.

All fiberwise arrangements satisfying these conditions are listed in the fifth column of Table 3 (the data format is the same as in the last column of Table 1). For each of them, in the last column we give the reference to the corresponding (nonfiberwise) arrangement in Subsection 0.5; namely, we write the order number (in accordance with the order in Subsection 0.5) of the corresponding arrangement among all arrangements of the same series. The cases excluded below are marked by an asterisk. The numbers 11 and 12 of the series 1 refer to Figure 32.1 and Figure 32.2. 


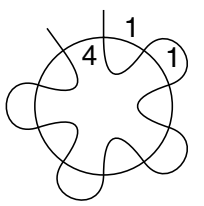

1-11 (unrealizable)

FiguRE 32.1.

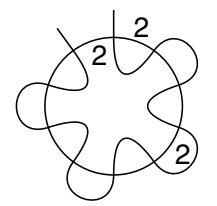

1-12 (unrealizable)

FiguRE 32.2 .

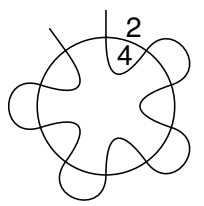

1-7

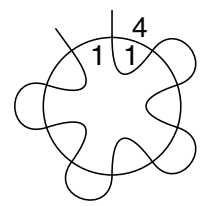

1-2

Figure 33.1. Figure 33.2.

For example, the first line of Table 3 means that we have considered all fiberwise arrangements encoded by

$$
\left[\ni_{3} O_{i_{1}} \ldots o_{i_{k}} \times{ }_{3} o_{i_{k+1}} \ldots o_{i_{5}} \in \in_{2} \times{ }_{3}^{7}\right],
$$

where $0 \leq k \leq 5,2 \leq i_{j} \leq 4$ for all $j$, and if $k<5$, then $i_{k+1}=4$. Among them, those satisfying the Murasugi-Tristram inequality and the condition $\Delta_{b}(t)=0$ are only

$$
\left[\ni_{3} O_{2}^{2} O_{3} O_{2} o_{3} \times{ }_{3} \in \in_{2} \times 7\right], \quad\left[\ni_{3} O_{4} o_{3}^{4} \times{ }_{3} \in_{2} \times{ }_{3}^{7}\right] \quad \text { and }\left[\ni_{3} O_{4} \times{ }_{3} o_{4}^{4} \times{ }_{3} \in_{2} \times{ }_{3}^{7}\right] \text {; }
$$

moreover, the first case corresponds to the 7 th arrangement of the series 1 (i.e., Figure 33.1), and the two other cases both correspond to the second arrangement of the same series (Figure 33.2).

It remains to prove the unrealizability of the arrangements in Figure 32.1 and Figure 32.2. We shall apply the following generalization of the Fox-Milnor theorem.

Theorem 6.6 (Florens [5]). Let $F=F_{1} \cup \cdots \cup F_{\mu}$ be a smooth compact surface embedded into the 4 -ball $\mathbb{B}^{4}$ and such that $L=\partial F \subset \mathbb{S}^{3}=\partial \mathbb{B}^{4}$. Set $L_{i}=\partial F_{i}$. Let $\Delta_{L}\left(t_{1}, \ldots, t_{\mu}\right)$ be the multivariable Alexander polynomial corresponding to the partition $L=L_{1} \cup \cdots \cup L_{\mu}$.

Suppose that the Euler characteristic $\chi(F)$ is equal to one. Then there exists a polynomial $f\left(t_{1}, \ldots, t_{\mu}\right)$ with integral coefficients such that

$$
\Delta_{L}\left(t_{1}, \ldots, t_{\mu}\right) \prod_{i=1}^{\mu}\left(t_{i}-1\right)^{-\chi\left(F_{i}\right)} \doteq f\left(t_{1}, \ldots, t_{\mu}\right) f\left(t_{1}^{-1}, \ldots, t_{\mu}^{-1}\right),
$$

where $\doteq$ means equality up to multiplication by units of the ring $\mathbb{Z}\left[t_{1}^{ \pm 1}, \ldots, t_{\mu}^{ \pm 1}\right]$.

Let $b$ be the braid associated with one of the fiberwise arrangements listed in the last line of the series $\mathbf{1}$ in Table 3. As was explained in [13], if this fiberwise arrangement is a fiberwise model of a real pseudoholomorphic curve $C=C_{2} \cup C_{5}$, then the closure $L$ of $b$ is isotopic to the boundary of a surface $F$ that is the intersection of the complexification of $C$ with some 4-ball embedded into $\mathbb{C P}^{2}$. Let $L=L_{2} \cup L_{5}$ and $F=F_{2} \cup F_{5}$ be the partitions corresponding to the partition $C=C_{2} \cup C_{5}$. By the Riemann-Hurwitz formula, we have $\chi(F)=\operatorname{deg} C-e(b)=7-6=1$. Hence, the multivariable Alexander polynomial $\Delta_{L}\left(t_{2}, t_{5}\right)$ associated with the partition $L=L_{2} \cup L_{5}$ must be of the form (12). An explicit computation shows that this is not so in the cases marked by an asterisk in Table 3.

Remark 6.7. The arrangement in Figure 32.1 (respectively, in Figure 32.2) can also be excluded without using Theorem 6.6. It can be checked that if the center of the pencil of lines is chosen inside an oval contained in the digon (12) (respectively, (34)), then all fiberwise models contradict the Murasugi-Tristram inequality. As above, it suffices to consider only models corresponding to all possible fiberwise arrangements of the clusters $c_{1}=(01)$ and $c_{2}=(2 \ldots 9)$ (respectively, $c_{1}=(0123)$ and $\left.c_{2}=(456789)\right)$ on the conic $C_{2}$, which can be represented in this case by an ellipse in the affine plane. By Proposition 6.2 , it suffices to consider only the cases where the extrema of the $x$-coordinate on $C_{2}$ are the leftmost or rightmost points of the clusters. 
Remark 6.8. The arrangements in Figures 32.1-32.2 are pseudoholomorphically unrealizable, but the corresponding Alexander polynomials in one variable do not contradict the Fox-Milnor theorem. Perhaps, this is merely a coincidence. But its probability is rather small (contrary to a situation where, say, the Murasugi-Tristram inequality is fulfilled occasionally). So, the following question arises naturally: is this an indication that these arrangements are realizable as the sets of real points of some objects (for instance, flexible curves in the sense of Viro) that generalize the real algebraic curves but in a weaker sense than the real pseudoholomorphic curves?

\section{§7. Completion of the Classification}

7.1. Restrictions for $C_{5} \cup L_{1} \cup L_{2}$ deduced from the restrictions for $C_{5} \cup C_{2}$ proved before. The Bézout theorem for auxiliary lines shows that if an $M$-quintic $C_{5}$ and two lines $L_{1}, L_{2}$ realize an arrangement of the type $(1,1)$ (respectively, $(1,3)$ ), then the odd branch $J_{5}$ of $C_{5}$ is arranged with respect to $L_{1} \cup L_{2}$ in one of the 8 (respectively, 19) ways corresponding to the series in Subsection 0.6 (respectively, in Subsection 0.7). The question is how the ovals of the quintic are distributed between the components of the complement to $J_{5} \cup L_{1} \cup L_{2}$.

For most of the series, in particular, for all the series of the type $(1,1)$, the answer to this question follows from the partial classification of the arrangements of $C_{5} \cup C_{2}$ with one passage through infinity, which was already obtained in $\S 6$, combined with the classification of the arrangements of $C_{5} \cup C_{2}$ with five passages through infinity or with five nested interior arcs, which evidently reduces to Polotovski1's classification of arrangements of an $M$-quintic and a line.

Example 7.1. The series 8 of the type $(1,1)$, i.e., the series $E$ according to 9 . We denote the components of the complement to $J_{5} \cup L_{1} \cup L_{2}$ as in Figure 34.1 (as usual, each of the letters $a, b, \ldots$ denotes simultaneously the domain and the number of ovals of the quintic contained in it). Bézout's theorem for auxiliary lines implies that there are no ovals in other domains. We perturb $L_{1} \cup L_{2}$ into a nonsingular conic as shown in Figure 34.2. We obtain the arrangement of $J_{5} \cup C_{2}$ of the series $\mathbf{2 8}$. The classification for this series is not completed yet (on the contrary, in $\S 5$ we decided to reduce this case to the classification of $C_{5} \cup L_{1} \cup L_{2}$ ). Let us try then to redraw this arrangement as shown in Figure 34.3 and to perturb it as in Figure 34.4. This time, we obtain an arrangement of $J_{5} \cup C_{2}$ of the series 24, whose classification was completed in Subsection 6.2. This classification shows that only two cases are possible: (1) $a=d=0, b=4, c=2$ or (2) $c=0, a=b=d=2$. Both of them can be found in the list of realized arrangements in Subsection 0.6.

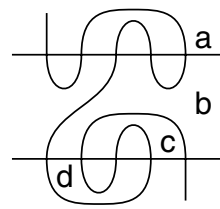

FigURE 34.1

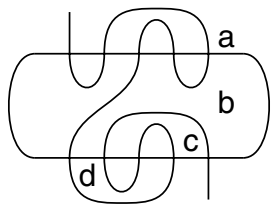

FigURE 34.2

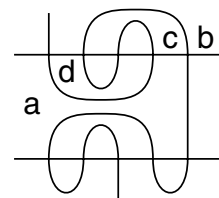

FigURE 34.3

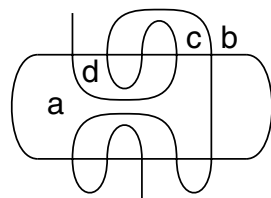

FIgURE 34.4

Example 7.2. The series 16 of the type $(1,3)$. We denote the domains as in Figure 35.1. Since one of two possible perturbations of the two lines into a conic provides an arrangement with three passages through infinity, whose classification we did not even start yet (it will be obtained in Subsection 7.3), we shall consider only the perturbation depicted in Figure 35.2. This is the series $\mathbf{1 3}$ where, in accordance with Subsection 6.2, only one arrangement is realizable, and we have $a+b=c=d=2$ for it. It remains to show that $a=0$ and $b=2$. This follows from the fact that, after forgetting one of the lines, only 
these values of $a$ and $b$ provide an arrangement that does not contradict the Polotovskil classification of arrangements of a quintic and a line.

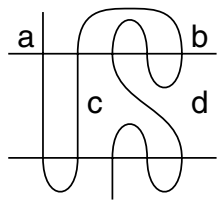

FigURE 35.1

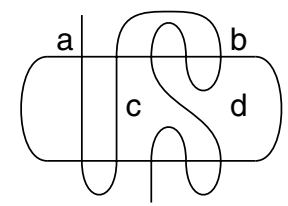

FIGURE 35.2

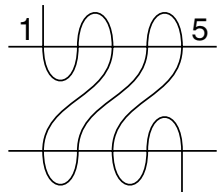

FigURE 36.1

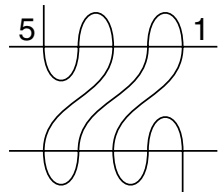

FiguRE 36.2

Proceeding as in these examples, it is not difficult to exclude all arrangements absent in Subsection 0.6-0.7 except for the series 1, 2, 3, 9, 10, 12, 14 of the type $(1,3)$ and except for two more arrangements of the series $\mathbf{5}$ of the type $(1,3)$, which are depicted in Figures 36.1, 36.2.

7.2. End of proof of Theorem 2. In all the remaining cases, we apply the MurasugiTristram inequality. For the arrangements in Figures 36.1-36.2, the choice of the center of the pencil of lines in the solitary oval provides unique fiberwise models $\left[\ni_{4} O_{3}^{5} \times_{3} \in_{4} \times{ }_{4}^{2}\right.$ $\left.\times_{3}^{2} \times_{4}^{2} \times{ }_{3}^{2}\right]$ and $\left[\supset_{4} o_{4}^{4} \times_{2} \times{ }_{3}^{3} \times{ }_{2}^{2} \times{ }_{3}^{2} \times 2{ }_{2}^{3} \subset_{3}\right]$, respectively.

In the other cases, we choose the center of the pencil of lines on one of the lines $L_{1}, L_{2}$ (let it be $L_{1}$ ) inside the nest formed by arcs of the quintic. We choose $L_{1}$ as the infinite line. The results of the computations are presented in Table 4, which is organized like Tables 1 and 3 in Subsections 6.2 and 6.4. As in Subsection 6.2, the variables $u_{i}$ stand only for the subwords of the form $o_{i_{1}} o_{i_{2}} \ldots$ that provide realizable fiberwise arrangements of $C_{5}$ after dropping $L_{2}$. If $u_{i}$ follows $\times_{k}$, then either it is empty, or it starts with $o_{k+1}$.

TABLE 4

\begin{tabular}{cccc}
\hline $\mathbf{1 .}$ & {$\left[\supset_{2} u_{1} \in_{2} \times_{2} \ni_{1} u_{2} \in_{1} \times_{2}\right]$} & {$[222][322],[1][22222]$} & 1,2 \\
$\mathbf{2 .}$ & {$\left[\times_{3} \ni_{2} u_{1} \times_{1}^{\alpha} u_{2} \times_{1}^{3-\alpha} u_{3} \subset_{1}\right]$} & {$[322244]\left[[],[323311][],[]_{1}[211111]_{2}[]\right.$} & $3,1,2$ \\
$\mathbf{3 .}$ & {$\left[\supset_{1} u_{1} \times_{1} u_{2} \times_{1} u_{3} \in_{2} \times_{3}^{2}\right]$} & {$[44][[2223],[1133][[23],[11111][2][]$} & $3,1,2$ \\
$\mathbf{9 .}$ & {$\left[\supset_{1} u_{1} \ni_{2} \times_{2} \times_{1}^{2} \times_{2}\right]$} & {$[112222],[111111]$} & 2,1 \\
$\mathbf{1 0 .}$ & {$\left[\supset_{2} u_{1} \times_{1} u_{2} \in_{1} \times_{2} \times_{3}^{2}\right]$} & $[444444]][,[333344][],[3333][22],[][222222]$ & $1,2,2,1$ \\
$\mathbf{1 2 .}$ & {$\left[\supset_{1} u_{1} \in_{2} \times_{2} \ni_{1} u_{2} \times_{1} u_{3} \in_{3}\right]$} & {$[1111][22],[1122][33]$} & 1,2 \\
$\mathbf{1 4 .}$ & {$\left[\supset_{1} u_{1} \times_{1} u_{2} \in_{1} \times_{2} \ni_{2} u_{3} \in_{2}\right]$} & {$[113][[223]$} & 1 \\
\hline
\end{tabular}

7.3. End of proof of Theorem 1. By Corollary 5.2, the classification of the series not considered in $\S 6$ follows from Theorem 2 .

Appendix A. A New proof of the algebraic unRealizability of a Certain PSEUDOHOLOMORPHIC $M$-CURVE OF BIDEGREE $(4,8)$ ON THE QUADRATIC CONE

Proposition A.1. There is no real algebraic curve of bidegree $(4,8)$ on the Hirzebruch surface $\mathbb{R} \mathcal{F}_{2}$ (i.e., on the blown-up quadratic cone) that is arranged with respect to the exceptional divisor $E$ and one of the fibers $F$ as depicted in Figure 37, where $\mathbb{R} \mathcal{F}_{2}$ is represented by a rectangle with opposite sides identified. The horizontal sides represent $E$ and the vertical sides represent $F$.

This result was proved in 21] with the help of the Hilbert-Rohn method. Note that Figure 37 is realizable by a real pseudoholomorphic curve in an almost complex structure where the self-intersection number of $E$ is equal to -2. In this Appendix, I give a new proof of Proposition A.1, similar to the proof of the algebraic unrealizability of Figure 16.12, given in Subsection 4.4, but here I use also Agnihotri-Woodward inequalities. 


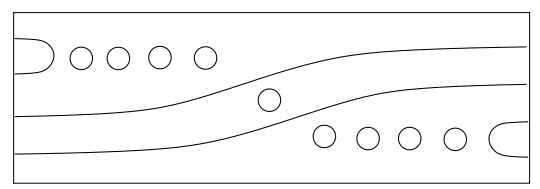

FiguRE 37

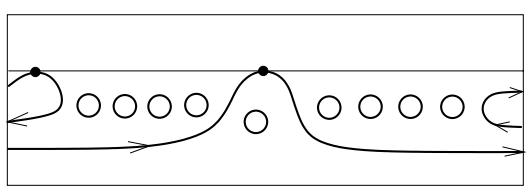

Figure 38

In my opinion, the new proof is simpler and "more reliable" than the old one (unfortunately, proofs based on the Hilbert-Rohn method sometimes have mistakes because some cases of possible degeneration are missed; the first version of $\S 4$ of this text nearly contributed to the list of such erroneous proofs).

On the other hand, the result stated in Proposition A.1 was obtained in [21] as a corollary to a stronger result stating that the $(M-1)$-curve obtained from Figure 37 by removing the central oval is algebraically unrealizable. At the present time, I do not know any proof of this stronger result other than the proof given in 21] and based on the Hilbert-Rohn method.

In this context, it is natural to ask the following question. Is the arrangement of an $(M-1)$-quintic and two lines on $\mathbb{R P}^{2}$ obtained from Figure 16.12 by deleting the solitary empty oval (i.e., by erasing the digit "1" on the picture) algebraically realizable?

Proof of Proposition A.1. If Figure 37 is algebraically realizable, then the cubic resolvent $R$ should be arranged with respect to the core $L$ as in Figure 38 (see Remark 4.5). The braid associated with the curve $A=R \cup L$ has the form

$$
b=\sigma_{3}^{-2} \sigma_{2}^{-5} \sigma_{1}^{-1} \sigma_{2} \sigma_{1}^{-1} \sigma_{3}^{-2} \sigma_{2}^{-1} \sigma_{1} \sigma_{2}^{-4} \Delta^{4} .
$$

The curve $R$ is maximal, and its complex orientation must be as shown in Figure 38, because otherwise the linking number of positive and negative strings would not be equal to zero. Let $H, N, N_{R}$, and $N_{0}$ denote the same as in $\S 4$.

Computing the Euler characteristics and linking numbers (as in $\S 4$ ), we see that $N$ consists of two connected components $N_{1}, N_{2}$ that cover $H$ once and twice, respectively. The projection $N_{2} \rightarrow H$ has two branching points, and each of $N_{1}$ and $N_{2}$ touches $N_{0}$ once.

Hence, coloring the strings of $b$ into the colors $\left(t_{2}, t_{1}, t_{2}, t_{0}\right)$, we conclude that it must have the form $b=b_{1}^{(1)} b_{1}^{(2)} b_{4}^{(1)} b_{4}^{(2)}$, where the colored braid $b_{1}^{(j)}, j=1,2$, is a conjugate of $\sigma_{1}:\left(t_{2}, t_{2}, t_{0}, t_{1}\right) \rightarrow\left(t_{2}, t_{2}, t_{0}, t_{1}\right)$, and the colored braids $b_{4}^{(j)}, j=1,2$, are conjugates of $\sigma_{1}^{4}:\left(t_{0}, t_{j}, t_{3-j}, t_{2}\right) \rightarrow\left(t_{0}, t_{j}, t_{3-j}, t_{2}\right)$.

The images of $b_{1}^{(j)}$ and $b_{4}^{(j)}$ under the Burau representation have the spectra $\left(-t_{2}, 1,1\right)$ and $\left(t_{0}^{2} t_{j}^{2}, 1,1\right)$, respectively.

A computation similar to that in [15] leads to a contradiction with one of the AgnihotriWoodward inequalities for the values of the parameters $t_{0}=t_{2}=i=\sqrt{-1}, t_{1}=-\bar{\tau}=$ $(1+i \sqrt{3}) / 2$.

\section{Appendix B. Example of an unRemovable Zigzag on a ReAl Algebraic CuRve}

Proposition B.1. a) There exists a nonsingular real algebraic curve of bidegree $(3,6)$ on $\mathcal{F}_{2}$ (see Definition 2.10) whose fiberwise arrangement is encoded by $w=\left[\supset_{1} o_{1} \subset_{1} \supset_{2}\right.$ $\left.o_{2} \subset_{2} \supset_{1} \subset_{2} \supset_{1} \subset_{2}\right]$.

b) There is no nonsingular real algebraic curve of bidegree $(3,6)$ on $\mathcal{F}_{2}$ whose fiberwise arrangement is encoded by $w^{\prime}=\left[\supset_{1} o_{1} o_{2} \subset_{2} \supset_{1} \subset_{2} \supset_{1} \subset_{2}\right]$.

Proof. a) Such a curve can easily be constructed with the help of Viro's method, by subdividing the triangle $(0,0)-(6,0)-(0,3)$ by the segment $(3,0)-(0,3)$. The gluing 
of charts corresponds to the representation of $w$ in the form $w=u_{1} v u_{2}$, where $u=\supset_{1}$ $o_{1} \subset_{1}, v=\supset_{2} o_{2} \subset_{2} \supset_{1} \subset_{2}$, and $u_{2}=\supset_{1} \subset_{2}$. Here $v$ represents the chart in the triangle $(0,0)-(3,0)-(0,3)$, and $u_{1} u_{2}$ represents the chart in $(3,0)-(6,0)-(0,3)$.

b) This follows from [18, Lemma 6.6], which states that the encoding word of a curve of bidegree $(3,3 n)$ on $\mathcal{F}_{n}$ cannot contain more than $n$ subwords of the form $\supset_{1} \subset_{2}$ or $\supset_{2} \subset_{1}$.

The word $w^{\prime}$ is obtained from $w$ by deleting the subword $\subset_{1} \supset_{2}$, i.e., by a zigzag removal.

Remark. A generalization of [18, Lemma 6.6] (which was used in the proof of part (b) of Proposition B.1) for any degree was recently obtained by Brugallé [1.

\section{Appendix C. Smooth pseudoholomorphic curves of Bidegree $(4,16)$ on $\mathcal{F}_{4}$} WHOSE (NONFIBERWISE!) ISOTOPIC TYPE IS UNREALIZABLE ALGEBRAICALLY

We remind the reader that $\mathcal{F}_{n}$ stands for the $n$th Hirzebruch surface (see Definition 2.10, where the choice of a real structure on $\mathcal{F}_{n}$ was also described). When talking of real pseudoholomorphic curves on $\mathcal{F}_{n}$, we consider only tame conj-invariant almost complex structures that admit a pseudoholomorphic curve $E$ whose self-intersection index is equal to $-n$ (an exceptional curve). Such a curve is necessarily real.

For general conj-invariant structures on $\mathcal{F}_{n}$ with $n \geq 2$, in 24 some real pseudoholomorphic curves $A$ were constructed in such a way that the isotopy type of the curve $\mathbb{R} A$ is unrealizable algebraically. The reason for the algebraic unrealizability of the examples in 24 is that otherwise the number of real points of the intersection of $\mathbb{R} A$ and $\mathbb{R} E$ would have been greater than the intersection index A.E. In particular, this implies that the examples in 24] are not realizable in almost complex structures with an exceptional curve.

If an exceptional curve exists, then restrictions based on intersection indices with auxiliary rational curves do not apply; however, the Hilbert-Rohn method and the techniques of cubic resolvents are applicable. These methods were employed in [21, 22] (see also Appendix A above) to prove that some fiberwise isotopy types realizable pseudoholomorphically are algebraically unrealizable. However, the curves in these examples are isotopic (not fiberwise) to algebraic curves.

In this Appendix, by the method of cubic resolvents, it is proved that some (nonfiberwise) isotopy types of curves of bidegree $(4,16)$ on $\mathcal{F}_{4}$ are algebraically unrealizable. Moreover, these isotopic types are realizable by real pseudoholomorphic curves relative to a tame complex structure possessing an exceptional curve. I obtained this result at the time when the results of [16] were proved; I did not include it in [16] because I was going to write a separate paper about applications of the method of cubic resolvents. But this method has already been presented in [18, 22] and the present article, so there is no need for such a separate paper any longer, and I have decided to write this Appendix.

A circle smoothly embedded in $\mathbb{R} \mathcal{F}_{n}$ is called an oval if it bounds a disk (since $\mathbb{R} \mathcal{F}_{n}$ is the torus or the Klein bottle, this condition is equivalent to that of being null homologous). The disk bounded by an oval is called the interior of the oval. If a curve has only ovals, we shall use Viro's code to identify its isotopy type (when doing so, we assume that all embedded circles are ovals).

Proposition C.1. The isotopy types $1 \sqcup 1\langle 1\rangle \sqcup 1\langle 18\rangle$ and $1 \sqcup 1\langle 7\rangle \sqcup 1\langle 12\rangle$

a) are unrealizable by real algebraic curves of bidegree $\langle 4,16\rangle$ on $\mathbb{R} \mathcal{F}_{4}$;

b) are realizable by J-holomorphic curves of bidegree $\langle 4,16\rangle$ on $\mathbb{R} \mathcal{F}_{4}$ for some tame conj-invariant almost complex structure $J$ with an exceptional $J$-holomorphic curve. 
Proof. a) If one of these isotopy types is realizable by a real pseudoholomorphic curve $A$, then, up to zigzags and symmetry removal, the fiberwise isotopy type of $\mathbb{R} A$ is either

or

$$
\begin{array}{r}
A\left(\alpha_{1}, \alpha_{2}, \alpha_{3}\right)=\left[\supset_{1} \subset_{1} o_{2}^{\alpha_{1}} o_{3} o_{2}^{\alpha_{2}} \supset_{1} \subset_{2} o_{2}^{\alpha_{3}}\right], \\
\alpha_{1}+\alpha_{2}+\alpha_{3}=19, \\
\alpha_{3} \in\{1,7,12,18\}, \quad \alpha_{1} \geq \alpha_{2},
\end{array}
$$

$$
B\left(\alpha_{1}, \alpha_{2}\right)=\left[\supset_{1} \subset_{1} o_{2}^{\alpha_{1}} \supset_{1} o_{1} \subset_{1} o_{2}^{\alpha_{2}}\right], \quad \alpha_{1}+\alpha_{2}=19, \quad \alpha_{3} \in\{1,7\} .
$$

Passing to cubic resolvents, we obtain an $M$-curve of bidegree $(3,24)$ on $\mathcal{F}_{\infty}$ of the fiberwise isotopy type $A^{\prime}\left(\alpha_{1}, \alpha_{2}, \alpha_{3}\right)=\left[o_{2} o_{1}^{\alpha_{1}} o_{2} o_{1}^{\alpha_{2}} o_{2} o_{1}^{\alpha_{3}}\right]$ or $B^{\prime}\left(\alpha_{1}, \alpha_{2}\right)=\left[o_{2} o_{1}^{\alpha_{1}} o_{2}^{2} o_{1}^{\alpha_{2}}\right]$, respectively.

The algebraic length of the corresponding braids is 2. From the quasipositivity criterion (see [19]) for braids of 3 strings it easily follows that the isotopy type $A^{\prime}$ is pseudoholomorphically realizable only in the case where $\left(\alpha_{1}, \alpha_{2}, \alpha_{3}\right) \in\{(17,1,1),(11,7,1)$, $(11,1,7),(7,5,7)\}$, and the isotopy type $B^{\prime}$ is unrealizable for any values of $\left(\alpha_{1}, \alpha_{2}\right)$.

We denote by $b=b\left(\alpha_{1}, \alpha_{2}, \alpha_{3} ; e_{1}, e_{2}\right)$ the braid corresponding to the fiberwise isotopy type $A\left(\alpha_{1}, \alpha_{2}, \alpha_{3}\right)$. It depends on two integer-valued parameters $e_{1}, e_{2}$ and has the form

$$
b=a \sigma_{3}^{1+e_{1}} \sigma_{1}^{1-e_{1}} \sigma_{2}^{-\alpha_{1}} \tau_{2,3} \sigma_{3}^{-1} \tau_{3,2} \sigma_{2}^{-\alpha_{2}} a \sigma_{3}^{1+e_{2}} \sigma_{1}^{1-e_{2}} \sigma_{2}^{-\alpha_{3}} \Delta^{4},
$$

where

and

$$
a=\sigma_{1}^{-1} \sigma_{3}^{-1} \sigma_{2}^{-1}, \quad \tau_{2,3}=\tau_{3,2}^{-1}=\sigma_{3}^{-1} \sigma_{2}
$$

$$
\Delta=\sigma_{1} \sigma_{2} \sigma_{3} \sigma_{1} \sigma_{2} \sigma_{1} .
$$

If $e_{1} \not \equiv e_{2} \bmod 2$, then the image of $b$ in the permutation group $S_{4}$ is $(13)(23)$; i.e., the closure of $L$ has two components. Their linking number is -3 , which is impossible. So, we assume that $e_{1} \equiv e_{2} \bmod 2$. In this case the image of the braid in $S_{4}$ is trivial. The calculation of Euler characteristics implies that $L$ bounds a three-component surface in the ball $B^{4}$. The calculation of the linking numbers for components shows that two cases are possible:

(i) $e_{1} \equiv e_{2} \equiv 0 \bmod 2$ and $e_{2}-e_{1}=10$;

(ii) $e_{1} \equiv e_{2} \equiv 1 \bmod 2$ and $e_{2}-e_{1}=11-\alpha_{3}$.

By [22, Lemma 3.7] we see that $\left(2\left|e_{1}\right|-1\right)+\left(2\left|e_{2}\right|-1\right) \leq 12$, i.e., $\left|e_{1}\right|+\left|e_{2}\right| \leq 7$ (see the end of Subsection 4.1). This eliminates case (i) for all $\alpha_{3} \in\{1,7\}$, and also case (ii) for $\alpha_{3}=1$.

It remains to analyze case (ii) for $\alpha_{3}=7$. The Murasugi-Tristram inequality shows that the determinant $d$ of $L$ must be 0 . Expressing $e_{2}$ in terms of $e_{1}$ and calculating $d$ with the help of the program ssmW in [16, Appendix] (or directly, with the help of Höritz matrices, as in [21]), we obtain $d=24\left(347-876 e_{1}-223 e_{1}^{2}\right)$ for $\alpha_{1}=7$ and $d=24\left(31 e_{1}+137\right)\left(3-e_{1}\right)$ for $\alpha_{1}=11$. In the first case, the equation $d\left(e_{1}\right)=0$ has no integral solutions, and in the second case there is one solution $e_{1}=3$, yielding $e_{2}=e_{1}+11-\alpha_{3}=3+11-7=7$, which contradicts the inequality $\left|e_{1}\right|+\left|e_{2}\right| \leq 7$ (this solution corresponds to the pseudoholomorphic curve constructed in [16, §5.2]).

b) The isotopy type $1 \sqcup 1\langle 7\rangle \sqcup 1\langle 12\rangle$ was realized in [16, §5.2].

In order to realize $1 \sqcup 1\langle 1\rangle \sqcup 1\langle 18\rangle$, we observe that the braid $b\left(17,1,1 ; e_{1}, 10-e_{1}\right)$ treated above does not depend on $e_{1}$ and is quasipositive. Moreover, for $e_{2}=10-e_{1}$, the braid

$$
a \sigma_{3}^{1+e_{1}} \sigma_{1}^{1-e_{1}} \sigma_{2}^{-1} \sigma_{1}^{-1} \sigma_{2}^{-16} \tau_{2,3} \sigma_{3}^{-1} \tau_{3,2} \sigma_{2}^{-1} a \sigma_{3}^{1+e_{2}} \sigma_{1}^{1-e_{2}} \sigma_{2}^{-1} \Delta^{4}
$$

is also quasipositive; this braid corresponds to the fiberwise arrangement $\left[\supset_{1} \subset_{1} O_{2} \supset_{2}\right.$ $\left.\times_{1} \supset_{2} o_{2}^{15} O_{3} O_{2} \supset_{1} \subset_{2} O_{2}\right]$. Since the algebraic length of this braid is 1 , to check quasipositivity it suffices to show that this braid is conjugate to a standard generator. 


\section{REFERENCES}

[1] E. Brugallé, A bound on the number of jumps of real algebraic curves in $\Sigma_{n}$, Preprint, 2006.

[2] A. I. Degtyarev and V. M. Kharlamov, Topological properties of real algebraic varieties: Rokhlin's way, Uspekhi Mat. Nauk 55 (2000), no. 4, 129-212; English transl., Russian Math. Surveys 55 (2000), no. 4, 735-814. MR.1786731 (2001h:14077)

[3] T. Fiedler, Congruence mod 16 for symmetric M-curves (copy of a handwritten paper).

[4] S. Fiedler-LeTouzé and S. Yu. Orevkov, A flexible affine $M$-sextic which is algebraically unrealizable, J. Algebraic Geom. 11 (2002), 293-310. MR1874116 (2002h:14095)

[5] V. Florens, On the Fox-Milnor theorem for the Alexander polynomial of links, Int. Math. Res. Not. 2004, no. 2, 55-67. MR 2040323 (2005b:57014)

[6] M. A. Gushchin, Construction of some arrangements of conics and $M$-quintics with a point at infinity, Vestnik Nizhegorod. Gos. Univ. Mat. Vyp. 1(2) (2004), 43-52. (Russian)

[7] $\longrightarrow$ A conic and $M$-quintic with a point at infinity, Zap. Nauchn. Sem. S.-Peterburg. Otdel. Mat. Inst. Steklov. (POMI) 329 (2005), 14-27; English transl., J. Math. Sci. (N.Y.) 140 (2007), no. 4, 502-510. MR2215328 (2007a:14063)

[8] V. M. Kharlamov and O. Ya. Viro, Extensions of the Gudkov-Rohlin congruence, Topology and Geometry—Rohlin Seminar, Lecture Notes in Math., vol. 1346, Springer, Berlin, 1988, pp. 357-406. MR0970085 (90a:14030)

[9] A. B. Korchagin and G. M. Polotovskii, On arrangements of a plane real quintic curve with respect to a pair of lines, Commun. Contemp. Math. 5 (2003), no. 1, 1-24. MR1958018 (2004a:14060)

[10] _ On arrangements of a plane real quintic curve with respect to a pair of lines. II (in preparation).

[11] A. Korchagin and E. I. Shustin, Sixth-degree affine curves and smoothings of a nondegenerate sixthorder singular point, Izv. Akad. Nauk SSSR Ser. Mat. 52 (1988), no. 6, 1181-1199; English transl., Math. USSR-Izv. 33 (1989), no. 3, 501-520. MR0984215 (90h:14043)

[12] S. M. Natanzon, Automorphisms of the Riemann surface of an $M$-curve, Funktsional. Anal. i Prilozhen. 12 (1978), no. 3, 82-83; English transl., Funct. Anal. Appl. 12 (1978), no. 3, 228-229 (1979). MR0509395 (82b:14020)

[13] S. Yu. Orevkov, Link theory and oval arrangements of real algebraic curves, Topology 38 (1999), 779-810. MR 1679799 (2000b:14066)

[14] _ Projective conics and $M$-quintics in general position with a maximally intersecting pair of ovals, Mat. Zametki 65 (1999), no. 4, 632-636; English transl., Math. Notes 65 (1999), no. 3-4, 528-532. MR.1715066 (2000h:14050)

[15] _ Quasipositivity test via unitary representations of braid groups and its applications to real algebraic curves, J. Knot Theory Ramifications 10 (2001), 1005-1023. MR.1867106 (2002m:20060)

[16] Classification of flexible M-curves of degree 8 up to isotopy, Geom. Funct. Anal. 12 (2002), 723-755. MR1935547 (2003h:14090)

[17] _ Riemann existence theorem and construction of real algebraic curves, Ann. Fac. Sci. Toulouse Math. (6) 12 (2003), 517-531. MR2060598 (2005d:14084)

[18] Construction of arrangements of $M$-quartics and $M$-cubics with the maximal intersecting of an oval and the odd branch, Vestnik Nizhegorod. Gos. Univ. Mat. Modelirovanie Optim. Upravlenie Vyp. 1(25) (2002), 12-48. (Russian)

[19] —, Quasipositivity problem for 3-braids, Turkish J. Math. 28 (2004), 89-93. MR2056762 (2005d:20063)

[20] S. Yu. Orevkov and G. M. Polotovskiĭ, Projective $M$-cubics and $M$-quartics in general position with a maximally intersecting pair of ovals, Algebra i Analiz 11 (1999), no. 5, 166-184; English transl., St. Petersburg Math. J. 11 (2000), no. 5, 837-852. MR.1734352 (2000m:14067)

[21] S. Yu. Orevkov and E. I. Shustin, Flexible algebraically unrealizable curves: Rehabilitation of Hilbert-Rohn-Gudkov approach, J. Reine Angew. Math. 551 (2002), 145-172. MR1932177 (2005b:14100)

[22] , Pseudoholomorphic algebraically unrealizable curves, Mosc. Math. J. 3 (2003), 1053-1083. MR 2078573 (2005e:14081)

[23] G. M. Polotovskiı̌, A catalogue of $M$-decomposable curves of order six, Dokl. Akad. Nauk SSSR 236 (1977), no. 3, 548-551; English transl. in Soviet Math. Dokl. 18 (1977). MR0460339 (57:333) 
[24] J.-Y. Welschinger, Courbes algébriques réelles et courbes flexibles sur les surfaces réglées de base $\mathbb{C P}^{1}$, Proc. London Math. Soc. (3) 85 (2002), 367-392. MR1912055 (2003f:14064)

[25] V. I. Zvonilov, Complex topological invariants of real algebraic curves on a hyperboloid and an ellipsoid, Algebra i Analiz 3 (1991), no. 5, 88-108; English transl., St. Petersburg Math. J. 3 (1992), no. 5, 1023-1042. MR1186237(93j:14071)

Steklov Mathematical Institute, Gubkina 8, Moscow, Russia, and Laboratoire Émile PiCard, Université Paul Sabatier, 118 route de Narbonne, 31062 Toulouse Cedex 9, France

E-mail address: orevkov@math.ups-tlse.fr

Received 31/AUG/2006

Translated by THE AUTHOR 